\title{
Quality Assurance FY 1995 Site Support Program Plan WBS 6.7.2.5
}

Prepared for the U.S. Department of Energy

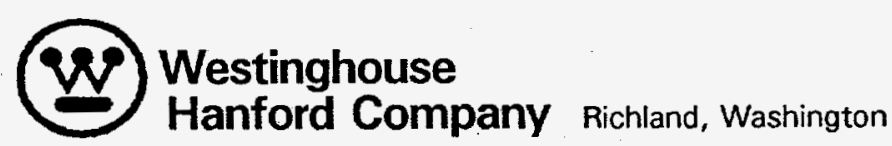

Hanford Operations and Engineering Contractor for the

U.S. Department of Energy under Contract DE-AC06-87RL10930 


\section{Quality Assurance FY 1995 Site Support Program Plan WBS 6.7.2.5}

L. D. Dell

Date Published

September 1994

Prepared for the U.S. Department of Energy

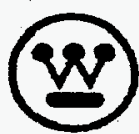




\section{LEGAL DISCLAIMER}

This report was prepared as an account of work sponsored by an agency of the United States Government. Neither the United States Government nor any agency thereof, nor any of their employees, nor any of their contractors, subcontractors or their employees, makes any warranty, express or implied, or assumes any legal liability or responsibility for the accuracy, completeness, or any third party's use or the results of such use of any information, apparatus, product, or process disclosed, or represents that its use would not infringe privately owned rights. Reference herein to any specific commercial product, process, or service by trade name, irademark, manufacturer, or otherwise, does not necessarily constitute or imply its endorsement, fecommendation, or favoring by the United States Government or any agency thereof or its contractors ar subcontractors. The views and opinions of authors expressed herein do not necessarily state or reflect those of the United States Government or any agency thereot.

This report has been reproduced from the best available copy. Available in paper copy and microfiche.

Available to the U.S. Department of Energy

and its contractors from

Olfice of Scientilic and Technical Information

P.O. Box 62

Oak Ridge, TN 37831

(615) $576-8401$

Available to the public from the U.S. Department of Commerce National Technical Information Service

5285 Port Royal Road

Springtield, VA 22161

(703) $487-4650$

Printed in the United States of America 


\section{DISCLAIMER}

Portions of this document may be illegible in electronic image products. Images are produced from the best available original document. 


\section{RELEASE AUTHORIZATION}

\begin{tabular}{ll} 
Document Number: & WHC-SP-1108 \\
\hline Document Title: & Qual ity Assurance Fiscal Year 1995 Site Support
\end{tabular}

\begin{aligned} & Program Plan WBS 6.7.2.5 \\ \hline Release Date: & $9 / 19 / 94 \\$\hline & $* * * * * * * * * * * *\end{aligned}$

This document was reviewed following the procedures described in WHC-CM-3-4 and is:

APPROVED FOR PUBLIC RELEASE

* * ************

WHC Information Release Administration Specialist:

(-hinativistellexplam

C. Will ingham

$9 / 19 / 94$

(Signature)

(Date) 
WHO -S P-1108

Site Support Program Plan Approval Sheet

QUALITY ASSURANCE - WBS \#6.7.2.5

Assistant Manager-Contracting Officer's Representative
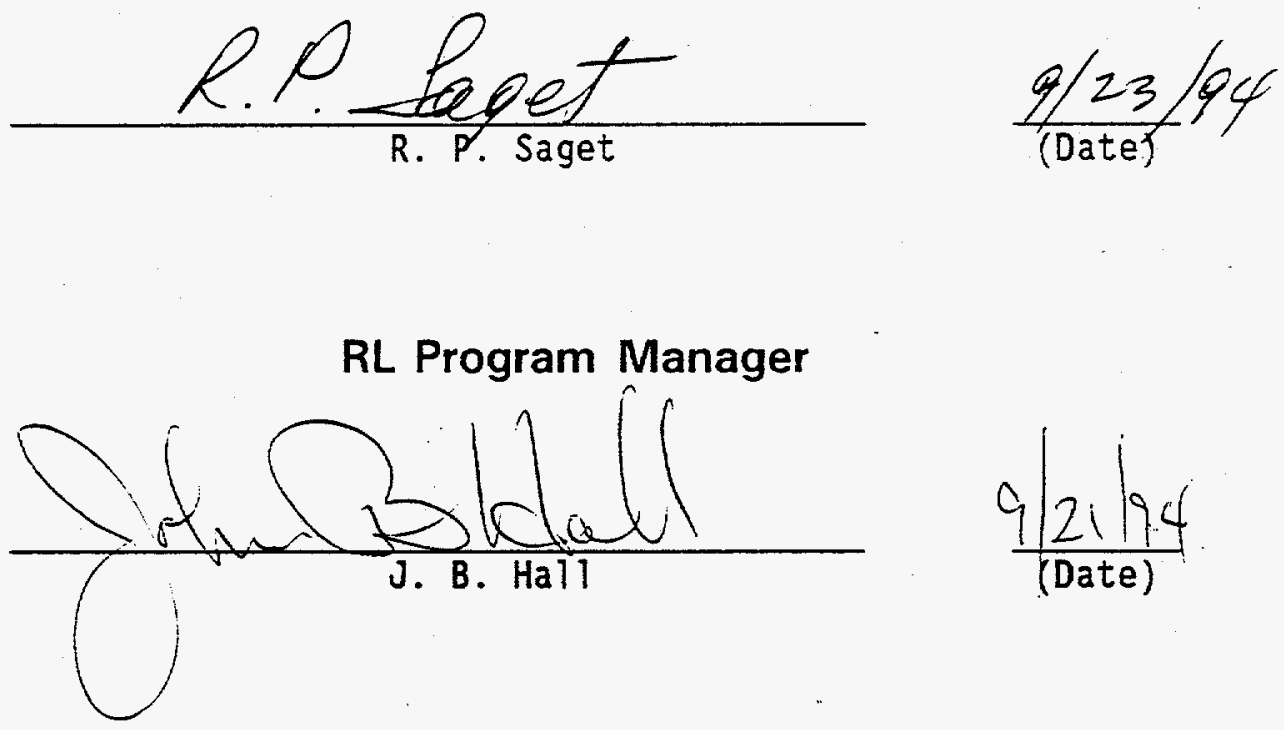

WHC Director

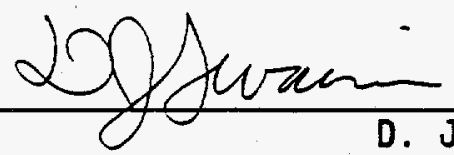

$\frac{9 / 23 / 54 /}{\text { (Date) }}$

WHC Program Manager

P. R. Praetorius

$$
\frac{9 / 20 / 94}{\text { (Date) }}
$$


WHC-SP-1108

Site Support Program Plan Approval Sheet

WBS 6.7.2.5 Quality Assurance

Quality Assurance Program Monitor

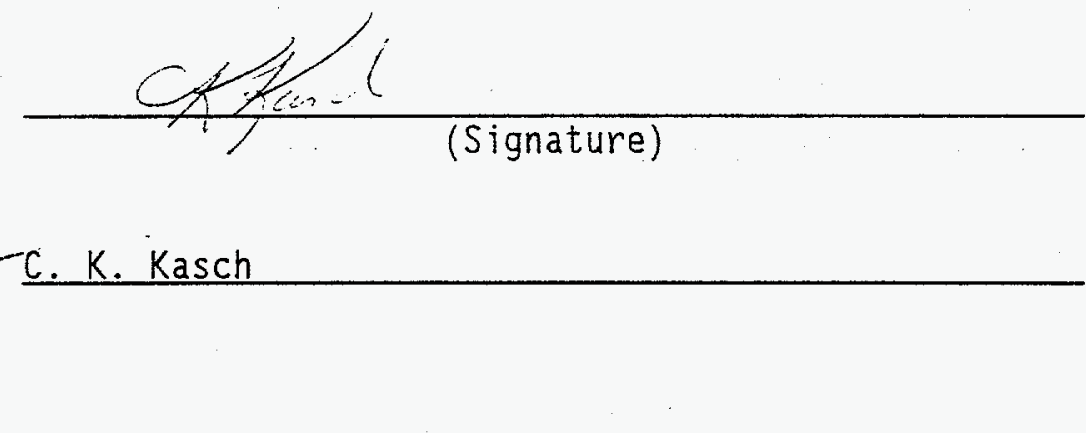

$$
\frac{\text { g/eifys }}{\text { (Date) }}
$$

$\frac{k m v}{9 / 20 / 94}$

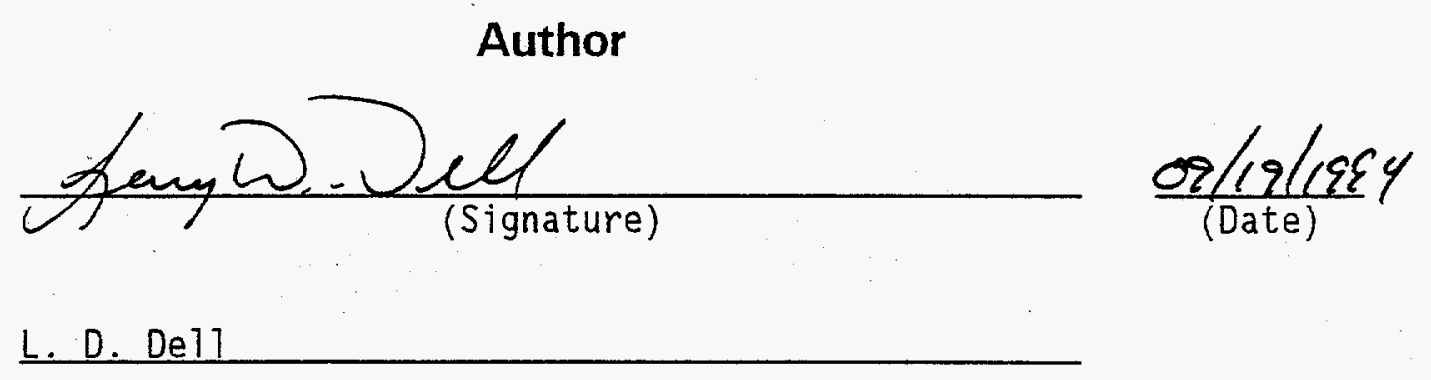

SSPPAPR3.SHT 


\section{Quality Assurance Fiscal Year 1995 Site Support Program Plan WBS 6.7.2.5}

Section 1. Overview

Section 2. Baseline

2.A.1 WBS \& Responsibility Assignment Matrix

2.A.2 Description of Activities

2.B.1 Milestone List

$9-10$

2.C.1 Cost Baseline by Program Element

$11-14$

2.C.1.1 Cost Baseline by Funding Source

$15-16$

2.C.2 Cost Basis

2.C.3 FTE Forecasts

Section 3. Execution Year

3.A WBS Dictionary

3.B Milestone Decription Sheets 
CAP
LEVEL
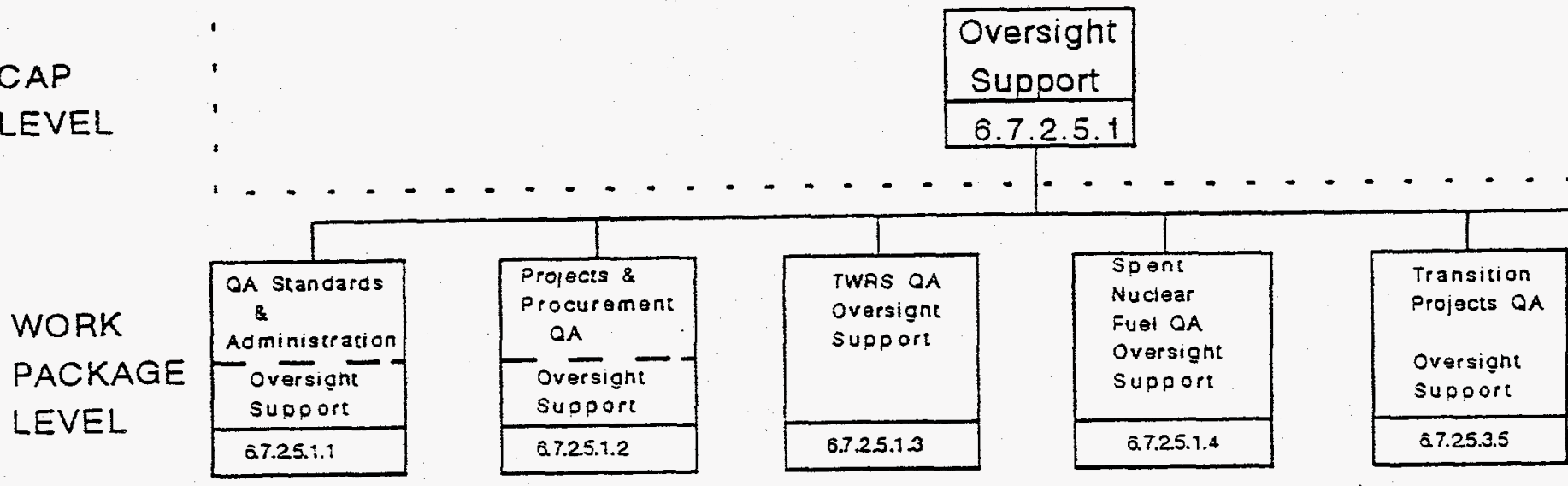

\begin{tabular}{|c|c|c|c|c|c|}
\hline $\begin{array}{l}\text { TASK } \\
\text { LEVEL }\end{array}$ & $\begin{array}{l}\text { a7.25.1.1.01 } \\
\text { OA Stancards \& } \\
\text { Administration } \\
\text { 67.25.11.02 } \\
\text { WHC Self } \\
\text { Assessment } \\
\text { 67.25.1.1.08 } \\
\text { Software } \\
\text { Ovarsignt } \\
\text { \& Support } \\
\text { a.25.1.1.04 } \\
\text { Document } \\
\text { Control \& } \\
\text { Fecords } \\
\text { Management } \\
\text { a.7.25.1.1.05 } \\
\text { OA Self } \\
\text { Assessment } \\
\text { Goordination }\end{array}$ & 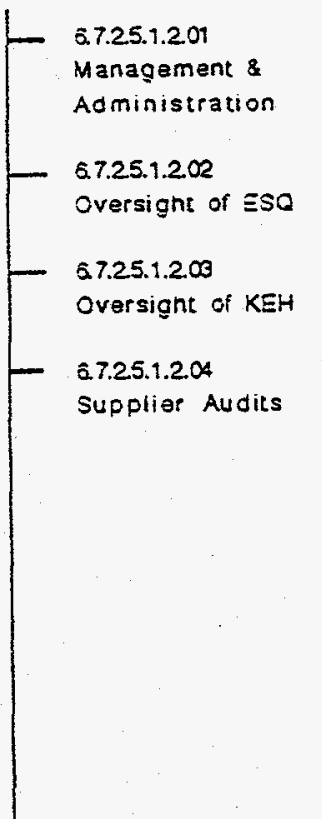 & $\begin{array}{l}\text { 5.25.25.13.01 } \\
\text { TWRS Audit, } \\
\text { Task } \\
\text { Taam. } \\
\text { Assessments }\end{array}$ & $\begin{array}{l}\text { 67.25.1.4.01 } \\
\text { Spent Nuclear } \\
\text { Fuei } \\
\text { Audit. Task } \\
\text { Team. } \\
\text { Assessments }\end{array}$ & $\begin{array}{l}\text { 6.7.25.1.5.01 } \\
\text { Transition } \\
\text { Projects } \\
\text { Audit. Task } \\
\text { Team, } \\
\text { Assessments }\end{array}$ \\
\hline Exempt & 6.25 & 5.50 & 1.50 & .50 & 1.50 \\
\hline Non-Exempt & 0.25 & 1.00 & & & \\
\hline FTE Totals & 0.50 & 6.50 & 1.50 & .50 & 1.50 \\
\hline
\end{tabular}




\section{QUALITY ASSURAN WORK BREAKDOWN ST}

PROGRAM

LEVEL

CAP

LEVEL
Quality Assurance Pr

WBS 6.7.2.5
WORK

PACKAGE

LEVEL

TASK

LEVEL

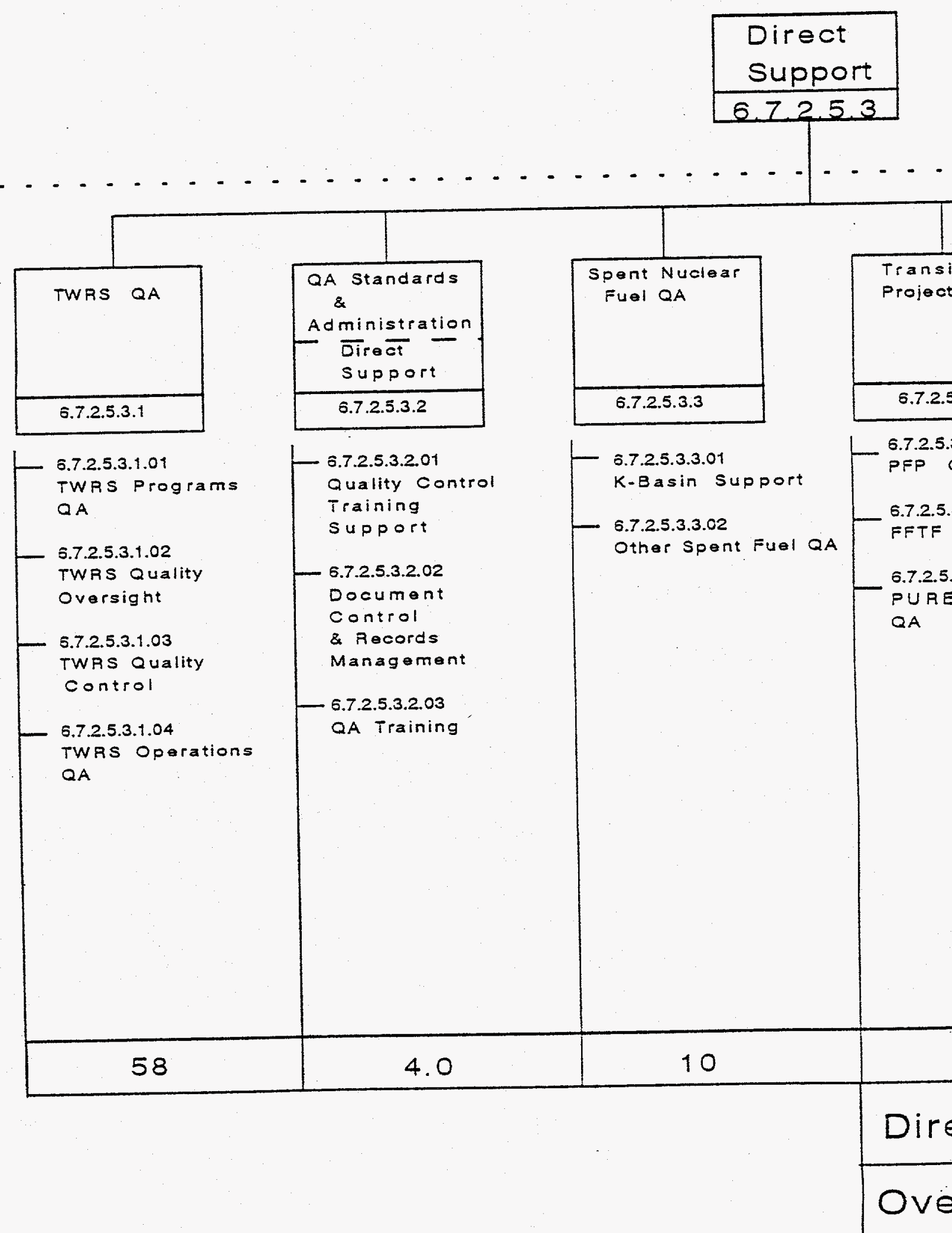




\section{SE \\ RUCTURE}

\section{ogram}

on

QA

.

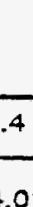

4.01

A

4.02

QA

4.03

/B-Plant

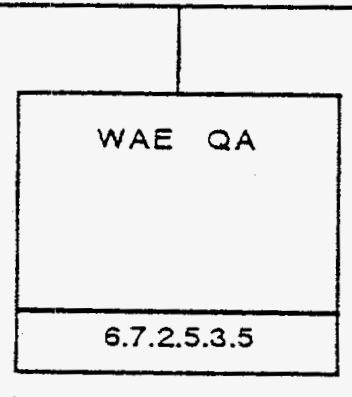

6.7.2.5.3.5.01 Analytical Services QA

6.7.2.5.3.5.02 Engineering and Environmental $Q A$

6.7.2.5.3.5.03 Waste Operations QA

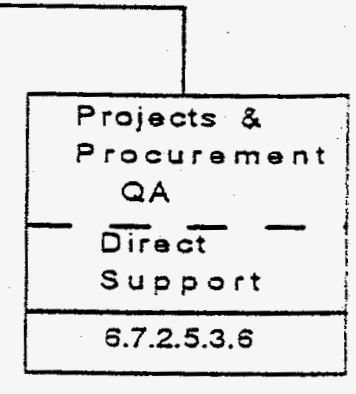

6.7.2.5.3.6.01

Fabrication

Shop

QA

6.7.2.5.3.6.02

Supplier

Evaluation

6.7.2.5.3.5.03

QA Support

for

stores

6.7.2.5.3.6.04

HEPA Filter

$T$ esting

6.7 .2 .5 .3 .6 .05

Feceiving

Inspection

\section{t}

.

.

II Total

204.0

172.5

19.5

37

\begin{tabular}{|l|l|}
\hline Total & 172.5 \\
\hline 11 Total & 204.0 \\
\hline
\end{tabular}




\begin{tabular}{|c|c|c|}
\hline 1.A Vision/Mission & $\begin{array}{c}\text { Westinghouse Hanford Company } \\
\text { Quality Assurance } \\
6.7 .2 .5\end{array}$ & $\begin{array}{c}\text { FY 1995 } \\
\text { Site Support Program Plan } \\
\text { Date Prepared: 09/16/94 }\end{array}$ \\
\hline
\end{tabular}

The mission of the quality Assurance organization, is to:

- Provide leadership in establishing and interpreting company quality assurance program.

- Support line organizations in implementing a quality assurance program that is oriented towards preventing problems and is capable of confirming the attainment of quality.

The Quality Assurance function verifies that the appropriate quality assurance programs and controls are applied to activities that affect quality including:

- Waste management;

- Environmental activities (restoration, remediation, and monitoring);

- Implementation of environmental, state, local, and federal regulations;

- Tri-Party Agreement activities;

- Facility operation and deactivation/transition to shutdown; and

- New facility construction and operation. 


\begin{tabular}{|c|c|c|}
\hline $\begin{array}{c}\text { Situational Analysis } \\
\text { 1.B.1 Internal Assessment } \\
\text { Summary }\end{array}$ & $\begin{array}{c}\text { Westinghouse Hanford Company } \\
\text { Qual ity Assurance } \\
6.7 .2 .5\end{array}$ & $\begin{array}{c}\text { FY 1995 } \\
\text { Site Support Program Plan } \\
\text { Date Prepared: 09/16/94 }\end{array}$ \\
\hline
\end{tabular}

Quality Assurance products and services are organized into the following areas:

- Oversight Support activities include: (a) management and administration of the Quality Assurance oversight functions; (b) coordinating Westinghouse Hanford Company self-assessment activities, including maintenance of plans, preparation of reports, preparation/maintenance of governing policy/procedures; (c) providing oversight of Westinghouse Hanford Company software development, maintenance, and operations, and Westinghouse Hanford Company document control, records management, and other support service activities, including surveillance planning, scheduling, reporting, follow-up, and problem resolution; (d) performing supplier audits; and (g) coordination of the Quality Assurance self-assessment activities.

- Overhead Support activities include: (a) management, supervision, and administration of the Quality Assurance program; (b) maintenance and administration of Westinghouse Hanford Company Quality Assurance policies, procedures, and manuals; (c) establishing, coordinating, and conducting training for Quality Assurance and Quality Control personnel; (d) management and administration of services provided through the Quality Assurance Basic Ordering Agreement; (e) development of the Quality Assurance Site Support Program Plan; and (f) oversight of the Environmental, Safety, and quality Assurance program activities.

- Direct Support activities include: (a) providing quality control, quality engineering, and independent oversight functions necessary to support Tank Waste Remediation System; Spent Nuclear Fuel; Transition Projects; Waste, Analytical, and Environmental facilities, and procurement activities; (b) training and administration of the Quality Control inspector qualification and certification program; (c) quality engineering support to software, document control, and records management functions; (d) Total Quality facilitation and training services; (e) providing independent quality control, quality engineering, and oversight functions necessary to support site-wide activities related to engineering, procurement, construction, fabrication, and operating the High Efficiency Particulate Air filter test facility.

Significant Quality Assurance activities are those activities that are directed at establishing the quality requirements for the performance of activities and verifying that the activities have been implemented in conformance with these requirements. This results in the highest value added by assuring that work is conducted in accordance with customer requirements. 


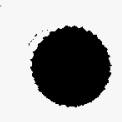

Situational Analysis

1.B.1 Internal Assessment Summary
Westinghouse Hanford Company

quality Assurance

6.7.2.5
FY 1995

Site Support Program Plan

Date Prepared: 09/16/94

\section{Internal Assessment Summary (cont inued)}

The overall cost of the Quality Assurance program for FY 1995 is estimated to be $\$ 19,649,000$, while the Quality Assurance program elements will require funding at the following levels:

- $\quad$ Oversight Support....\$1,940,000

- $\quad$ Overhead Support.... \$3,100,000

- Direct Support.....\$14,600,000

The cost estimates reflect a change in the method of cost allocation from Fy 1994 . The majority of costs associated with supporting the Direct Programs will be funded by Direct Programs in FY 1995, instead of overhead/oversight pools. This has resulted in a reduction of the overhead rate to approximately $25 \%$ from $44 \%$. The varied types and scope of products, services, and customers make it impractical to define unit costs at this time. However, the Quality Assurance organization will attempt to standardize cost accounting practices during FY 1995 in order to better define unit costs for FY 1996 planning purposes. History and analogy were used to develop the cost estimate.

Significant changes are anticipated in the volume and costs associated with Quality Assurance products and services. This is due to the implementation of an Environmental Restoration Contract by the Department of Energy (15 full-time equivalents) and productivity improvements ( 7 full-time equivalents). 0ther scope increases (5 full-time equivalents) will partially off set the previous reductions in work scope for a net reduction in full-time equivalents of 17 in FY 1995. There may be some impact resulting from redirecting quality assurance requirements to the line organizations. However, it is not possible to determine the impact at this time. Current products and services appear to be acceptable; products and services have been provided on schedule and under budget. Preliminary results of customer feedback regarding the effectiveness of oversight support indicates a rating of "very effective." Political processes and legislative and regulatory actions provide opportunity for changes in the types or nature of products and services. 


\begin{tabular}{|c|c|c|}
\hline $\begin{array}{c}\text { Situational Analysis } \\
\text { 1.B.2 External Assessiment } \\
\text { Summary }\end{array}$ & $\begin{array}{c}\text { Westinghouse Hanford Company } \\
\text { Quality Assurance } \\
6.7 .2 .5\end{array}$ & $\begin{array}{c}\text { Fy 1995 } \\
\text { Site Support Program Plan } \\
\text { Date Prepared: 09/16/94 }\end{array}$ \\
\hline
\end{tabular}

Quality Assurance investigation of the support required by the Direct Programs has not identified any new requirements. Products and services will be similar in volume and scope to those provided in previous years and will not require significant changes to current Quality Assurance staff capability other than previously mentioned.

Periodic assessment of the adequacy of products and services with the Department of Energy, Richland Operations office will provide adequate opportunity for Quality Assurance to adjust and/or focus support activities to customer needs.

Political processes and legislative and regulatory actions are external drivers which may result in changes in the types or nature of Quality Assurance products and services. 


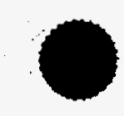

\section{C Goals and Objectives}

Westinghouse Hanford Company Quality Assurance 6.7 .2 .5
FY 1995

Site Support Program Plan Date Prepared: 09/16/94

Quality Assurance management's goal is to staff and manage an organization that is fully supportive of the Quality Assurance mission and WHC policies.

It is also our goal to provide leadership in establishing quality assurance policy and ensure that the Quality Assurance Manual (WHC-CM-4-2) fully complies with applicable quality assurance requirements. This goal will be satisfied by accomplishment of the following objectives:

- Issue Quality Assurance Program Description and Implementation Plan for Rule 10CFR830.120 and

- Issue instructions for preparing Quality Assurance Program Plans (QAPPs) based upon Rule 10CFR830.120.

Another goal is to interpret quality assurance program requirements and to develop practical methods for 1 ine implementation of these requirements. This goal will be satisfied by accomplishment of the following objective:

- Maintain Westinghouse Hanford Company quality assurance program manuals to implement the quality assurance policies.

It is also our goal to perform surveillances, inspections, assessments, and integrated management team and supplier audits as necessary to provide independent evaluation of the level of quality performance achieved. This goal will be satisfied by accomplishment of the following objectives:

- Develop and issue semi-annual schedule for chartered oversight activities of Projects and Procurement Oversight Support including oversight of Emergency, Safety, \& Quality Services, oversight of ICF Kaiser, and supplier audits;

- Performance updates for activities performed the previous period; and

- Performance of surveillances against FY 1995 schedules.

Another goal is to execute a strong quality assurance program to help the efficiency and effectiveness of all programs in their use of resources. This goal will be satisfied by accomplishment of the following objectives:

- Control nonconforming items and pursue actions to correct adverse conditions and prevent recurrence and

- Provide quality engineering and quality control services. 


\begin{tabular}{|c|c|c|}
\hline 1.C Goals and objectives & $\begin{array}{c}\text { Westinghouse Hanford Company } \\
\text { Qual ity Assurance } \\
6.7 .2 .5\end{array}$ & $\begin{array}{c}\text { Fy 1995 } \\
\text { Site Support Program Plan } \\
\text { Date Prepared: 09/16/94 }\end{array}$ \\
\hline
\end{tabular}

\section{Goals and 0bjectives (continued)}

It is also our goal to perform and coordinate self-assessments to achieve continuous improvement. This goal will be satisfied by accomplishment of the following objectives:

- Issue the Westinghouse Hanford Company Self-Assessment Program Implementation Plan update;

- Issue the mid-year Westinghouse Hanford Company Self-Assessment Report; and

- Issue the Annual Westinghouse Hanford Company Self-Assessment Report.

Another goal is to manage the quality Assurance program to accomplish quality assurance support services in accordance with established budgets, schedules and technical baselines. This goal will be satisfied by accomplishment of the following objectives:

- Periodically monitor scope, schedule, and costs of services against Fy 1995 Quality Assurance Site Support Program Plan and

- Issue the FY 1996 Quality Assurance Site Support Program Plan. 
Westinghouse Hanford Company Quality Assurance 6.7 .2 .5

\section{FY 1995}

Site Support Program Plan Date Prepared: 09/16/94

Quality Assurance has established basic strategies to achieve the more significant goals and objectives. The strategies for the key goals and objectives are as follows:

Quality Assurance will verify that quality assurance policy and the Quality Assurance Manual (WHC-CM-4-2) fully comply with applicable quality assurance requirements established by Rule 10CFR830.120. This will be accomplished by performing a comprehensive review of the Rule to identify any omissions in the current program. Action plans will be developed to revise the Quality Assurance Program and implementing procedures to address identified deficiencies. Milestones have been established to track development of plans and schedules for updating the program. Planning activities will be completed during the first quarter of FY 1995 and will establish the schedule for updating the program. The activities will be accomplished by the Quality Assurance Standards and Administration (QAS\&S) group, with considerable involvement with project organizations, other quality assurance organizations, and the Department of Energy, Richland Operations Office. These activities will be funded from the Overhead Support budget. Related goals and objectives have been established for training personnel in, and maintenance of, the Quality Assurance Program.

Quality Assurance will perform surveillances, inspections, assessments, and integrated management team and supplier audits to evaluate the level of quality performance achieved. This will be accomplished by developing a rigorous schedule of evaluation activities; preparing evaluation plans, procedures and criteria; implementing the plans and procedures; reporting the results of the evaluation activities; and performing follow up activities to confirm the resolution of any problems identified. Milestones have been established to track development and issue of the surveillance schedules. The schedules will be issued semi-annually by Quality Assurance oversight organizations funded from the oversight Support budget.

Quality Assurance organizations will perform self-assessment. This goal will be accomplished by developing a plan to identify the areas and/or activities to be assessed, the performance objectives and criteria to be used during the assessment, and the schedule for performing the assessments for the quality assurance organization. The results of the assessments will be reported and corrective action will be established to effect quality assurance program enhancements.

Quality Assurance will also coordinate the Westinghouse Hanford Company self-assessment effort. Coordination of assessment activities has been assigned to the QAS\&A organization and will be funded from the 0versight Support budget. In addition, some self-assessment activities will be funded from the Direct Programs.

Milestones have been established to track development of the WHC self assessment implementation plan update and issuance of mid-year and annual reports for QA and WHC. 


\begin{tabular}{|c|c|c|}
\hline 1.D Strategies & Westinghouse Hanford Company \\
(cont.) & $\begin{array}{c}\text { Fual ity Assurance } 1995 \\
6.7 .2 .5\end{array}$ & $\begin{array}{c}\text { Site Support Program Plan } \\
\text { Date Prepared: 09/16/94 }\end{array}$ \\
\hline
\end{tabular}

The Quality Assurance Program will be managed to accomplish support services in accordance with established budgets and schedules. This will be accomplished by developing a comprehensive Site Support Program Plan to identify the costs of performing quality assurance services and then periodically monitoring the performance of the program to determine whether activities have been performed in accordance with established budgets and within schedules. Comparison of actual cost and schedule performance of services versus the budget and planned schedule will be reported using the monthly Site Management System reporting process and will be used as a basis for taking corrective action. Development of the FY 1996 Quality Assurance Site Support Program Plan has been assigned to the QAS\&A group and will be funded from the Overhead Support budget. 


\begin{tabular}{|c|c|c|}
\hline 1.E Assumptions & $\begin{array}{c}\text { Westinghouse Hanford Company } \\
\text { Quality Assurance } \\
6.7 .2 .5\end{array}$ & $\begin{array}{c}\text { FY } 1995 \\
\text { Site Support Program Plan } \\
\text { Date Prepared: 09/16/94 }\end{array}$ \\
\hline
\end{tabular}

Quality Assurance goals, objectives, and strategies have been developed on the following assumptions:

- The majority of project aligned activities, including those activities funded through indirect pools in FY 1994, will be direct funded.

- Funding levels required to perform Quality Assurance activities will be sufficient to administer a high quality Quality Assurance program. 


\begin{tabular}{|c|c|c|}
\hline 1.F Issues \& Constraints & $\begin{array}{c}\text { Westinghouse Hanford Company } \\
\text { Quality Assurance } \\
6.7 .2 .5\end{array}$ & $\begin{array}{c}\text { Fy 1995 } \\
\text { Dite Support Program Plan Prepared: 09/16/94 }\end{array}$ \\
\hline
\end{tabular}

Results from the accomplishment of some of the Quality Assurance goals and objectives will identify the need for some line organizations to develop or revise and implement their quality Assurance Program-related plans and procedures and to provide their employees with appropriate training or orientation. This Site Support Program Plan does not cover these line organization efforts and their associated costs. 


\section{G Performance Measures}

Westinghouse Hanford Company

Quality Assurance

6.7.2.5
FY 1995

Site Support Program Plan

Date Prepared: 09/16/94

A number of the Quality Assurance goals and objectives are directly linked to a number of performance measures used to determine Award Fee Performance. These include:

- Providing proactive and effective leadership and management for the development and execution of programs that assure operations and activities are environmentally sound and provide protection of the health and safety of workers and the public, and that comply with the applicable requirements in the area of quality as surance.

- Providing leadership in process improvement and innovation through the integration of a total quality approach into all facets of activities.

- Providing effective and efficient management of the budgetary resources in executing current $F i s c a l$ Year Work Plans, Site Support Program Plans, and responding to changing circumstances with appropriate consideration of long range plans and objectives.

- Assuring quality requirements are met.

In addition, Quality Assurance performance measures include:

- Customer satisfaction as measured from feedback surveys, regular DOE-RL interface meetings, and the Site Management System process.

- Completion of Stretch Goals as a means of improving overall award fee rating.

- Compliance with milestone schedules. 


\begin{tabular}{|c|c|c|c|c|c|}
\hline \multicolumn{2}{|c|}{$\begin{array}{c}2 . A .1 \\
\text { WBS \& Responsibility } \\
\text { Assignment } \\
\text { Matrix }\end{array}$} & \multicolumn{2}{|r|}{$\begin{array}{c}\text { Westinghouse Hanford Company } \\
\text { Qual ity Assurance } \\
6.7 .2 .5\end{array}$} & \multicolumn{2}{|c|}{$\begin{array}{l}\text { FY } 1995 \\
\text { Site Support Program Plan } \\
\text { Date Prepared: } 09 / 16 / 94\end{array}$} \\
\hline $\begin{array}{l}\text { Activity } \\
\text { No. }\end{array}$ & $\begin{array}{c}\text { Cost } \\
\text { Account }\end{array}$ & $\begin{array}{l}\text { WBS Work } \\
\text { Package }\end{array}$ & Title & $\begin{array}{l}\text { Responsible } \\
\text { Manager }\end{array}$ & $\begin{array}{l}\text { Responsible } \\
\text { Organizat ion }\end{array}$ \\
\hline 6.7 .2 .5 & & & QA Program Support & A.J Fisher & 38000 \\
\hline 6.7 .2 .5 .1 & IMDM05 & 6.7 .2 .5 .1 & QA Oversight Support & As $\mathrm{F}$ isher & 38000 \\
\hline 6.7 .2 .5 .1 .1 .01 & 1MDM05 & & $\begin{array}{l}\text { QA Standards \& Administration - Oversight } \\
\text { Support }\end{array}$ & HE ReW & 38300 \\
\hline 6.7 .2 .5 .1 .1 .02 & 1MDM05 & & WHC Self-Assessment & HE Rew & 38300 \\
\hline 6.7 .2 .5 .1 .1 .03 & 1MDM05 & & Software Oversight and Support & HE Rew & 38300 \\
\hline 6.7 .2 .5 .1 .1 .04 & 1MDM05 & & Document Control \& Records Management & HE Rew & 38300 \\
\hline \multirow[t]{2}{*}{6.7 .2 .5 .1 .1 .05} & 1MDM05 & & QA Self-Assessment Coordination & HE Rew & 38300 \\
\hline & 1MDM05 & 6.7 .2 .5 .1 .2 & $\begin{array}{l}\text { QA Projects \& Procurement - Oversight } \\
\text { Support }\end{array}$ & JA Peltier & 38900 \\
\hline $6.7 .2 .5 \cdot 1 \cdot 2.01$ & IMDM05 & & Oversight of ESQ & JA Peltier & 38900 \\
\hline 6.7 .2 .5 .1 .2 .02 & 1MDM05 & & Projects \& Procurement Quality Oversight & JA Peltier & 38900 \\
\hline $6.7 .2 .5 \cdot 1.2 .03$ & 1MDM05 & & Oversight of KEH & JA Peltier & 38900 \\
\hline \multirow[t]{2}{*}{6.7 .2 .5 .1 .2 .04} & 1MDM05 & & Supplier Audits & JA Peltier & 38900 \\
\hline & 1MDM05 & $6.7 .2 .5 \cdot 1.3$ & $\begin{array}{l}\text { Tank Waste Remediation System QA Oversight } \\
\text { Support }\end{array}$ & $J$ Weber & 38200 \\
\hline \multirow[t]{2}{*}{6.7 .2 .5 .1 .3 .01} & IMDM05 & & $\begin{array}{l}\text { Tank Waste Remediation System Audit, Task } \\
\text { Team, Assessments }\end{array}$ & $J$ Weber & 38200 \\
\hline & 1MDM05 & 6.7 .2 .5 .1 .4 & Spent Nuclear Fuel QA Oversight Support & DW Smith & 38400 \\
\hline
\end{tabular}




\begin{tabular}{|c|c|c|c|c|c|}
\hline \multicolumn{2}{|c|}{$\begin{array}{c}2 . A .1 \\
\text { WBS \& Responsibility } \\
\text { Assignment } \\
\text { Matrix }\end{array}$} & \multicolumn{2}{|r|}{$\begin{array}{c}\text { Westinghouse Hanford Company } \\
\text { Quality Assurance } \\
6.7 .2 .5\end{array}$} & \multicolumn{2}{|c|}{$\begin{array}{l}\text { FY } 1995 \\
\text { Site Support Program Plan } \\
\text { Date Prepared: } 09 / 16 / 94\end{array}$} \\
\hline $\begin{array}{c}\text { Activity } \\
\text { No. }\end{array}$ & $\begin{array}{c}\text { Cost } \\
\text { Account }\end{array}$ & $\begin{array}{l}\text { WBS Work } \\
\text { Package }\end{array}$ & Title & $\begin{array}{l}\text { Respons ible } \\
\text { Manager }\end{array}$ & $\begin{array}{l}\text { Responsible } \\
\text { Organization }\end{array}$ \\
\hline \multirow[t]{2}{*}{6.7 .2 .5 .1 .4 .01} & $1 M O M 05$ & & $\begin{array}{l}\text { Spent Nuclear Fuel Audit, Task Team, } \\
\text { Assessinents }\end{array}$ & DH Smith & 38400 \\
\hline & 1MDM05 & 6.7 .2 .5 .1 .5 & Transition Projects $Q A$ Oversight Support & JN Nansen & 38500 \\
\hline \multirow[t]{2}{*}{6.7 .2 .5 .1 .5 .01} & $1 M D M 05$ & & $\begin{array}{l}\text { Transition Projects Audit, Task Team, } \\
\text { Assessments }\end{array}$ & JN Nansen & 38500 \\
\hline & 1MDM05 & 6.7 .2 .5 .1 .6 & $\begin{array}{l}\text { Waste, Environmental, \& Analytical QA } \\
\text { Oversight Support }\end{array}$ & PR Praetorius & 38600 \\
\hline 6.7 .2 .5 .1 .6 .01 & 1MDM05 & & $\begin{array}{l}\text { Waste, Environmental, \& Analytical QA Audit, } \\
\text { Task Team, Assessments }\end{array}$ & PR Praetorius & 38600 \\
\hline 6.7 .2 .5 .1 .6 .02 & 1MDM05 & & Suspect/Counterfeit Parts & PR Praetorius & 38600 \\
\hline \multirow[t]{2}{*}{6.7 .2 .5 .2} & $1 \mathrm{~J} 3800$ & 6.7 .2 .5 .2 & QA Overhead Support & AJ Fisher & 38000 \\
\hline & $1 J 3800$ & 6.7 .2 .5 .2 .1 & QA Program Overhead & AJ fisher & 38000 \\
\hline \multirow[t]{2}{*}{6.7 .2 .5 .2 .1 .01} & $1 \mathrm{~J} 3800$ & & $\begin{array}{l}\text { QA Program Management, Administration \& } \\
\text { Operational Expenses }\end{array}$ & AJ fisher & 38000 \\
\hline & $1 \mathrm{~J} 3800$ & 6.7 .2 .5 .2 .2 & $\begin{array}{l}\text { QA Standards \& Administration Overhead } \\
\text { Support }\end{array}$ & HE ReW & 38300 \\
\hline 6.7 .2 .5 .2 .2 .01 & $1 \mathrm{~J} 3800$ & & $\begin{array}{l}\text { QA Standards \& Administration - Management \& } \\
\text { Administration }\end{array}$ & HE Rew & 38300 \\
\hline 6.7 .2 .5 .2 .2 .02 & $1 \mathrm{~J} 3800$ & & Program Planning & HE Rew & 38300 \\
\hline 6.7 .2 .5 .2 .2 .03 & $1 \mathrm{~J} 3800$ & & Site Management System Reporting & HE Rew & 38300 \\
\hline 6.7 .2 .5 .2 .2 .04 & $1 \mathrm{~J} 3800$ & & QA Manual Maintenance & HE Rew & 38300 \\
\hline
\end{tabular}




\begin{tabular}{|c|c|c|c|c|c|}
\hline \multicolumn{2}{|c|}{$\begin{array}{c}2 . \text { A.1 } \\
\text { WBS Responsibility } \\
\text { Assignment } \\
\text { Matrix }\end{array}$} & \multicolumn{2}{|r|}{$\begin{array}{c}\text { Westinghouse Hanford Company } \\
\text { Qual ity Assurance } \\
6.7 .2 .5\end{array}$} & \multicolumn{2}{|c|}{$\begin{array}{l}\text { FY } 1995 \\
\text { Site Support Program Plan } \\
\text { Date Prepared: 09/16/94 }\end{array}$} \\
\hline $\begin{array}{l}\text { Activity } \\
\text { No. }\end{array}$ & $\begin{array}{l}\text { Cost } \\
\text { Account }\end{array}$ & $\begin{array}{r}\text { WBS Work } \\
\text { Package } \\
\end{array}$ & Title & $\begin{array}{l}\text { Responsible } \\
\text { Manager }\end{array}$ & $\begin{array}{l}\text { Responsible } \\
\text { Organization }\end{array}$ \\
\hline \multirow[t]{2}{*}{6.7 .2 .5 .2 .2 .05} & $1 J 3800$ & & RL Interface & HE ReW & 38300 \\
\hline & $1 \mathrm{~J} 3800$ & 6.7 .2 .5 .2 .3 & Projects \& Procurement Overhead Functions & JA Peltier & 38900 \\
\hline $6.7 .2 \cdot 5 \cdot 2 \cdot 3.01$ & $1 J 3800$ & & $\begin{array}{l}\text { Projects \& Procurement Management \& } \\
\text { Administration }\end{array}$ & JA Peltier & 38900 \\
\hline $6.7 \cdot 2 \cdot 5 \cdot 2.3 .02$ & $1 J 3800$ & & QA Support of ESQ & JA Peltier & 38900 \\
\hline \multirow[t]{2}{*}{6.7 .2 .5 .3} & Direct Caps & 6.7 .2 .5 .3 & QA Direct Support & JA Fisher & 38000 \\
\hline & & 6.7 .2 .5 .3 .1 & TWRS QA & I weber & 38200 \\
\hline 6.7 .2 .5 .3 .1 .01 & & & TWRS Programs QA & $J$ weber & 38200 \\
\hline 6.7 .2 .5 .3 .1 .02 & & & TWRS Quality Oversight & J Weber & 38200 \\
\hline 6.7 .2 .5 .3 .1 .03 & & & TWRS Quality Control & J weber & 38200 \\
\hline \multirow[t]{2}{*}{6.7 .2 .5 .3 .1 .04} & & & TWRS Operations QA & \lrcorner weber & 38200 \\
\hline & & 6.7 .2 .5 .3 .2 & QA Standards \& Admin. Direct Support & HE ReW & 38300 \\
\hline 6.7 .2 .5 .3 .2 .01 & & & Quality Control Training Support & HE ReW & 38300 \\
\hline 6.7 .2 .5 .3 .2 .02 & & & Document Control \& Records Management & HE Rew & 38300 \\
\hline \multirow[t]{2}{*}{6.7 .2 .5 .3 .2 .03} & & & QA Training & HE ReW & 38300 \\
\hline & & 6.7 .2 .5 .3 .3 & Spent Nuclear Fuel QA & DW Smith & 38400 \\
\hline 6.7 .2 .5 .3 .3 .01 & & & K-Bas in QA Support & DW Smith & 38400 \\
\hline \multirow[t]{2}{*}{6.7 .2 .5 .3 .3 .02} & & & Other Spent Fuel QA Support & DW Smith & 38400 \\
\hline & & 6.7 .2 .5 .3 .4 & Transition Projects QA & JN Nanson & 38500 \\
\hline
\end{tabular}




\begin{tabular}{|c|c|c|c|c|c|}
\hline \multicolumn{2}{|c|}{$\begin{array}{c}2 . A .1 \\
\text { WBS \& Responsibility } \\
\text { Assignment } \\
\text { Matrix }\end{array}$} & \multicolumn{2}{|r|}{$\begin{array}{c}\text { Westinghouse Hanford Company } \\
\text { Quality Assurance } \\
6.7 .2 .5\end{array}$} & \multicolumn{2}{|c|}{$\begin{array}{l}\text { FY } 1995 \\
\text { Site Support Program Plan } \\
\text { Date Prepared: 09/16/94 }\end{array}$} \\
\hline $\begin{array}{c}\text { Activity } \\
\text { No. }\end{array}$ & $\begin{array}{c}\text { Cost } \\
\text { Account }\end{array}$ & $\begin{array}{l}\text { WBS Work } \\
\text { Package } \\
\end{array}$ & Title & $\begin{array}{l}\text { Responsible } \\
\text { Manager }\end{array}$ & $\begin{array}{l}\text { Responsible } \\
\text { Organization }\end{array}$ \\
\hline 6.7 .2 .5 .3 .4 .01 & & & PFP QA & JN Nanson & 38500 \\
\hline 6.7 .2 .5 .3 .4 .02 & & & FFTF QA & JN Nanson & 38500 \\
\hline \multirow[t]{2}{*}{6.7 .2 .5 .3 .4 .03} & & & Purex/B-Plant $Q A$ & JN Nanson & 38500 \\
\hline & & 6.7 .2 .5 .3 .5 & $\begin{array}{l}\text { Waste, Analytical \& Environmental (WAE) } \\
\text { Programs QA }\end{array}$ & PR Praetorius & 38600 \\
\hline 6.7 .2 .5 .3 .5 .01 & & & WAE - Analytical Services QA & PR Praetorius & 38600 \\
\hline 6.7 .2 .5 .3 .5 .02 & & & WAE - Environmental Management Services QA & PR Praetorius & 38600 \\
\hline 6.7 .2 .5 .3 .5 .03 & & & WAE - Waste Operations QA & PR Praetorius & 38600 \\
\hline 6.7 .2 .5 .3 .5 .04 & & & WAE - Environmental QA & PR Praetorius & 38600 \\
\hline \multirow[t]{2}{*}{6.7 .2 .5 .3 .5 .05} & & & WAE - Engineered Applications QA & PR Praetorius & 38600 \\
\hline & & $6.7 \cdot 2 \cdot 5.3 .6$ & Projects \& Procurement $Q A$ & JA Peltier & 38900 \\
\hline 6.7 .2 .5 .3 .6 .01 & . & & Fabrication Shop QA & JA Peltier & 38900 \\
\hline 6.7 .2 .5 .3 .6 .02 & & & Supplier Evaluation & JA Peltier & 38900 \\
\hline 6.7 .2 .5 .3 .6 .03 & & & QA Support for Stores & JA Peltier & 38900 \\
\hline 6.7 .2 .5 .3 .6 .04 & & & HEPA Filter Testing & JA Peltier & 38900 \\
\hline 6.7 .2 .5 .3 .6 .05 & & & Receiving Inspection & JA Peltier & 38900 \\
\hline
\end{tabular}


1

\begin{tabular}{|c|c|c|}
\hline $\begin{array}{l}\text { 2. A.2 } \\
\text { Description } \\
\text { of } \\
\text { Activities }\end{array}$ & \multicolumn{2}{|c|}{$\begin{array}{c}\text { West inghouse Hanford Company } \\
\text { Qual ity Assurance } \\
6.7 \cdot 2.5\end{array}$} \\
\hline Activity No. & Activity Title & Description \\
\hline 6.7 .2 .5 .1 .1 .01 & QAS\&A & $\begin{array}{l}\text { Activities include management, supervision and administration of oversight } \\
\text { activities including but not limited to coordination of the QA and WHC self- } \\
\text { assessments activities and oversight of WHC computer software, document control, } \\
\text { and records management activities. }\end{array}$ \\
\hline 6.7 .2 .5 .1 .1 .02 & $\begin{array}{l}\text { WHC Self- } \\
\text { assessment }\end{array}$ & $\begin{array}{l}\text { The self-assessment is planned, structured, and documented and identifies } \\
\text { performance strengths and weaknesses and, for weaknesses, the root cause and } \\
\text { corrective action. This milestone include the issue of a mid-and-annual status } \\
\text { report on the WHC roll up consolidation of individual units self-assessment } \\
\text { results and a WHC Self-Assessment Program Implementation Plan Update. }\end{array}$ \\
\hline 6.7 .2 .5 .1 .1 .03 & $\begin{array}{l}\text { Software Oversight } \\
\text { and Support }\end{array}$ & $\begin{array}{l}\text { Activities include providing oversight and support of WHC software development, } \\
\text { maintenance, and operation. oversight activities include planning, scheduling, } \\
\text { and performing surveillances, and the follow up of findings and assurance of } \\
\text { problem resolution. }\end{array}$ \\
\hline 6.7 .2 .5 .1 .1 .04 & $\begin{array}{l}\text { Document Control \& } \\
\text { Records Management }\end{array}$ & $\begin{array}{l}\text { Provides oversight of document control and records management activities by } \\
\text { performance of surveillance activities, identification of findings, follow up } \\
\text { and problem resolution. }\end{array}$ \\
\hline 6.7 .2 .5 .1 .1 .05 & $\begin{array}{l}\text { QA Self-assessment } \\
\text { Coordination }\end{array}$ & $\begin{array}{l}\text { The QA self-assessment is planned, structured, documented, and identifies } \\
\text { performance strengths and weaknesses and, for weaknesses, the root cause and } \\
\text { corrective action for the QA Program. This activity includes providing input to } \\
\text { the WHC mid-and-annual status report on self-assessment activities. }\end{array}$ \\
\hline 6.7 .2 .5 .1 .2 .01 & $\begin{array}{l}\text { Projects \& } \\
\text { Procurement QA } \\
\text { Management \& } \\
\text { Administration }\end{array}$ & $\begin{array}{l}\text { Activities include management, supervision and administration of oversight } \\
\text { activities for Projects and Procurement } Q A \text { Oversight Support. }\end{array}$ \\
\hline 6.7 .2 .5 .1 .2 .02 & Oversight of ESQ & $\begin{array}{l}\text { Activities include oversight of WHC ESQ. Oversight activities include planning, } \\
\text { scheduling, and performing surveillances, and the follow up of findings and } \\
\text { assurance of problem resolution. }\end{array}$ \\
\hline
\end{tabular}




\begin{tabular}{|c|c|c|}
\hline $\begin{array}{l}\text { 2. A. } 2 \\
\text { Description } \\
\text { of } \\
\text { Activities }\end{array}$ & \multicolumn{2}{|c|}{$\begin{array}{l}\text { Westinghouse Hanford Company } \\
\text { Qual ity Assurance } \\
6.7 .2 .5\end{array}$} \\
\hline Activity No. & Activity Title & Description \\
\hline 6.7 .2 .5 .1 .2 .03 & Oversight of KEH & $\begin{array}{l}\text { Activities include oversight of ICF Kaiser activities. Oversight activities } \\
\text { include planning, scheduling, and performing surveillances, and the follow up of } \\
\text { findings and assurance of problem resolution. }\end{array}$ \\
\hline 6.7 .2 .5 .1 .2 .04 & Supplier Audits & $\begin{array}{l}\text { Activities include oversight of supplier. Oversight activities include } \\
\text { planning, scheduling, and performing audits, and the follow up of findings and } \\
\text { assurance of problem resolution. }\end{array}$ \\
\hline 6.7 .2 .5 .1 .3 .01 & $\begin{array}{l}\text { Tank Waste } \\
\text { Remediation System } \\
\text { Audit, Task Team, } \\
\text { Assessments }\end{array}$ & $\begin{array}{l}\text { Activities include participation in oversight activities including audits, task } \\
\text { team, and, assessments for Tank Waste Remediation Systems. }\end{array}$ \\
\hline 6.7 .2 .5 .1 .4 .01 & $\begin{array}{l}\text { Spent Nuclear Fuel } \\
\text { Audit, Task Team, } \\
\text { Assessment }\end{array}$ & $\begin{array}{l}\text { Activities include participation in oversight activities including audits, task } \\
\text { team, and, assessments for the Spent Nuclear Fuel project. }\end{array}$ \\
\hline 6.7 .2 .5 .1 .5 .01 & $\begin{array}{l}\text { Transition } \\
\text { Projects Audit, } \\
\text { Task Team, } \\
\text { Assessment } \\
\end{array}$ & $\begin{array}{l}\text { Activities include participation in oversight activities including audits, task } \\
\text { team, and, assessments for Transition Projects. }\end{array}$ \\
\hline 6.7 .2 .5 .1 .6 .01 & $\begin{array}{l}\text { WAE Audit, Task } \\
\text { Team, Assessment }\end{array}$ & $\begin{array}{l}\text { Activities include participation in oversight activities including audits, task } \\
\text { team, and, assessments for Waste, Analytical and Environmental Services. }\end{array}$ \\
\hline 6.7 .2 .5 .1 .6 .02 & $\begin{array}{l}\text { Suspect/ } \\
\text { counterfeit Parts }\end{array}$ & $\begin{array}{l}\text { This activity the preparation of quarterly reports that includes a narrative } \\
\text { summary of activities which have occurred since the last annual report and a } \\
\text { tabulation of the status of each facility-specific action plan to date. } \\
\text { Distribution of an Annual Suspect/Counterfeit Products Report constitutes } \\
\text { completion of an Department of Energy, Richland Operation Office milestone. }\end{array}$ \\
\hline 6.7 .2 .5 .2 .1 .01 & $\begin{array}{l}\text { QA Program } \\
\text { Management \& } \\
\text { Administration }\end{array}$ & $\begin{array}{l}\text { Management and administrative activities which support the operation of the } \\
\text { entire } Q A \text { function and its organizations. Included in this activity are costs } \\
\text { associated with facilities, equipment and materials. }\end{array}$ \\
\hline
\end{tabular}


1

\begin{tabular}{|c|c|c|}
\hline $\begin{array}{l}\text { 2. A.2 } \\
\text { Description } \\
\text { of } \\
\text { Activities }\end{array}$ & \multicolumn{2}{|c|}{$\begin{array}{c}\text { Westinghouse Hanford Company } \\
\text { Qual ity Assurance } \\
6.7 .2 .5\end{array}$} \\
\hline Activity No. & Activity Title & Description \\
\hline 6.7 .2 .5 .2 .2 .01 & $\begin{array}{l}\text { QAS\&A Management \& } \\
\text { Administration }\end{array}$ & $\begin{array}{l}\text { Management and administrative activities that support the QA program and the } \\
\text { operation of the QA Standards \& Administration organization. }\end{array}$ \\
\hline 6.7 .2 .5 .2 .2 .02 & Program Planning & $\begin{array}{l}\text { This activity is comprised of Site Support Program Plan development, Activity } \\
\text { Data Sheet Development, and other program planning related activities. }\end{array}$ \\
\hline 6.7 .2 .5 .2 .2 .03 & $\begin{array}{l}\text { Site Management } \\
\text { System Reporting }\end{array}$ & $\begin{array}{l}\text { This activity includes data compilation and report generation to satisfy the } \\
\text { requirements of the Site Management System Reporting criteria for Quality } \\
\text { Assurance. Al so included in this task are RL two-and-six-month award fee } \\
\text { activities and management review activities. }\end{array}$ \\
\hline 6.7 .2 .5 .2 .2 .04 & $\begin{array}{l}\text { QA Manual } \\
\text { Maintenance }\end{array}$ & $\begin{array}{l}\text { This activity includes maintenance \& administration of WHC QA policies, } \\
\text { procedures and manuals. }\end{array}$ \\
\hline 6.7 .2 .5 .2 .2 .05 & RL Interface & $\begin{array}{l}\text { This activity includes coordinating } \mathrm{RL} / \text { WHC bi-weekly interface meeting, action } \\
\text { item tracking and statusing, and report generation. }\end{array}$ \\
\hline 6.7 .2 .5 .2 .3 .01 & $\begin{array}{l}\text { Projects and } \\
\text { Procurement } \\
\text { Management \& } \\
\text { Administration }\end{array}$ & $\begin{array}{l}\text { Management and administrative activities that support the } Q A \text { program and the } \\
\text { operation of the Projects and Procurement organization. }\end{array}$ \\
\hline $6.7 .2 .5 \cdot 2 \cdot 3.02$ & QA Support of ESQ & $\begin{array}{l}\text { This activity includes field support of ESQ programs including Health Physics } \\
\text { (Radiological Engineering, Operational ALARA Engineering, HP Policy \& } \\
\text { Procedures, HP Dosimetry, Dosimetry Administration), Industrial Health, Safety \& } \\
\text { Fire Protection, ESQ Compliance (ESQ Data, Quality Compliance Assurance, } \\
\text { Environmental Compliance Assurance, Safety Compliance Assurance), Quality } \\
\text { Assurance Oversight, Nuclear Safety, Security (excluding Safeguards activities), } \\
\text { Fire Department \& Fire Systems Maintenance and Emergency Preparedness. }\end{array}$ \\
\hline 6.7 .2 .5 .3 .1 & TWRS QA & $\begin{array}{l}\text { Activities include independent quality control, quality engineering, and } \\
\text { oversight functions necessary to support the TWRS project facilities including } \\
\text { the Tank Farms, } 242-A \text { Evaporator, Grout, HWVP, and other TWRS facilities. }\end{array}$ \\
\hline
\end{tabular}




\begin{tabular}{|c|c|c|}
\hline $\begin{array}{l}\text { Description } \\
\text { of } \\
\text { Activities }\end{array}$ & \multicolumn{2}{|c|}{$\begin{array}{l}\text { Westinghouse Hanford Company } \\
\text { Quality Assurance } \\
6.7 .2 .5\end{array}$} \\
\hline Activity No. & Activity Title & Description \\
\hline 6.7 .2 .5 .3 .2 & $\begin{array}{l}\text { QA Standards and } \\
\text { Administration } \\
\text { Direct Support }\end{array}$ & $\begin{array}{l}\text { Activities includes quality control training support, documents control and } \\
\text { records management, and } Q A \text { training support. }\end{array}$ \\
\hline 6.7 .2 .5 .3 .3 & $\begin{array}{l}\text { Spent Nuclear Fuel } \\
\text { QA Direct Support }\end{array}$ & $\begin{array}{l}\text { Activities include independent quality control, quality engineering, and } \\
\text { oversight functions necessary to support the Spent Nuclear Fuels project } \\
\text { facilities including the K-Basins and other spent fuel activities. }\end{array}$ \\
\hline 6.7 .2 .5 .3 .4 & $\begin{array}{l}\text { Transition } \\
\text { Projects QA Direct } \\
\text { Support }\end{array}$ & $\begin{array}{l}\text { Activities include independent quality control, quality engineering, and } \\
\text { oversight functions necessary to support the Transition Projects program } \\
\text { facilities including PFP, FFTF, PUREX, B-Plant, and Fuel Fabrication Facilities } \\
\text { Transition Projects. }\end{array}$ \\
\hline 6.7 .2 .5 .3 .5 & $\begin{array}{l}\text { Waste, Analytical } \\
\text { and Environmental } \\
\text { QA Direct Support }\end{array}$ & $\begin{array}{l}\text { Activities include independent quality control, quality engineering, and } \\
\text { oversight functions necessary to support the Waste, Analytical and Environmental } \\
\text { program facilities including the } 222-S \text { Analytical Laboratories, the Central } \\
\text { Waste Complex, T-Plant, and TRUSAF. }\end{array}$ \\
\hline 6.7 .2 .5 .3 .6 & $\begin{array}{l}\text { Projects and } \\
\text { Procurement Direct } \\
\text { Support }\end{array}$ & $\begin{array}{l}\text { Activities include independent quality control, quality engineering, and } \\
\text { oversight functions necessary to support the HEPA Filter Test Facility, } \\
\text { Fabrication Shop, supplier evaluations, support for Stores, receiving } \\
\text { inspection, and Construction Projects. }\end{array}$ \\
\hline
\end{tabular}




\begin{tabular}{|c|c|c|c|c|}
\hline \multicolumn{3}{|c|}{ 2.B.1 Milestone List } & \multicolumn{2}{|c|}{$\begin{array}{l}\text { FY } 1995 \\
\text { Site Support Program Plan } \\
\text { Date Prepared: } 09 / 16 / 94\end{array}$} \\
\hline \multicolumn{2}{|c|}{ Milestone } & \multirow{2}{*}{$\begin{array}{c}\text { WBS } \\
\text { Number }\end{array}$} & \multirow{2}{*}{ Milestone Description } & \multirow{2}{*}{$\begin{array}{c}\text { Scheduled } \\
\text { Completion } \\
\text { Date }\end{array}$} \\
\hline Type & Number & & & \\
\hline RL & QAD-95-001 & 6.7.2.5.1.1.02 & WHC Self-Assessment Implementation Plan Update & $01 / 31 / 95$ \\
\hline RL & QAD-95-002 & 6.7.2.5.1.1.02 & Mid-Year WHC Self-Assessment Report & $02 / 15 / 95$ \\
\hline RL & QAD-95-003 & 6.7.2.5.1.1.02 & Annual WHC Self-Assessment Report & $08 / 15 / 95$ \\
\hline RL & QAD-95-004 & 6.7.2.5.2.2.02 & Issue FY 1996 QA SSPP & $08 / 31 / 95$ \\
\hline RL & QAD-95-005 & 6.7.2.5.2.2.04 & $\begin{array}{l}\text { QA Program Description \& Implementation Plan for Rule } \\
\text { 10CFR830.120 }\end{array}$ & $11 / 04 / 94$ \\
\hline CNTR & QAD-95-006 & 6.7.2.5.2.2.04 & $\begin{array}{l}\text { Instructions for Preparation of Qual ity Assurance Program } \\
\text { Plans Based on Rule 10CFR830.120 }\end{array}$ & $11 / 04 / 94$ \\
\hline CNTR & QAD-95-007 & 6.7.2.5.1.2.01 & $\begin{array}{l}\text { First FY } 1995 \text { Semi-Annual Projects and Procurement Oversight } \\
\text { Support Schedule }\end{array}$ & $10 / 05 / 94$ \\
\hline CNTR & QAD-95-008 & 6.7.2.5.1.2.01 & $\begin{array}{l}\text { Second FY } 1995 \text { Semi-Annual Projects and Procurement Oversight } \\
\text { Support Schedule }\end{array}$ & $04 / 05 / 95$ \\
\hline CNTR & OAD-95-009 & 6.7.2.5.1.1.01 & $\begin{array}{l}\text { First FY } 1995 \text { Semi-Annual Quality Assurance Standards and } \\
\text { Administration Oversight Schedule }\end{array}$ & $10 / 05 / 94$ \\
\hline CNTR & QAD-95-010 & 6.7.2.5.1.1.01 & $\begin{array}{l}\text { Second FY } 1995 \text { Semi-Annual ouality Assurance Standards and } \\
\text { Administration Oversight Schedule }\end{array}$ & $04 / 05 / 95$ \\
\hline CNTR & QAD-95-011 & 6.7.2.5.1.2.01 & Evaluated Supplier List Monthly Update & $10 / 14 / 94$ \\
\hline CNTR & QAD-95-012 & 6.7 .2 .5 .1 .2 .01 & Evaluated Supplier List Monthly Update & $11 / 15 / 94$ \\
\hline CNTR & QAD-95-013 & 6.7.2.5.1.2.01 & Evaluated Suppl ier List Monthly Update & $12 / 15 / 94$ \\
\hline CNTR & QAD- $95-014$ & 6.7.2.5.1.2.01 & Evaluated Supplier List Monthly Update & $01 / 16 / 95$ \\
\hline CNTR & QAD- $95-015$ & 6.7.2.5.1.2.01 & Evaluated Supplier List Monthly Update & $02 / 15 / 95$ \\
\hline CNTR & QAD-95-016 & 6.7.2.5.1.2.01 & Evaluated Supplier List Monthly Update & $03 / 15 / 95$ \\
\hline
\end{tabular}




\begin{tabular}{|c|c|c|c|c|}
\hline \multicolumn{3}{|c|}{ 2.B.1 Milestone List } & $\begin{array}{c}\text { Westinghouse Hanford Company } \\
\text { Quality Assurance } \\
6.7 .2 .5\end{array}$ & $\begin{array}{l}\text { FY } 1995 \\
\text { Site Support Program Plan } \\
\text { Date Prepared: } 09 / 16 / 94\end{array}$ \\
\hline \multicolumn{2}{|c|}{ Milestone } & \multirow{2}{*}{$\begin{array}{c}\text { WBS } \\
\text { Number }\end{array}$} & \multirow{2}{*}{ Milestone Description } & \multirow{2}{*}{$\begin{array}{c}\text { Scheduled } \\
\text { Completion } \\
\text { Date }\end{array}$} \\
\hline Type & Number & & & \\
\hline CNTR & QAD-95-017 & 6.7 .2 .5 .1 .2 .01 & Evaluated Supplier List Monthly Update & $04 / 14 / 95$ \\
\hline CNTR & QAD-95-018 & 6.7 .2 .5 .1 .2 .01 & Evaluated Supplier List Monthly Update & $05 / 15 / 95$ \\
\hline CNTR & QAD-95-019 & 6.7 .2 .5 .1 .2 .01 & Evaluated Supplier List Monthly Update & $06 / 15 / 95$ \\
\hline CNTR & QAD- $95-020$ & $6.7 .2 .5 \cdot 1.2 .01$ & Evaluated Supplier List Monthly Update & $07 / 14 / 95$ \\
\hline CNTR & QAD- $95-021$ & 6.7 .2 .5 .1 .2 .01 & Evaluated Supplier List Monthly Update & $08 / 15 / 95$ \\
\hline CNTR & QAD-95-022 & 6.7 .2 .5 .1 .2 .01 & Evaluated Supplier List Monthly Update & $09 / 15 / 95$ \\
\hline RL & QAD- $95-023$ & 6.7 .2 .5 .1 .6 .02 & Annual Suspect/Counterfeit Products Report & $01 / 31 / 95$ \\
\hline & & & - & \\
\hline
\end{tabular}




\begin{tabular}{|c|c|c|}
\hline 2.C.1 & Westinghouse Hanford Company & FY 1995 \\
Cost Baseline by Program Element & Quality Assurance & Site Support Program Plan \\
D.7.2.5 & Date Prepared: 09/16/94 \\
\hline
\end{tabular}

FY 1994 Cost Baseline ( $\$$ s in 000s)

\begin{tabular}{|c|c|c|}
\hline $\begin{array}{c}\text { WBS No. (FY } \\
\text { 1994) }\end{array}$ & Title & Total \$s \\
\hline \multirow[t]{2}{*}{6.7 .2 .5 .2 .2} & QA Standards \& Administration - Oversight Support & \\
\hline & Projects \& Procurement QA - Oversight Support & \\
\hline \multirow[t]{4}{*}{6.7 .2 .1 .2 .1} & QA Program - Overhead Support & \\
\hline & QA Standards \& Administration - Overhead Support & \\
\hline & Projects \& Procurement - Overhead Functions & \\
\hline & FY 1994 Total for $6.7 .2 .1 .2 .1 \& .2$ & 11969 \\
\hline
\end{tabular}




\begin{tabular}{|c|c|c|}
\hline $\begin{array}{c}\text { 2.C.1 } \\
\text { Cost Baseline by Program Element }\end{array}$ & Westinghouse Hanford Company & $\begin{array}{c}\text { Fy } 1995 \\
\text { Site Support Program Plan } \\
\text { Datity Assurance } \\
6.7 .2 .5\end{array}$ \\
\hline
\end{tabular}

FY 1995 Cost Baseline (\$s in 000s)

\begin{tabular}{|c|c|c|c|}
\hline WBS No. & Title & & Total \$s \\
\hline 6.7 .2 .5 .1 & Quality Assurance Overhead Support & & 3,100 \\
\hline 6.7 .2 .5 .2 & Quality Assurance Oversight Support & & 1,940 \\
\hline 6.7 .2 .5 .3 & Quality Assurance Direct Support & & 14,607 \\
\hline & & FY 1995 Total for 6.7 .2 .5 & 19,647 \\
\hline
\end{tabular}




\begin{tabular}{|c|c|c|}
\hline 2.C.1 & Westinghouse Hanford Company & $\begin{array}{c}\text { FY 1995 } \\
\text { Site Support Program Plan } \\
\text { Duality Assurance } \\
6.7 .2 .5\end{array}$ \\
\hline
\end{tabular}

FY 1996 Cost Baseline (\$s in 000s)

\begin{tabular}{|c|c|c|c|}
\hline WBS No. & Title & & Total \$s \\
\hline 6.7 .2 .5 .1 & Quality Assurance Overhead Support & & 3,193 \\
\hline 6.7 .2 .5 .2 & Quality Assurance Oversight Support & & 1,998 \\
\hline 6.7 .2 .5 .3 & Quality Assurance Direct Support & & 15,045 \\
\hline & & FY 1996 Total for 6.7 .2 .5 & 20,236 \\
\hline
\end{tabular}




\begin{tabular}{|c|c|c|}
\hline 2.C.1 & Westinghouse Hanford Company & $\begin{array}{c}\text { FY } 1995 \\
\text { Site Support Program P1an } \\
\text { Quality Assurance } \\
6.7 .2 .5\end{array}$ \\
Date Prepared: $09 / 16 / 94$
\end{tabular}

Fy 1997 Cost Baseline (\$s in 000s)

\begin{tabular}{|c|c|c|c|}
\hline WBS No. & Title & & Total \$s \\
\hline 6.7 .2 .5 .1 & Quality Assurance 0verhead Support & & 3289 \\
\hline 6.7 .2 .5 .2 & Quality Assurance Oversight Support & & 2058 \\
\hline 6.7 .2 .5 .3 & Qual ity Assurance Direct Support & & 15,496 \\
\hline & . & FY 1997 Total for 6.7 .2 .5 & 20,843 \\
\hline
\end{tabular}


2.C. 1.1

Cost Baseline by Funding Source
Westinghouse Hanford Company

Quality Assurance

$$
6.7 .2 .5
$$

FY 1995

Site Support Program Plan Date Prepared: 09/16/94

\begin{tabular}{|c|c|c|c|c|c|c|c|}
\hline WBS \# & Title & CSP /OS & SLP & $G \& A$ & Direct $\$$ & Total \$s & Dept. OH \\
\hline 6.7 .2 .5 .1 & QA Oversight Support & 7051 & & & & 7051 & \\
\hline 6.7 .2 .5 .2 & QA Overhead Support & & & & & .0 & 4918 \\
\hline \multirow[t]{5}{*}{6.7 .2 .5 .3} & QA Direct Support & & & & 10000 & $10,000.0$ & \\
\hline & & & & & & 0.0 & \\
\hline & & & & & & 0.0 & \\
\hline & & & & & & 0.0 & \\
\hline & & & & & & 0.0 & \\
\hline & Totals & 7051 & 0.0 & 0.0 & $10,000.0$ & 17051.0 & $4,918.0$ \\
\hline
\end{tabular}

Fy 1995 Cost Baseline ((Dollars in 000s)

\begin{tabular}{|c|c|c|c|c|c|c|c|}
\hline WBS \# & Title & $\operatorname{csp} / 0 S$ & SLP & $G \& A$ & Direct $\$$ & Total \$s & Dept. $\mathrm{OH}$ \\
\hline 6.7 .2 .5 .1 & QA Oversight Support & 1940 & & & & 1940 & \\
\hline 6.7 .2 .5 .2 & OA Overhead Support & & & & & 0.0 & 3100 \\
\hline \multirow[t]{5}{*}{6.7 .2 .5 .3} & QA Direct Support & & & & & 0.0 & \\
\hline & & & & & & 0.0 & \\
\hline & & & & & & 0.0 & \\
\hline & & & & & & 0.0 & \\
\hline & & & & & & 0.0 & \\
\hline & Totals & 1940.0 & 0.0 & 0.0 & 0.0 & 1940 & 3100.0 \\
\hline
\end{tabular}




\begin{tabular}{|c|c|c|}
\hline $\begin{array}{c}\text { 2.C.1.1 } \\
\text { Cost Baseline by Funding Source }\end{array}$ & $\begin{array}{c}\text { Westinghouse Hanford Company } \\
\text { Qual ity Assurance } \\
6.7 .2 .5\end{array}$ & $\begin{array}{l}\text { FY } 1995 \\
\text { Site Support Program Plan } \\
\text { Date Prepared: } 09 / 16 / 94\end{array}$ \\
\hline
\end{tabular}

\begin{tabular}{|c|c|c|c|c|c|c|c|}
\hline WBS \# & Title & $\operatorname{csp} / 0 \mathrm{~S}$ & SLP & $G \& A$ & Direct $\$$ & Total \$s & Dept, OH \\
\hline 6.7 .2 .5 .1 & Oversight Support & 2029 & & & & 2029 & \\
\hline 6.7 .2 .5 .2 & Overhead Support & & & & & 0.0 & 3233 \\
\hline \multirow[t]{5}{*}{6.7 .2 .5 .3} & Direct Support & & & & & 0.0 & \\
\hline & & & & & & 0.0 & 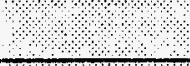 \\
\hline & & & & & & 0.0 & \\
\hline & & & & & & 0.0 & 3 \\
\hline & & & & & & 0.0 & 1,8 \\
\hline & Totals & 2029 & 0.0 & 0.0 & 0.0 & 2029.0 & 3233.0 \\
\hline
\end{tabular}

\begin{tabular}{|c|c|c|c|c|c|c|c|c|}
\hline WBS \# & Title & & $\mathrm{CSP} / 0 \mathrm{~S}$ & SLP & $G \& A$ & Direct $\$$ & Total \$s & Dept. OH \\
\hline 6.7 .2 .5 .1 & Oversight Support & & 2090 & & & & 2090 & \\
\hline 6.7 .2 .5 .2 & Overhead Support & & & & & & 0.0 & 3329 \\
\hline \multirow[t]{5}{*}{6.7 .2 .5 .3} & Direct Support & & & & & & 0.0 & 4 \\
\hline & & & & & & & 0.0 & . \\
\hline & . & & & & & & 0.0 & 4 \\
\hline & & & & & & & 0.0 & 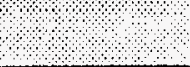 \\
\hline & & & & & & & 0.0 & 8 \\
\hline & . & Totals & 2090 & 0.0 & 0.0 & 0.0 & 2090 & 3329 \\
\hline
\end{tabular}




\begin{tabular}{|c|c|c|}
\hline 2.C.2 Cost Basis & $\begin{array}{c}\text { Westinghouse Hanford Company } \\
\text { Qual ity Assurance } \\
6.7 .2 .5\end{array}$ & $\begin{array}{c}\text { Fy 1995 } \\
\text { Site Support Program Plan } \\
\text { Date Prepared: 09/16/94 }\end{array}$ \\
\hline
\end{tabular}

Section 1.B.1, Internal Assessment Summary, Section 2.A.2, Description of Activities, and Section 3.A, WBS Dictionary Sheets, of this SSPP provide detailed descriptions of the products/services provided by the Quality Assurance organization.

The overall cost of the Quality Assurance program for FY 1995 is estimated to be $\$ 19,640,000$, while the Quality Assurance program elements will require funding at the following levels:

- $\quad$ Oversight Support....\$1,940,000

- $\quad$ Overhead Support....\$3,100,000

- $\quad$ Direct Support......\$14,600,000

The cost estimates reflect a change in the method of cost allocation from FY 1994 . The majority of costs associated with supporting the Direct Programs will be funded by Direct Programs in FY 1995, instead of overhead/Oversight pools. This has resulted in a reduction of the overhead rate to approximately $25 \%$ from $44 \%$.

The varied types and scope of products, services, and customers make it impractical to define unit costs at this time. However, the Quality Assurance organization will attempt to standardize cost accounting practices during FY 1995 in order to better define unit costs for FY 1996 planning purposes. History and analogy were used to develop the cost estimate.

Significant changes are anticipated in the volume and costs associated with Quality Assurance products and services. This is due to the implementation of an Environmental Restoration Contract by the DOE (15 ful1-time equivalents) and productivity improvements ( 7 ful1-time equivalents). Other scope increases (5 ful1-time equivalents) will partially off set the previous reductions in work scope for a net reduction in full-time equivalents of 17 in FY 1995. 


\begin{tabular}{|c|c|c|c|c|c|c|c|c|}
\hline 2.C.3 FTE Furecasts & \multicolumn{5}{|c|}{$\begin{array}{c}\text { Westinghouse Hanford Company } \\
\text { Qual ity Assurance } \\
6.7 .2 .5\end{array}$} & \multicolumn{3}{|c|}{$\begin{array}{c}\text { FY } 1995 \\
\text { Site Support Program Plan } \\
\text { Date Prepared: } 09 / 16 / 94\end{array}$} \\
\hline \multicolumn{9}{|c|}{ JOB FAMILY - Full Time Equivalent Staff by Job Description } \\
\hline Job category & 1994 & 1995 & 1996 & 1997 & 1998 & 1999 & 2000 & 2001 \\
\hline \multicolumn{9}{|l|}{ MANAGERS } \\
\hline First line & 19 & 16 & 16 & & & & & \\
\hline General/executive & 1 & 1 & 1 & & & & & \\
\hline Project/Program & 5 & 6 & 6 & & & & 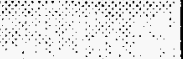 & 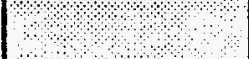 \\
\hline \multicolumn{9}{|l|}{ other } \\
\hline Subtotal Managers & 25.0 & 23.0 & 23.0 & 23.0 & 23.0 & 23.0 & 23.0 & 23.0 \\
\hline \multicolumn{9}{|l|}{ ENGINEERS } \\
\hline \multicolumn{9}{|l|}{ Chemical } \\
\hline \multicolumn{9}{|l|}{ civil } \\
\hline \multicolumn{9}{|l|}{ Computer Sof tware } \\
\hline \multicolumn{9}{|l|}{ Electrical } \\
\hline \multicolumn{9}{|l|}{ Envi ronmental } \\
\hline \multicolumn{9}{|l|}{ Industrial } \\
\hline \multicolumn{9}{|l|}{ Mechanical } \\
\hline \multicolumn{9}{|l|}{ Nucl ear } \\
\hline Plant & 22 & 22 & 22 & & & & & 18. \\
\hline Quality Control & 120.5 & 108 & 108 & & & & & 3134 \\
\hline \multicolumn{9}{|l|}{ Safety } \\
\hline \multicolumn{9}{|l|}{ other } \\
\hline Subtotal Engineers & 142.5 & 130 & 130 & 130 & 130 & 130 & 130 & 130 \\
\hline
\end{tabular}




\begin{tabular}{|c|c|c|c|c|c|c|c|c|}
\hline 2.C.3 FTE Forecasts & \multicolumn{4}{|c|}{$\begin{array}{c}\text { West inghouse Hanford Company } \\
\text { Qual ity As surance } \\
6.7 .2 .5\end{array}$} & & \multicolumn{3}{|c|}{$\begin{array}{c}\text { FY } 1995 \\
\text { Site Support Program Plan } \\
\text { Date Prepared: } 09 / 16 / 94\end{array}$} \\
\hline \multicolumn{9}{|c|}{ JOB FAMILY - Full Time Equivalent Staff by Job Description } \\
\hline Job category & 1994 & 1995 & 1996 & 1997 & 1998 & 1999 & 2000 & 2001 \\
\hline \multicolumn{9}{|l|}{ scientists } \\
\hline \multicolumn{9}{|l|}{ Chemists } \\
\hline Envi ronmental & 0 & 1 & 1 & & & & & \\
\hline \multicolumn{9}{|l|}{ Life } \\
\hline \multicolumn{9}{|l|}{ Mathematicians } \\
\hline \multicolumn{9}{|l|}{ Physicists Health } \\
\hline \multicolumn{9}{|l|}{ Social Industrial Hygienist } \\
\hline \multicolumn{9}{|l|}{ other } \\
\hline Subtotal scientists & 0.0 & 1.0 & 1.0 & 1.0 & 1.0 & 1.0 & 1.0 & 1.0 \\
\hline \multicolumn{9}{|l|}{ ADMIN/OTHER PROFESSIONALS } \\
\hline \multicolumn{9}{|l|}{ Accountant/auditor } \\
\hline \multicolumn{9}{|l|}{ Communications Special ist } \\
\hline Compliance inspectors & 2 & 2 & 2 & & & & & \\
\hline \multicolumn{9}{|l|}{ Computer System Anal } \\
\hline \multicolumn{9}{|l|}{ Cost Est/planner/sch } \\
\hline \multicolumn{9}{|l|}{ Heal th Physics Analyst } \\
\hline \multicolumn{9}{|l|}{ Industrial Hygiene } \\
\hline \multicolumn{9}{|l|}{ Safeguard \& Security } \\
\hline \multicolumn{9}{|l|}{ Trainers } \\
\hline other & 1 & 1 & 1 & & & & & \\
\hline
\end{tabular}




\begin{tabular}{|c|c|c|c|c|c|c|c|c|}
\hline 2.C. 3 FTE Forecasts & \multicolumn{5}{|c|}{$\begin{array}{c}\text { West inghouse Hanford Company } \\
\text { Quality Assurance } \\
6.7 .2 .5\end{array}$} & \multicolumn{3}{|c|}{$\begin{array}{c}\text { FY } 1995 \\
\text { Site Support Program Plan } \\
\text { Date Prepared: } 09 / 16 / 94\end{array}$} \\
\hline \multicolumn{6}{|c|}{ JOB FAMILY - Full Tịme Equivalent Staff by Job Description } & \multicolumn{3}{|c|}{ NOTE: Job Family Only After 1996} \\
\hline Job category & 1994 & 1995 & 1996 & 1997 & 1998 & 1999 & 2000 & 2001 \\
\hline $\begin{array}{r}\text { Subtotal Admin/other } \\
\text { Professionals }\end{array}$ & 3.0 & 3.0 & 3.0 & 3.0 & 3.0 & 3.0 & 3.0 & 3.0 \\
\hline \multicolumn{9}{|l|}{ GEN ADM/SECRETARY/CLERK } \\
\hline Admin Assistants & 0 & 1 & 1 & & & 3 & & ? \\
\hline office Clerks (Gen) & 3 & 2 & 2 & & \% & & & \\
\hline office Clerks (Special) & 2 & 1 & 1 & & & & & \\
\hline Secretaries & 18 & 16 & 16 & & & & & \\
\hline \multicolumn{9}{|l|}{ Typist/Word Process } \\
\hline \multicolumn{9}{|l|}{ other } \\
\hline Subtotal Gen Adm/Secretary/Clerk & 23.0 & 20.0 & 20.0 & 20.0 & 20.0 & 20.0 & 20.0 & 20.0 \\
\hline \multicolumn{9}{|l|}{ TECHNICIANS } \\
\hline \multicolumn{9}{|l|}{ Computer Oper/Coder } \\
\hline Engrs/Tech & 32 & 27 & 27 & & & & & \\
\hline \multicolumn{9}{|l|}{ Envir. Sci Technicians } \\
\hline \multicolumn{9}{|l|}{ Heal th Phys. Technic. } \\
\hline \multicolumn{9}{|l|}{ Indus. Saf/Heal th Tech } \\
\hline \multicolumn{9}{|l|}{ Instru/Control Tech } \\
\hline \multicolumn{9}{|l|}{ other } \\
\hline Subtotal Technicians & 32.0 & 27.0 & 27.0 & 27.0 & 27.0 & 27.0 & 27.0 & 27.0 \\
\hline
\end{tabular}




\begin{tabular}{|c|c|c|c|c|c|c|c|c|}
\hline 2.C.3 FTE Forecasts & \multicolumn{4}{|c|}{$\begin{array}{c}\text { Westinghouse Hanford Company } \\
\text { Quality Assurance } \\
6.7 .2 .5\end{array}$} & & \multicolumn{3}{|c|}{$\begin{array}{l}\text { FY } 1995 \\
\text { Site Support Program Plan } \\
\text { Date Prepared: } 09 / 16 / 94\end{array}$} \\
\hline \multicolumn{9}{|c|}{ JOB FAMILY - Full Time Equivalent Staff by Job Description } \\
\hline Job category & 1994 & 1995 & 1996 & 1997 & 1998 & 1999 & 2000 & 2001 \\
\hline \multicolumn{9}{|l|}{ CRAFIS } \\
\hline \multicolumn{9}{|l|}{ Electricians } \\
\hline \multicolumn{9}{|l|}{ other } \\
\hline \multicolumn{9}{|l|}{ Instrument Specialist } \\
\hline \multicolumn{9}{|l|}{ Lacksmi th Safemaster } \\
\hline Subtotal Crafts & 0.0 & 0.0 & 0.0 & 0.0 & 0.0 & 0.0 & 0.0 & 0.0 \\
\hline \multicolumn{9}{|l|}{ LABOR \& GEN WORKERS } \\
\hline \multicolumn{9}{|l|}{ Firefighters } \\
\hline \multicolumn{9}{|l|}{ Security Guards } \\
\hline \multicolumn{9}{|l|}{ other } \\
\hline \multicolumn{9}{|l|}{ Paramedics } \\
\hline Subtotal Labor \& Gen Workers & 0.0 & 0.0 & 0.0 & & & & & \\
\hline Total FrEs 6.7.2.5 & 225.5 & 204 & 204 & 204 & 204 & 204 & 204 & 204 \\
\hline
\end{tabular}




\begin{tabular}{|c|c|c|c|c|c|}
\hline \multirow{6}{*}{$\begin{array}{l}\text { 3.A } \\
\text { Work } \\
\text { Breakdown } \\
\text { Structure } \\
\text { Dictionary }\end{array}$} & \multirow{6}{*}{\multicolumn{3}{|c|}{$\begin{array}{l}\text { Westinghouse Hanford Company } \\
\text { Quality Assurance } \\
\text { Part } 1 \text { - Summary } \\
\text { (Dollars in 000's) }\end{array}$}} & \multirow{6}{*}{$\begin{array}{l}\text { FY } 1995 \text { SSPP } \\
\text { Rev. \# } 0\end{array}$} & Source: \\
\hline & & & & & SWS \\
\hline & & & & & OST \\
\hline & & & & & Mgmt Pro \\
\hline & & & & & DOH \\
\hline & & & & & DIRECT \\
\hline $\begin{array}{r}\text { Cost Account } \\
1 M D M 05\end{array}$ & \multicolumn{3}{|c|}{$\begin{array}{l}\text { Cost Account Title } \\
\text { Quality Assurance Oversight Support }\end{array}$} & \multirow{2}{*}{\multicolumn{2}{|c|}{$\begin{array}{c}\text { Annualized Rate } \\
\text { (For Organizational Overhead and Rated Service } \\
\text { Pool Use Only) }\end{array}$}} \\
\hline SMS WBS 6.7 .2 .5 .1 & \multicolumn{3}{|c|}{ SMS Title: $\quad$ Quality Assurance Oversight Support } & & \\
\hline \multicolumn{4}{|l|}{ CAM Review Approval } & & \multirow{2}{*}{ FY 1995 Rate Request } \\
\hline \multicolumn{4}{|l|}{ SMS Program Manager/Approval } & FY 1994 Rate & \\
\hline \multicolumn{2}{|l|}{ Financial Manager Review/Approval } & \multicolumn{2}{|r|}{ Date } & 0.44 & \multirow{2}{*}{$\begin{array}{c}\text { FY } 1995 \text { Rate Request } \\
0.266 \\
\text { FY } 1995 \text { Approved Rate }\end{array}$} \\
\hline \multicolumn{2}{|l|}{ Responsible Analyst $\quad$ c. J. Mills } & \multirow{2}{*}{\multicolumn{2}{|c|}{$\begin{array}{l}\text { Date } \\
\text { Date }\end{array}$}} & FY 1995 Target Rate & \\
\hline \multirow[t]{2}{*}{ OSBRB Review/Approval } & & & & .266 & \\
\hline & \multicolumn{2}{|c|}{ FY 1994} & \multicolumn{3}{|c|}{ FY 1995} \\
\hline $\begin{array}{l}\text { FULL TIME EQUIVALENTS } \\
\text { (FTES) }\end{array}$ & Budget & $\begin{array}{l}\text { Spending } \\
\text { Forecast (SF) }\end{array}$ & Target & Request & $\begin{array}{l}\text { Approved } \\
\text { Baseline }\end{array}$ \\
\hline . Organizational - Exempt & 55 & 55 & 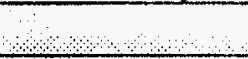 & 18.25 & $2 \ldots$ \\
\hline . Organizational - Nonexempt & 13.5 & 13.5 & & 1.25 & \\
\hline \multicolumn{6}{|l|}{ Organization - Bargaining } \\
\hline Total Organizational FTEs & 68.5 & 68.5 & 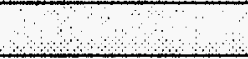 & 19.5 & 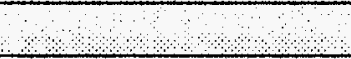 \\
\hline \multicolumn{6}{|l|}{ SUpport FTES } \\
\hline TOTAL FTES & 68.5 & 68.5 & 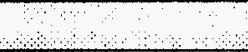 & 19.5 & \\
\hline \multicolumn{6}{|l|}{ COST ELEMENTS } \\
\hline - Labor - Regular & 4657 & 4348 & & 1250.3 & \\
\hline \multicolumn{6}{|l|}{ - Labor Overtime } \\
\hline 0 Total Labor & 4657 & 4348 & $\cdots$ & 1250.3 & \\
\hline 1 Materials & 215 & 60 & & 77 & \\
\hline 2 Purchased Services & 512 & 350 & & 234.1 & \\
\hline 3 Other Hanford & 220 & 100 & & 10 & 5 \\
\hline 4 site Services & 54 & 253 & 4 & 19.2 & 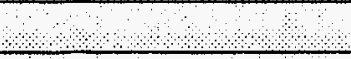 \\
\hline 5 Internal charges & 0 & 0 & & 0 & \\
\hline 6 IRM Support & 54 & 60 & & 12 & \\
\hline 7 Overheads & 2049 & 1880 & & 337.4 & \\
\hline 8 Revenue & & & & & \\
\hline TOTAL DOLLARS & 7761 & 7051 & & 1940 & \\
\hline
\end{tabular}


Breakdown Structure Dictionary

Cost Account No.

1 MDM05

SMS WBS Code:

6.7 .2 .5 .1
Westinghouse W. iord Company

Quality Assurance

Part II - Element Definition
FY 1995

Site Support Program Plan

Date Prepared: 08/24/94

\section{Revision \# 0}

Technical Description/Objectives:

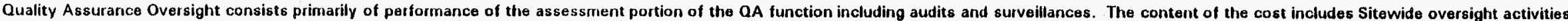
performed by both the QA Standards and Administr ation and the Projects and Procurement QA groups.

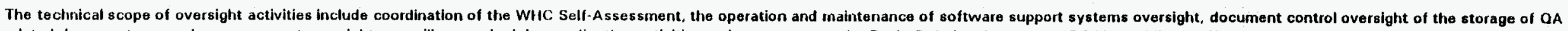

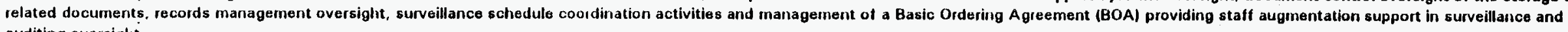
auditing oversighti.

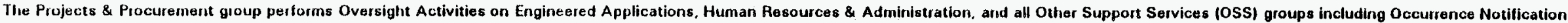
Central Support Services, Site Services and site support programs. Also included is the establishment, maintenance \& auditing functions necessary to support the Approved Suppliers Listing.

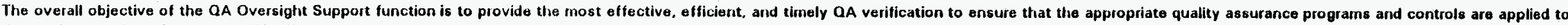
the products and services provided by WHC.

Milestones: (See Section 3.B Milestone Description Sheets for details)

HL WHC Self-Assessment Program Implementation Plan Update, QAD-95-001

HL Mid Year WHC Self. Assessmert Report, QAD.96-002

RL Arrnual WHC Self Assessment Report, QAD-95-003

RL Annual Suspect/Counterfeit Products Report, OAD-95-023

WHC First FY95 Semi-Arnual Projects and Procurement Oversight Support Schedule, QAD-95.007

WHC Second FY95 Semi-Annual Projects and Procurement Oversight Support Schedule, QAD-95-008

WHC First FY95 Semi-Armual QAS\&A Oversight Support Schedule, QAD-95.009

WHC Second FY95 Semi-Annual CAS\&A Oversight Support Schedule, QAD-95-010

WHC Evaluated Supplier List Monthly update, QAD-95-011 thu QAD-95-022 
Work Scope Detailed Description:

QA Standards and Administration Oversight Support (WBS 6.7.2.5.1.1)

Activities associated with the following:

6.7.2.5.1.1.01. QA Standards and Administration - Includes day to day management of oversight activities and the administrative functions required to perform the activities. Also included in this element are the non-labor cost elements.

6.7 25.1.1.02. WHC Self-Assessment - Includes coordinating all related activities within WHC, maintenance of the Self. Assessment Plans. preparation of the WHC Semi-Annual and Annual Self-Assessment Reports and preparing and maintaining WHC policles \& procedures governing the Self-Assessment program.

6.7.2.5.1.1.03. WHC Soltware Oversight - Includes providing oversight of and support to WHC software development, maintenance and operation. Includes planning. scheduling, performing and updating the surveillances, follow up of findings and assurance of problem resolution.

6.7.2.5.1.1.04. WHC Document Control and Records Management - Provides oversight of document control and records management activities by performance of surveillance activities, ldention 6.7.2.5.1.1.05, OA Self Assessment Coordination - The OA self-assessment is planned, structured, documented, and identifies performance strengths and weaknesses and for weaknesses, the root cause and corrective action for the $Q A$ Program. This activity includes providing input to the WHC mid-and-annual status report on self-assessment activities.

Exempt $\cdot 3.75$

Non-Exempt - 0.25 from 6.7.2.5.1.1.0

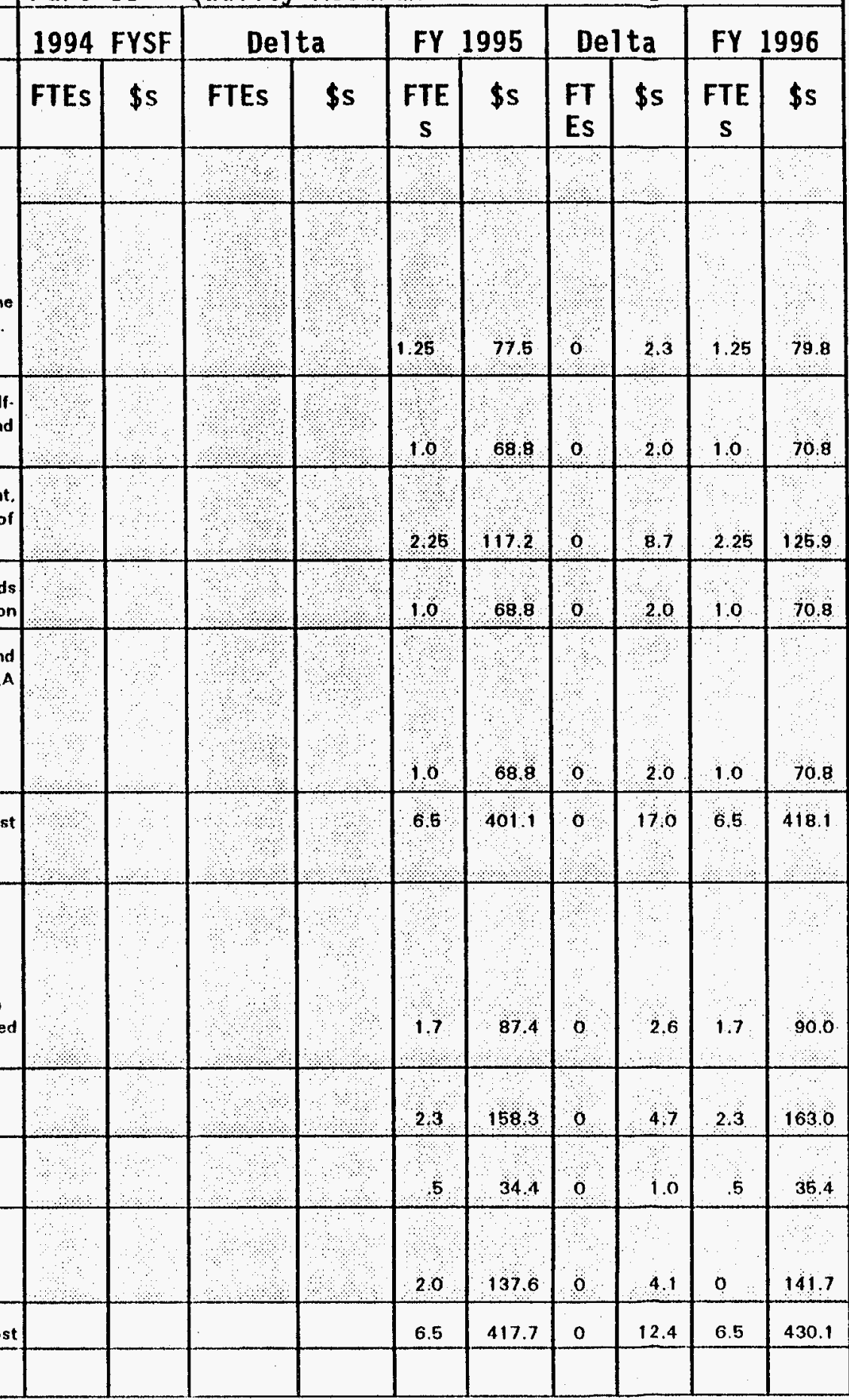

Work Scope Detailed Description:

Projects and Procurement Oversight Support (WBS-6.7.2.5.1.2) Activities associated with the following

6.7. 2.5.1.2.01, Projects \& Procurement QA Management \& Administration - Activities include management, supervision and administration of oversight activities for Projects and Procurement QA Oversight Support and oversight of non-aligned activities.

6.7.2.5.1.2.02, Oversight of ESQ - Activitles include oversight of WHC ESO. Oversight activities include planning, scheduling, and performing surveillances, and the follow up of findings and assurance of problem resolution.

6.7.2.5.1.2.03. Oversight of KEH - Activities include oversight of KEH activities. Oversight activities include planning scheduling, and performing surveillances, and the follow up of findings and assurance of problem resolution.

6.7.2.5.1.2.04. Supplier Audits - Activities include oversight of supplier. Oversight activities include planning scheduling, and performing audits, and the follow up of findings and assurance of problem resolution. 
Element Iask Description

Requests Within Target:

Work Scope Detailed Description

IWHS QA Oversight Support (WBS 6.7.2.5.1.3)

6.7.2.5.1.3.01. Tank Waste Remediation System Audit, Task Team, Assessments "Activities include participation in oversight activities including audits, task team, and, assessments for TWRS.

Spent Nuclear Fuel QA Oversight Support (WBS 6.7.2.5.1.4)

6.7.2.5.1.4.01, Spent Nuclear Fuel Audit, Task Team, Assessment- Activities include participation in oversight activities including audits, task team, and, assessments for the Spent Nuclear Fuel project.

Iransition Projects QA Oversighi Suppoit IWBS 6.7.2.6.1.5)

6.7. 2.5.1.5.01. Transition Projects Audit, Task Team, Assessment - Activities include participation in oversight activities including audits, task team, and, assessments for Transition Projects.

Waste, Analytical and Environmental QA Oversight Suppoit

(WBS 6.7.2.5.1.6)

6.7.2.5.1.6.01, WAE Audit, Task Team, Assossment - Activities include participation in oversight activities including audits, task tearn, and, assessments for Waste. Analytical and Environnental Services.

6.7.2.5.1.6.02. Suspect/counterfeit Parts - This activity the preparation of qualtelly reports that includes a narrative summary of activities which have occurred since the last annual report and a tabulation of the status of each facility-

specific action plan to date.

WHC Labor Cost

o

Contract Labor Cost

Total Labor

\begin{tabular}{|c|c|c|c|c|c|c|c|c|c|}
\hline \multicolumn{2}{|c|}{1994 FYSF } & \multicolumn{2}{|c|}{ Delta } & FY 1995 & Delta & FY & 1996 \\
\hline FTES & \$S & FTES & \$S & $\begin{array}{c}\text { FTE } \\
\text { S }\end{array}$ & \$S & $\begin{array}{c}\text { FT } \\
\text { Es }\end{array}$ & $\begin{array}{c}\text { \$S } \\
\text { FTE } \\
\text { S }\end{array}$ & \$S \\
\hline
\end{tabular}

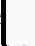

\begin{tabular}{|l|l|l|l|l|l|l|l|l|l|}
\hline 0 & & & 0 & 6.6 & 449.4 & 6.5 & 13.3 & 6.6 & 462.7 \\
\hline
\end{tabular}


Part II - Quality Assurance - 6.7.2.5.1

Element Task Description \begin{tabular}{|l|l|l|l|l|}
1994 FYSF & Delta & FY 1995 & Delta & FY 1996 \\
\hline
\end{tabular}

Requests Within Target:

(List ONLY items that exceed \$5K. (i.e., 20 Computers))

Non-Labor Cost Descriptions

$1 \quad$ Materials FY 1995:

2 Purchased Services FY 1995:

3 Other Hanford FY 1995:

4 Site Services FY 1995:

5 Internal Charges FY 1995:

6 IRM Support FY 1995:

7 Overhead 


\section{A}

Work

Breakdown

Structure

Dictionary
Westinghouse Hanford Company Quality Assurance

Part 1 - Summary

(Dollars in 000 's)
FY 1995 SSPP

Rev. \# 0

\section{Cost Account $1 \mathrm{~J} 3800$ \\ SMS WBS 6.7.2.5.2}

CAM Review Approval

SMS Program Manager/Approval

Financial Manager Review/Approval

Responsible Analyst

c. A. Mills

OSBRB Review/Approval

\section{Cost Account Title}

Quality Assurance Overhead Support

SMS Title: Quality Assurance Overhead Support
Annual ized Rate

(For Organizational Overhead and Rated Service Pool Use On(y)

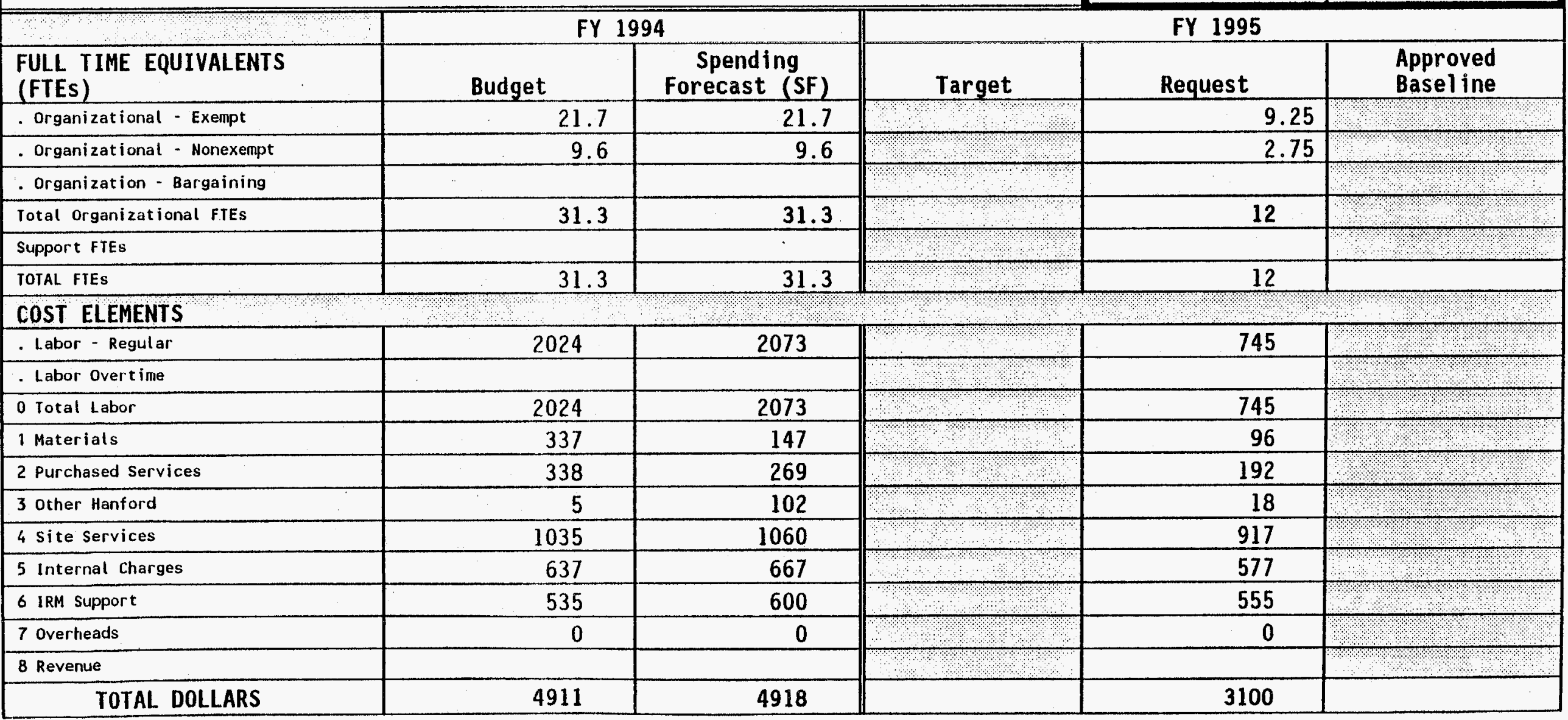




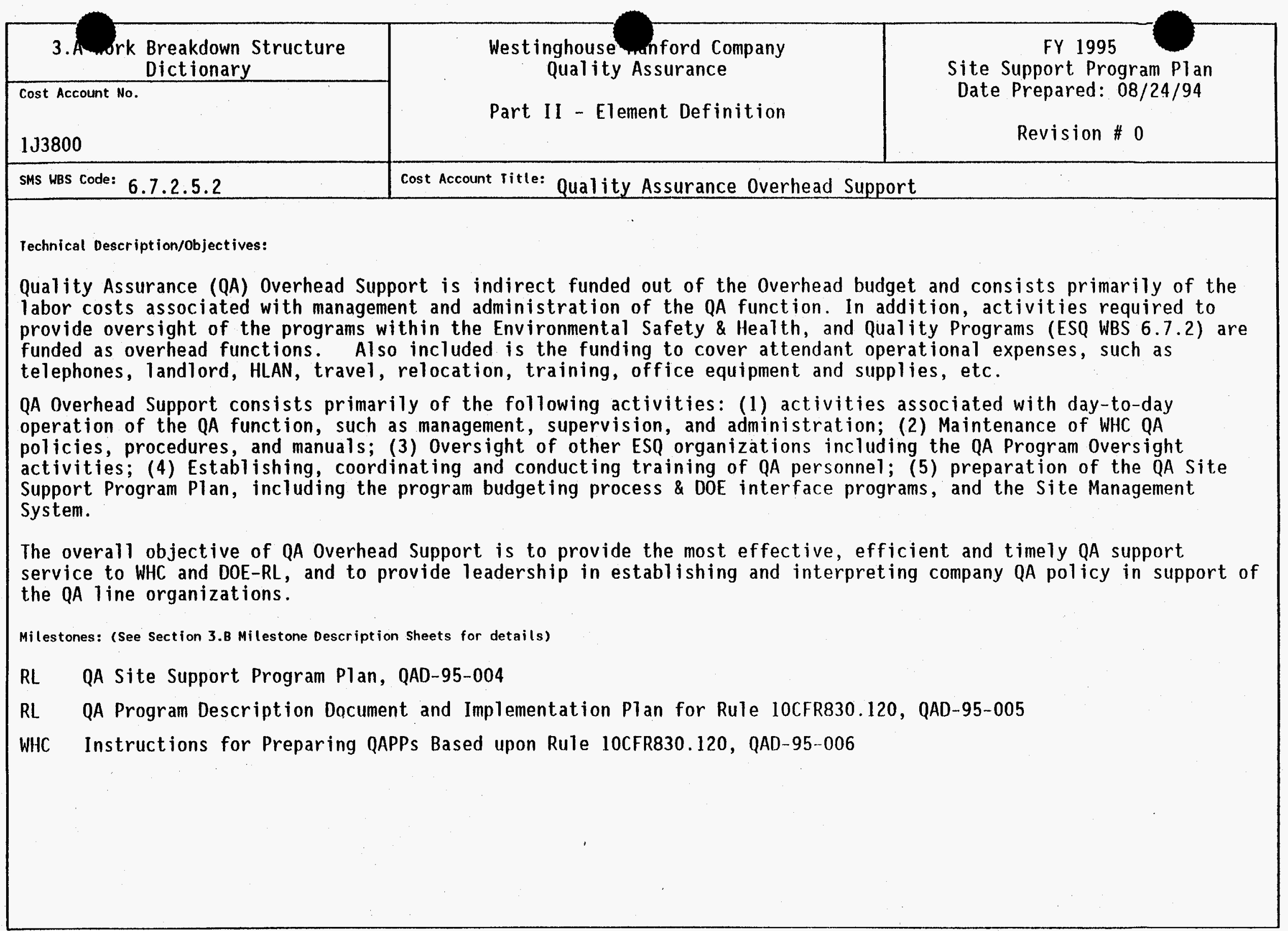


ity Assurance Overliead -6.7 .2 .5 .2$

\section{Element Task Description}

\section{Requests Within Target:}

Work Scope Detailed Description:

QA Program Overhead (WBS $6 \cdot 7 \cdot 2.5 .2 .1)$

QA Program Management \& Administration (WBS-6.7.25.2.1.01) - Management and administrative activities which support the operation of the entire $Q A$ function and its organizations. Included in this activity are costs associated with facilities, equipnent and materials.

WHC Labor Cost Contract Labor Cost

\begin{tabular}{|c|c|c|c|c|c|c|c|c|c|}
\hline 1994 & FYSF & \multicolumn{2}{|c|}{ Delta } & \multicolumn{2}{|c|}{ FY 1995} & \multicolumn{2}{|c|}{ Delta } & \multicolumn{2}{|c|}{ FY 1996} \\
\hline FTES & $\$ s$ & FTES & $\$ s$ & FTES & $\$ s$ & FTES & $\$ s$ & FTEs & $\$ s$ \\
\hline & & & & 2.0 & & 0 & & 20 & $\begin{array}{l}\text { } \\
\text { 111 } 3\end{array}$ \\
\hline & & & & 2.0 & 108.1 & 0 & 3.2 & 2.0 & 111.3 \\
\hline 0 & 0 & 0 & 0 & & & & & & \\
\hline
\end{tabular}

Work Scope Detailed Description:

QA Standards \& Administration Overhead Support (WBS 6.7.2.5.2.2)

QASA Management \& Administration (WBS 6.7.2.5.2.2.011.

Management and administrative activities that support the $Q A$ program and the operation of the $\mathrm{QA}$ Standards \& Administration organization.

Program Planning (WBS 6.7.2.5.2.2.02)

This activity is comprised of Site Support Progran Plan development, Activity Dat a Sheet Development, and other proglam planing related activities.

Site Management System (WBS 6.7 .2 .5 .2 .2 .03 )

This activity includes data compilation and report generation to satisfy the requirements of the Site Managernent System Reporting criteria for Quality Assurance. Also included in this task are Rit two-and-six-month award fee activities and management review activities.

QA Manual Maintenance (WBS 6.7. 2.5. 2.2.04)

This activity includes maintenance $\&$ administration of WHC QA policies, procedures and manuals.

RL Interface (WBS 6.7.2.5.2.2.05)

This activity includes coordinating RL/WHC bi-weekly interface meeting, action item tracking and statusing. and repoit generation.

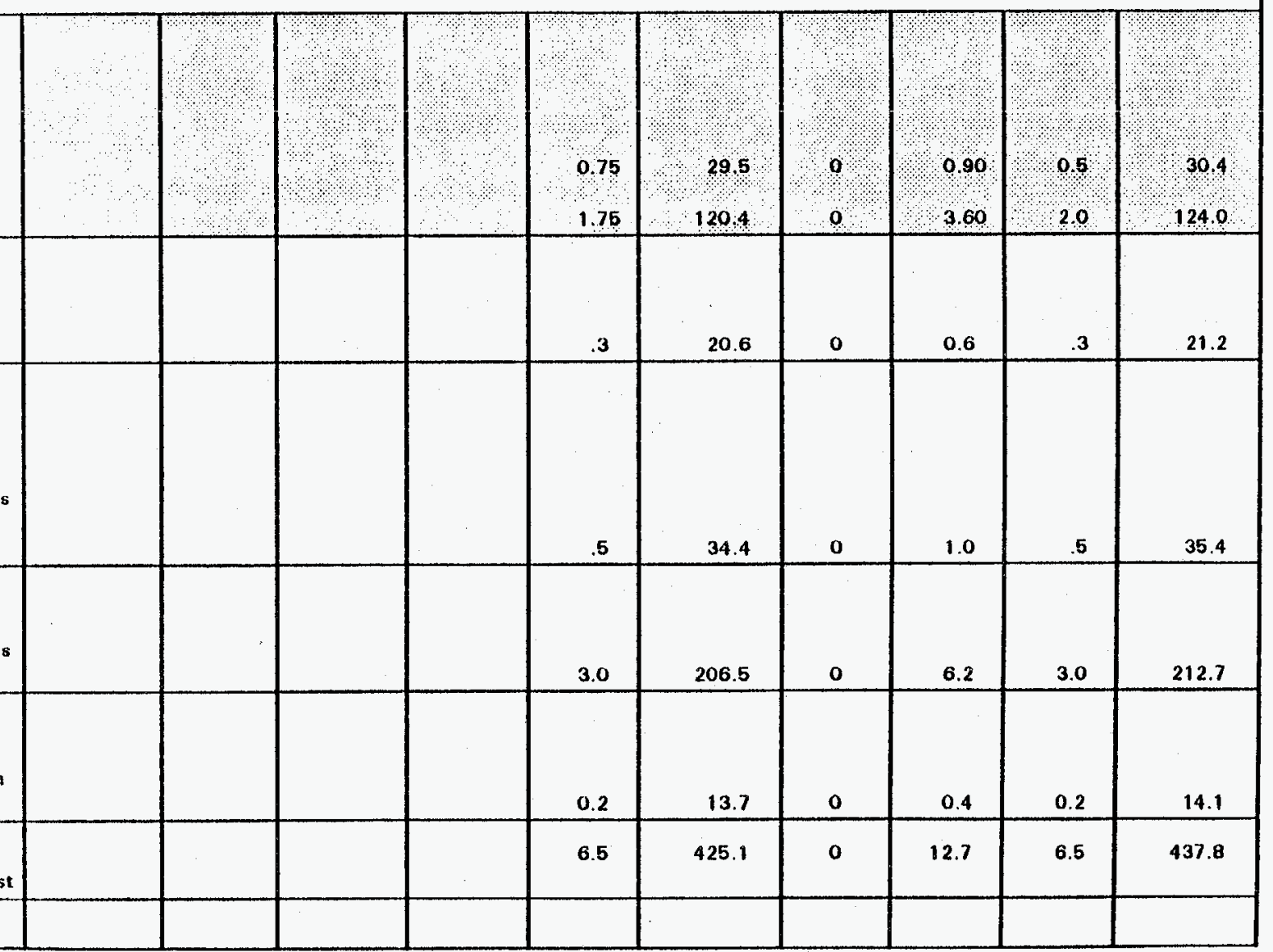




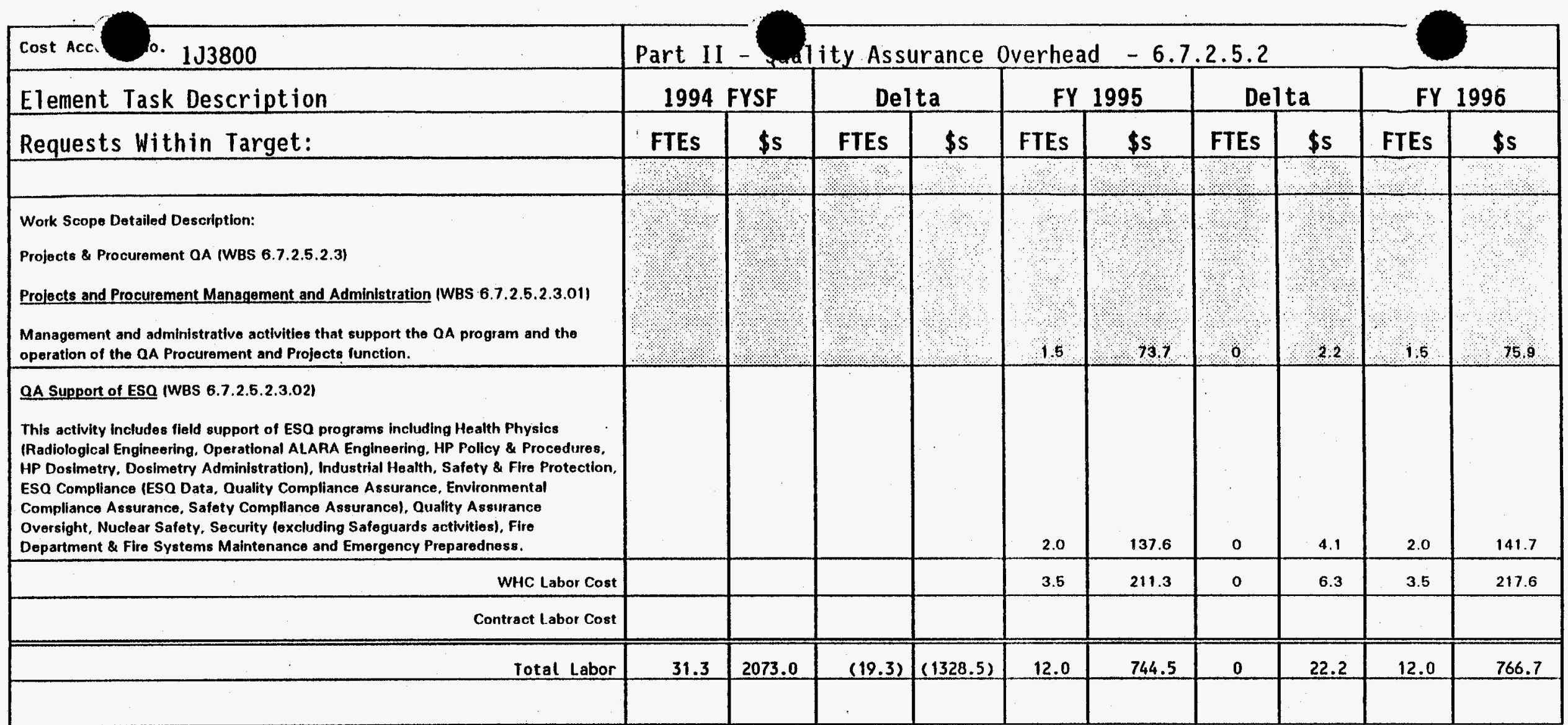




\begin{tabular}{|c|c|c|c|c|c|c|c|c|c|c|}
\hline Element lask Description & \multicolumn{2}{|c|}{1994 FYSF } & \multicolumn{2}{|c|}{ Delta } & \multicolumn{2}{|c|}{ FY 1995} & \multicolumn{2}{|c|}{ Delta } & \multicolumn{2}{|c|}{ FY 1996} \\
\hline Requests Within Target: & FTEs & $\$ s$ & FTEs & $\$ s$ & FTES & $\$ s$ & FTES & $\$ s$ & FTES & $\$ s$ \\
\hline $\begin{array}{l}\text { (list ONIY items that exceed \$5K. (i.e., } 20 \text { Computersl) } \\
\text { Non-Labor Cost Descriptions }\end{array}$ & & & & & & & & & & \\
\hline $\begin{array}{l}1 \text { Materials FY 1995: The purchase of miscellaneous Computer hardware } \\
\text { and software equipment }\end{array}$ & & & & & & 96.0 & & 2.8 & & 98.8 \\
\hline Purchased Services FY 1995: & & & 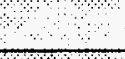 & & & 191.9 & 3 & 5.7 & \% & 197.6 \\
\hline Other Hantord FY 1995: & & & & & & 17.6 & 8 & 0.6 & & 18.1 \\
\hline Site Stuvices FY 1995: & & & & & & 916.7 & & 27.6 & & 944.2 \\
\hline Intemal Charges Fy 1995 : & & & & & 2 & 577.2 & & 17.3 & & 594.5 \\
\hline IRM Support FY 1995: & & & & & & 555.3 & $\therefore$ & 16.6 & ? & 671.9 \\
\hline Iotal Non-Labor & & 0.0 & & $\underline{0}$ & 12 & 2354.7 & & 70.4 & $\underline{0}$ & 2425.1 \\
\hline Total cost Account Within Iarget & 31.3 & 4918.0 & (19.3) & (1818.8) & 12 & 3099.2 & 0 & 92.6 & & 3191.8 \\
\hline
\end{tabular}




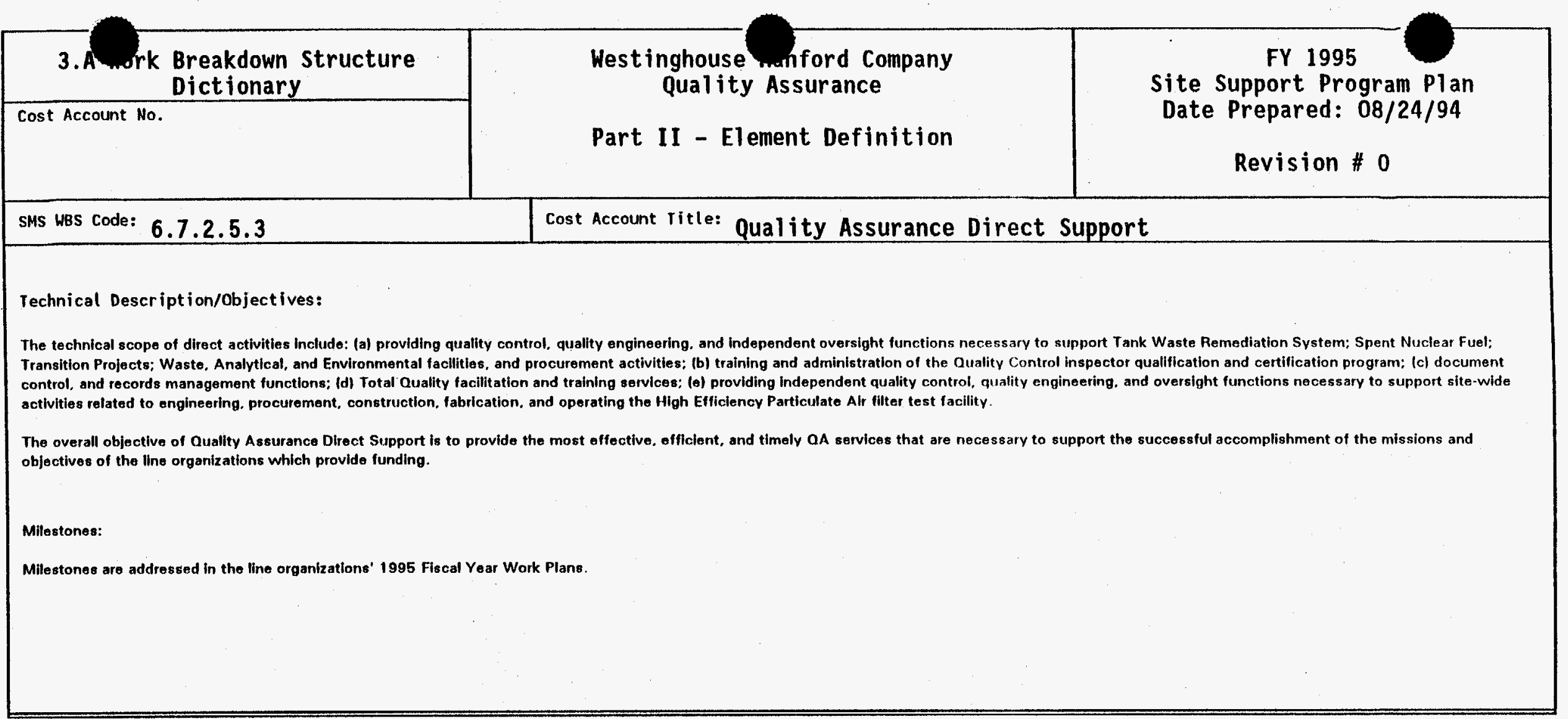


Activities include independent quality control, quality engineering, and oversight functions necessary to support the TWRS project facilities including the Tank Farms, 242-A Evaporator, Grout, HWVP, and other TWRS facilities.

WHC Labor Costs

Work Scope Detailed Descriprion

OA Standards and Administration Direct Support (WBS 6.7.2.5.3.2)

Activities includes quality control training support, documents contual dild tecords management, and QA training support.

Work Scope Detailed Description:

Spent Nuclear Fuel QA Direct Support (WBS 6.7.2.5.3.3)

Activities include independent quality control, quality engineering, and oversight functions necessary to support the Spent Nuclear Fuels project facilities including the K-Basins and other spent fuel activities.

WHC Labor Costs

Hork Scope Dẹtailed Description:

Iransition Projects QA Direct Suppont IWBS 6.7.2.5.3.41 Activities include independent quality control. quality engineering, and oversiglt functions necessary to support the
Transition Projects program facilities including PFP. FFTF, PUREX, B.Plant, and Fuel Fabrication Facilities Transition Projects.

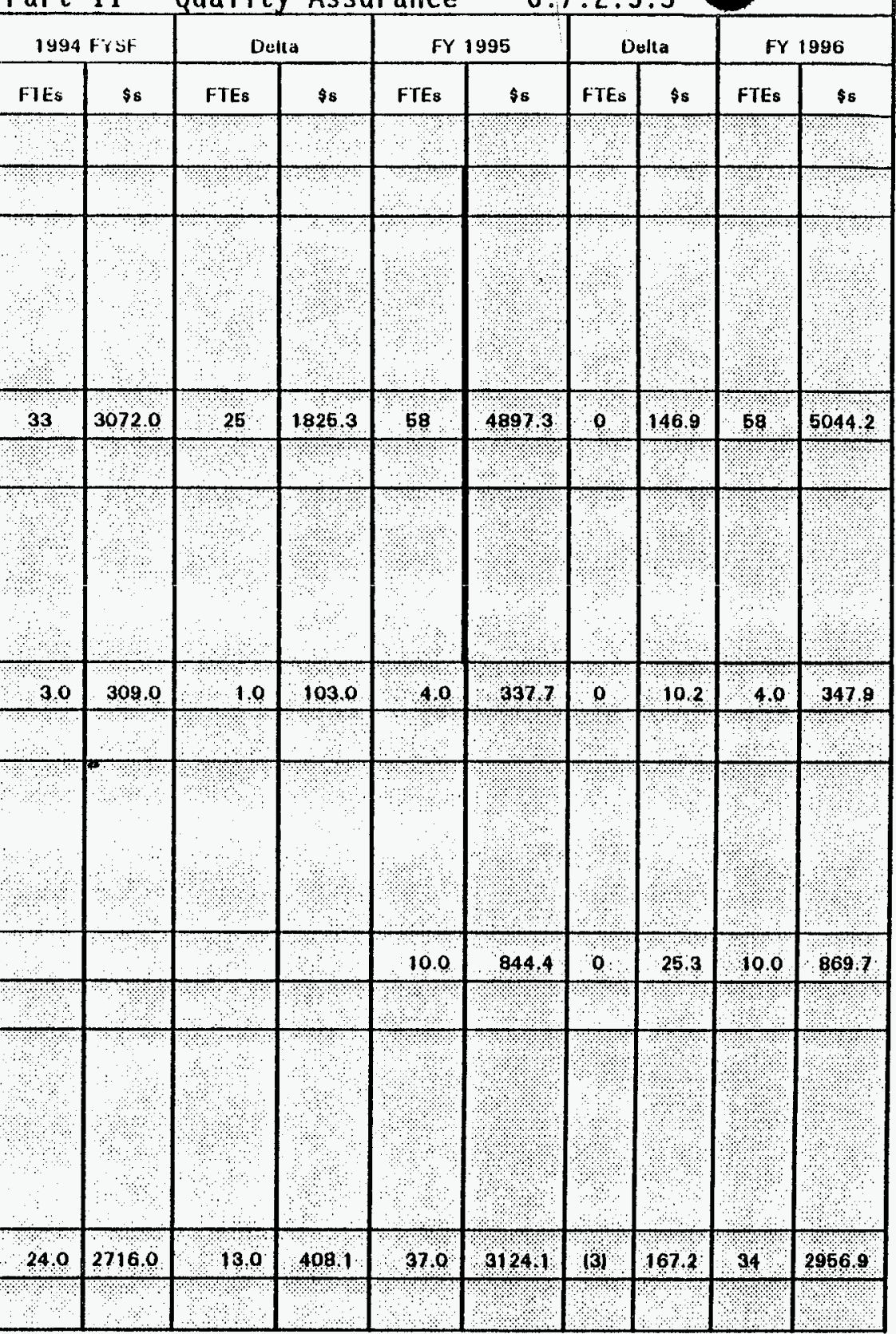




\begin{tabular}{|c|c|c|c|c|c|c|c|c|c|c|}
\hline Cost Acc, & Part & I - & uali & As & ance & -6.7 & .2 .5 & .3 & & \\
\hline $\begin{array}{l}\text { Element Task Description } \\
\text { Requests Within Target: }\end{array}$ & \multicolumn{2}{|c|}{1994 FYSF } & \multicolumn{2}{|c|}{ Delta } & \multicolumn{2}{|c|}{ FY 1995} & \multicolumn{2}{|c|}{ Delta } & \multicolumn{2}{|c|}{ FY 1996} \\
\hline \multirow{2}{*}{\multicolumn{11}{|c|}{$\begin{array}{l}\text { Work Scope Detailed Description: } \\
\text { Waste, Analytical and Environmental QA Direct Support (WBS 6.7.2.5.3.5) } \\
\text { Activities include independent quality control, quality engineering, and oversight functions necessary to support the } \\
\text { Waste, Analytical and Environmental program faeifities including the 222-S Analytical Laboratories, the Central Waste } \\
\text { Complex, T-Plant, and TRUSAF. }\end{array}$}} \\
\hline & & & & & & & & & & \\
\hline WHC Labor Costs & & & & & 440 & 37152 & 0 & 1114 & 44.0 & 3826.6 \\
\hline \multicolumn{11}{|l|}{$\begin{array}{l}\text { Work Scope Detai led Descript ion: } \\
\text { Projects and Procurement Direct Support (WBS-6.7.2.5.3.6) } \\
\text { Activities include independent quality control, quality engineering. and oversight functions necessary to support the } \\
\text { HEPA Filter Test Facility. Fabrication Shop, suppller evaluations, support for Stores, recoiving inspection, and } \\
\text { Construction Projects. }\end{array}$} \\
\hline WHC Labor Cost & & & & & 19.5 & 1646.5 & $\mathbf{0}$ & 49.4 & 19.5 & 1695.9 \\
\hline Total Labor & & & & & 172.5 & 14607.8 & (3) & 176.4 & 169.5 & 14741.2 \\
\hline Total Cost Account Wi thin Target & & & & & & & & & & \\
\hline
\end{tabular}

Notes: The FY 1994 and FY 1995 WBS and budget structures are completely different, making it impractical to compare them at any level below the cost account level.

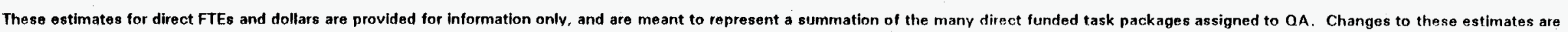
not subject to formal change control. 


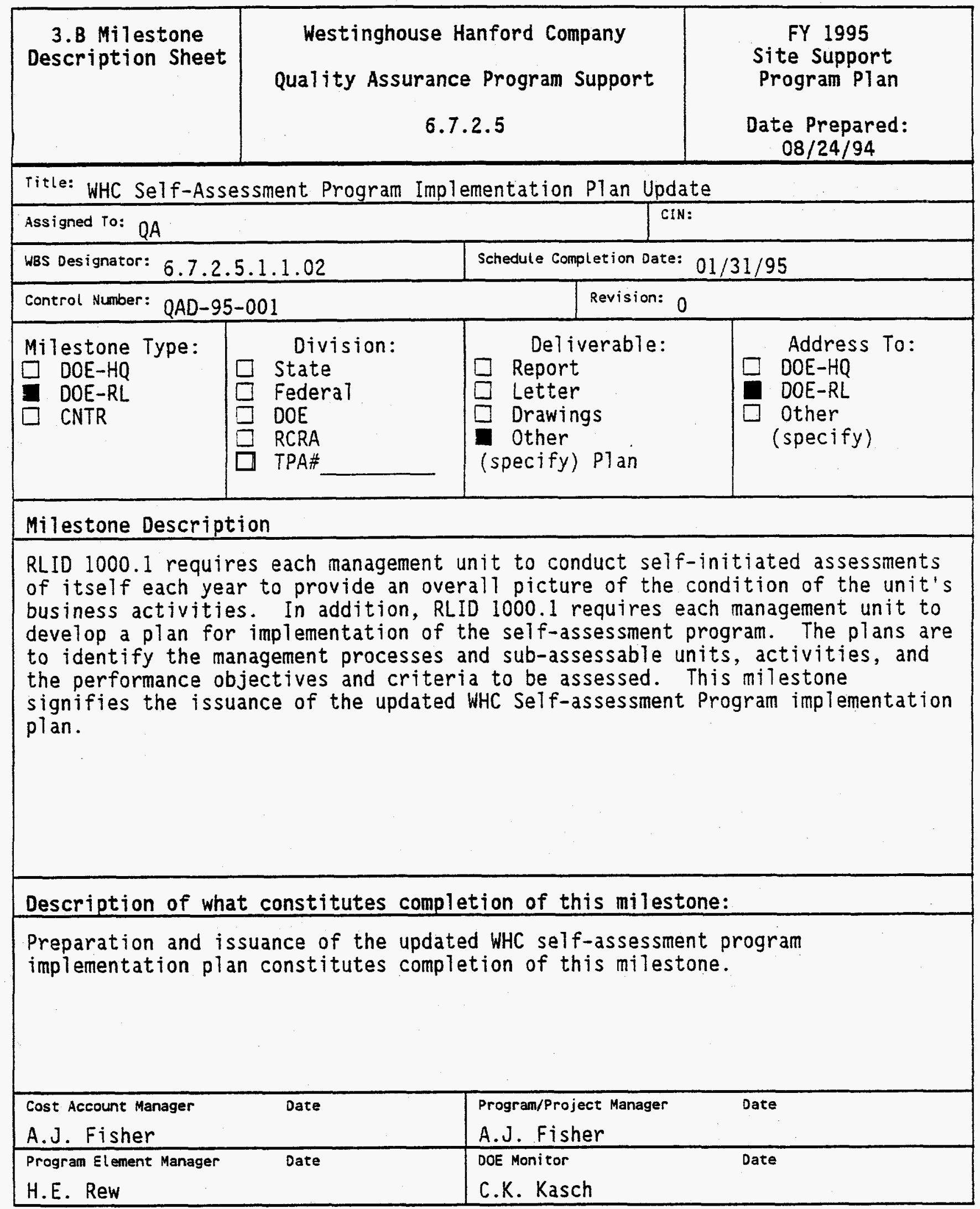




\begin{tabular}{|c|c|c|c|}
\hline $\begin{array}{c}\text { 3.B Milestone } \\
\text { Description Sheet }\end{array}$ & \multicolumn{2}{|c|}{$\begin{array}{c}\text { Westinghouse Hanford Company } \\
\text { Quality Assurance Program Support } \\
6.7 .2 .5\end{array}$} & $\begin{array}{c}\text { FY } 1995 \\
\text { Site Support } \\
\text { Program Plan } \\
\text { Date Prepared: } \\
\quad 08 / 24 / 94\end{array}$ \\
\hline \multicolumn{4}{|c|}{ Title: Mid-year WHC Self-Assessment Report } \\
\hline \multicolumn{3}{|c|}{ Assigned To: $Q A$} & \\
\hline \multicolumn{3}{|c|}{ WBS Designator: 6.7 .2 .5 .1 .1 .02} & $15 / 95$ \\
\hline \multicolumn{3}{|c|}{ Control Number: $Q A D-95-002$} & \\
\hline $\begin{array}{l}\text { Milestone Type: } \\
\square \text { DOE-HQ } \\
\text { DOE-RL } \\
\square \text { CNTR }\end{array}$ & \begin{tabular}{ll} 
& \multicolumn{1}{c}{ Di } \\
$\square$ & Stat \\
$\square$ & Fede \\
$\square$ & DOE \\
$\square$ & RCRA \\
$\square$ & TPA
\end{tabular} & $\begin{array}{l}\text { Deliverable } \\
\text { Report } \\
\square \text { Letter } \\
\square \text { Drawings } \\
\square \text { Other } \\
\text { (specify) }\end{array}$ & $\begin{array}{l}\text { Address To: } \\
\square \text { DOE-HQ } \\
\text { DOE-RL } \\
\square \text { Other } \\
\text { (specify) }\end{array}$ \\
\hline \multicolumn{4}{|c|}{ Milestane Description } \\
\hline \multicolumn{4}{|c|}{$\begin{array}{l}\text { RLID } 1000.1 \text { requires each management unit to report annually on the conduct of } \\
\text { self-initiated assessments of itself to provide an overall picture of the } \\
\text { condition of the unit's business activities. In addition, RLID } 1000.1 \text { requires } \\
\text { each management unit to issue a status report six months after the annual } \\
\text { report. This report is to identify any changes in performance objectives or the } \\
\text { self-assessment process and the status of key corrective actions. This } \\
\text { milestone signifies the issuance of the mid-year status report on the wHC roll } \\
\text { up consolidation of individual units' self-assessment results. }\end{array}$} \\
\hline \multicolumn{4}{|c|}{ Description of what constitutes completion of this milestone: } \\
\hline \multicolumn{4}{|c|}{$\begin{array}{l}\text { Preparation and issuance of the mid-year status report on the WHC roll up } \\
\text { consolidation of individual units' self-assessment results constitutes } \\
\text { completion of this milestone. }\end{array}$} \\
\hline $\begin{array}{l}\text { Cost Account Manager } \\
\text { A.J. Fisher }\end{array}$ & Date & $\begin{array}{l}\text { Program/Project Manager } \\
\text { A.J. Fisher }\end{array}$ & Date \\
\hline \multicolumn{2}{|l|}{$\begin{array}{l}\text { Program Element Manager } \\
\text { H.E. Rew }\end{array}$} & $\begin{array}{l}\text { DoE Monitor } \\
\text { C.K. Kasch }\end{array}$ & Date \\
\hline
\end{tabular}




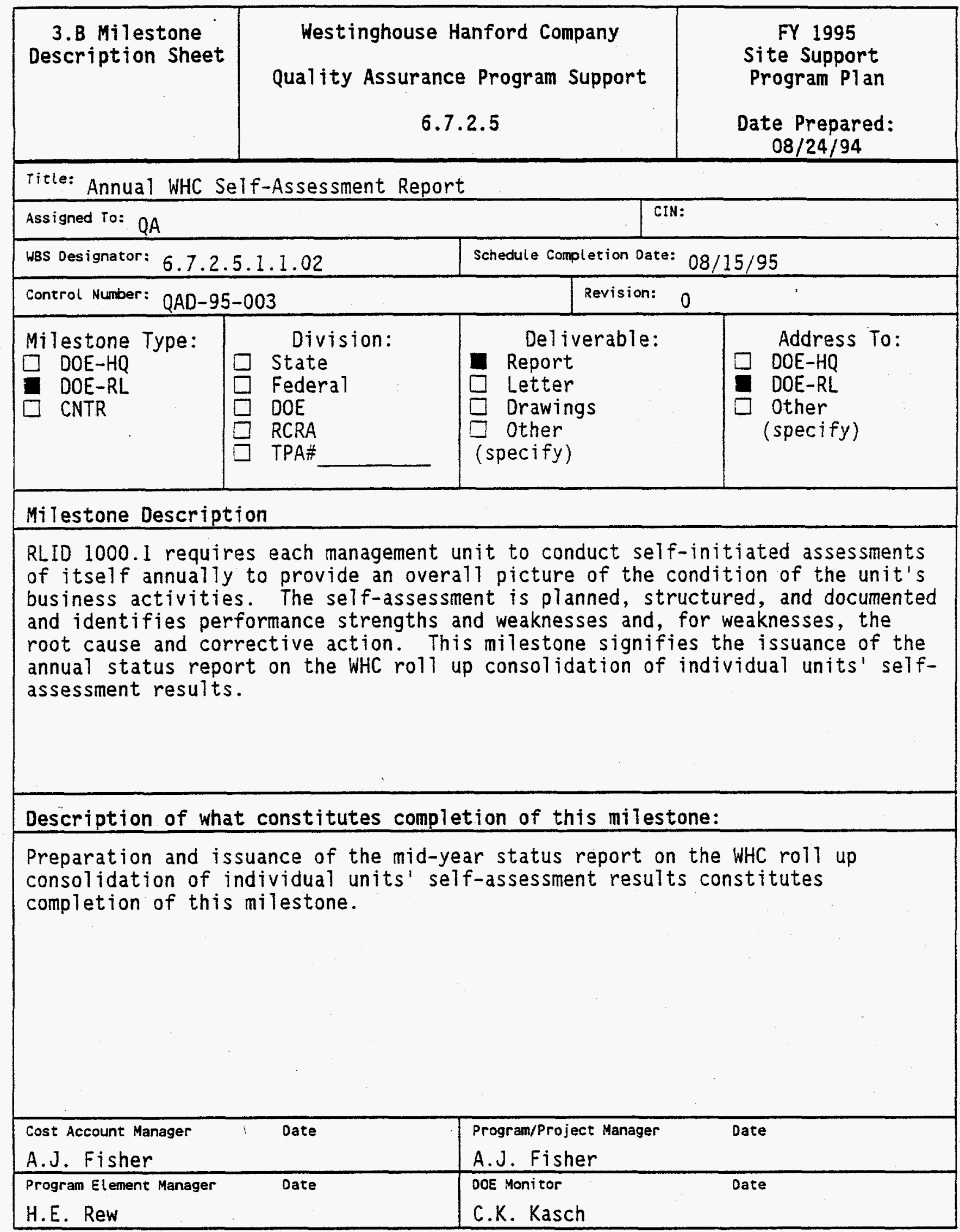




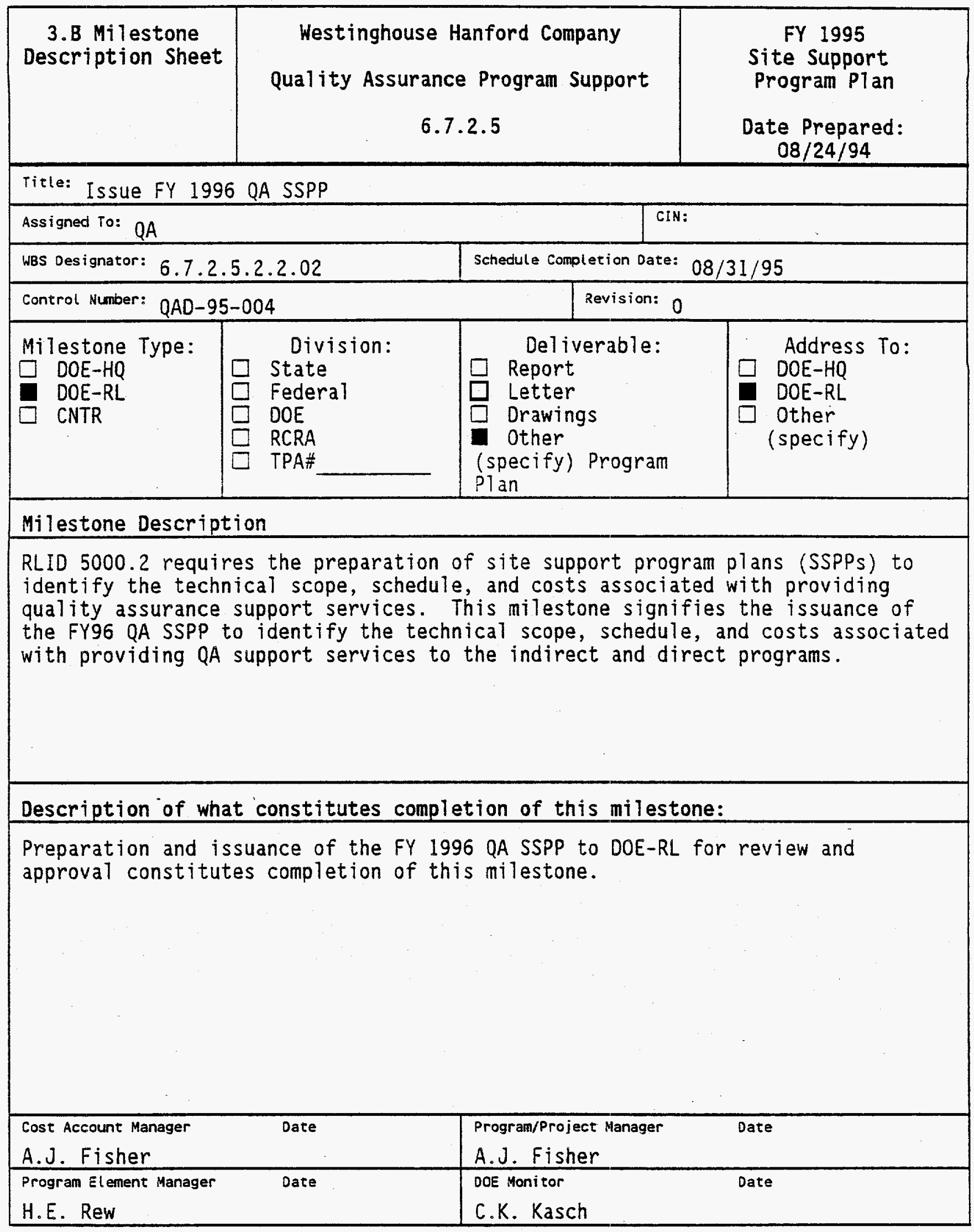




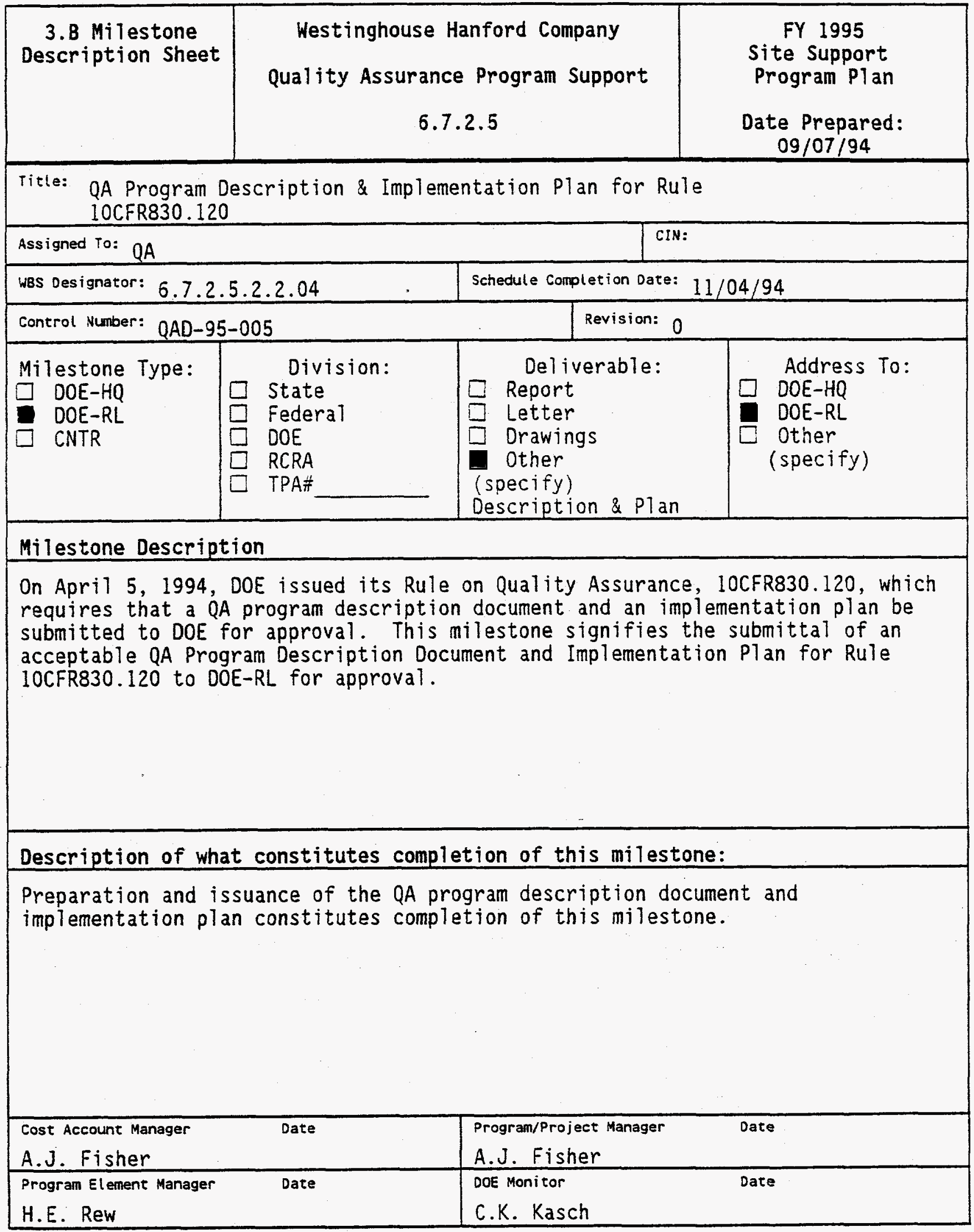




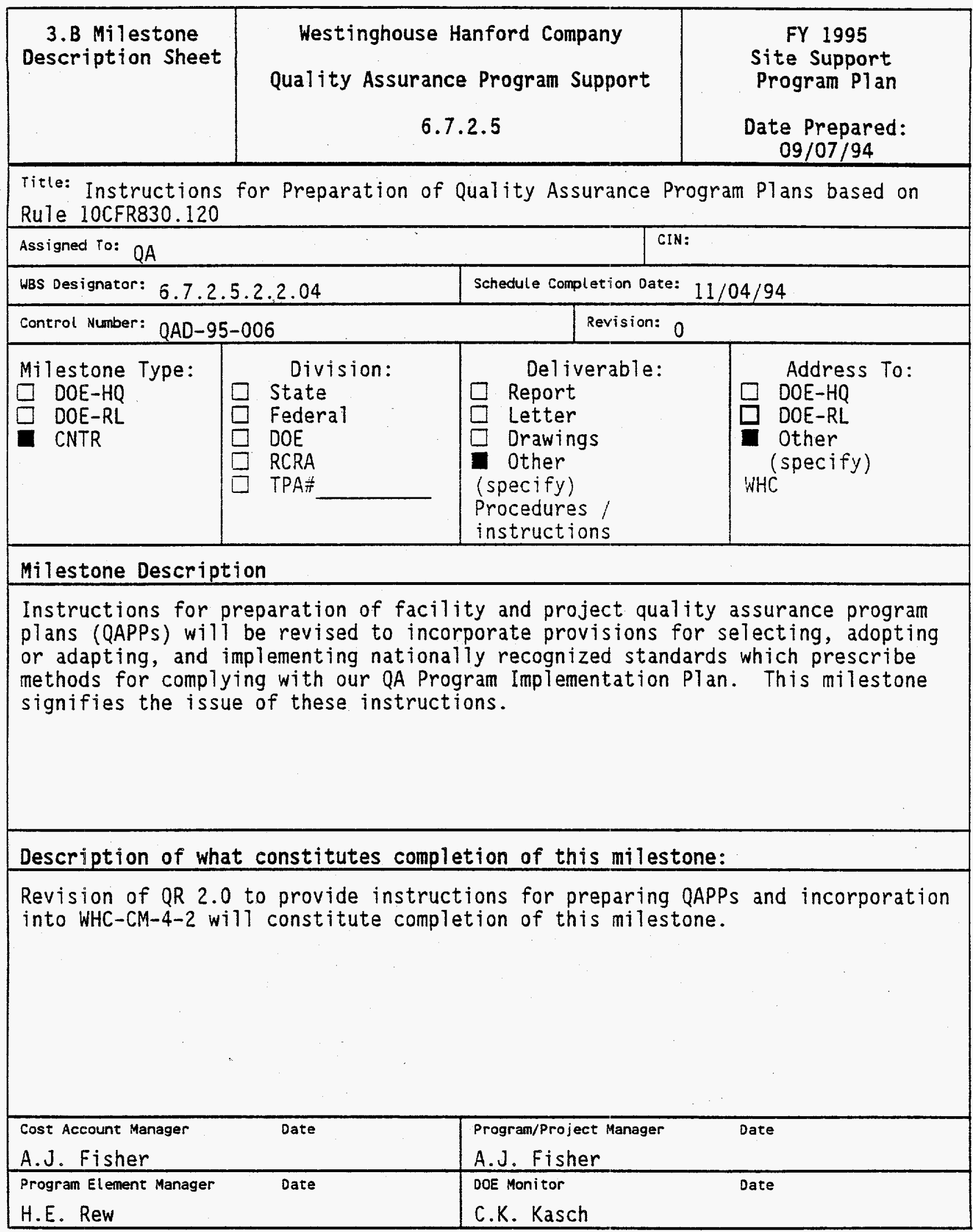




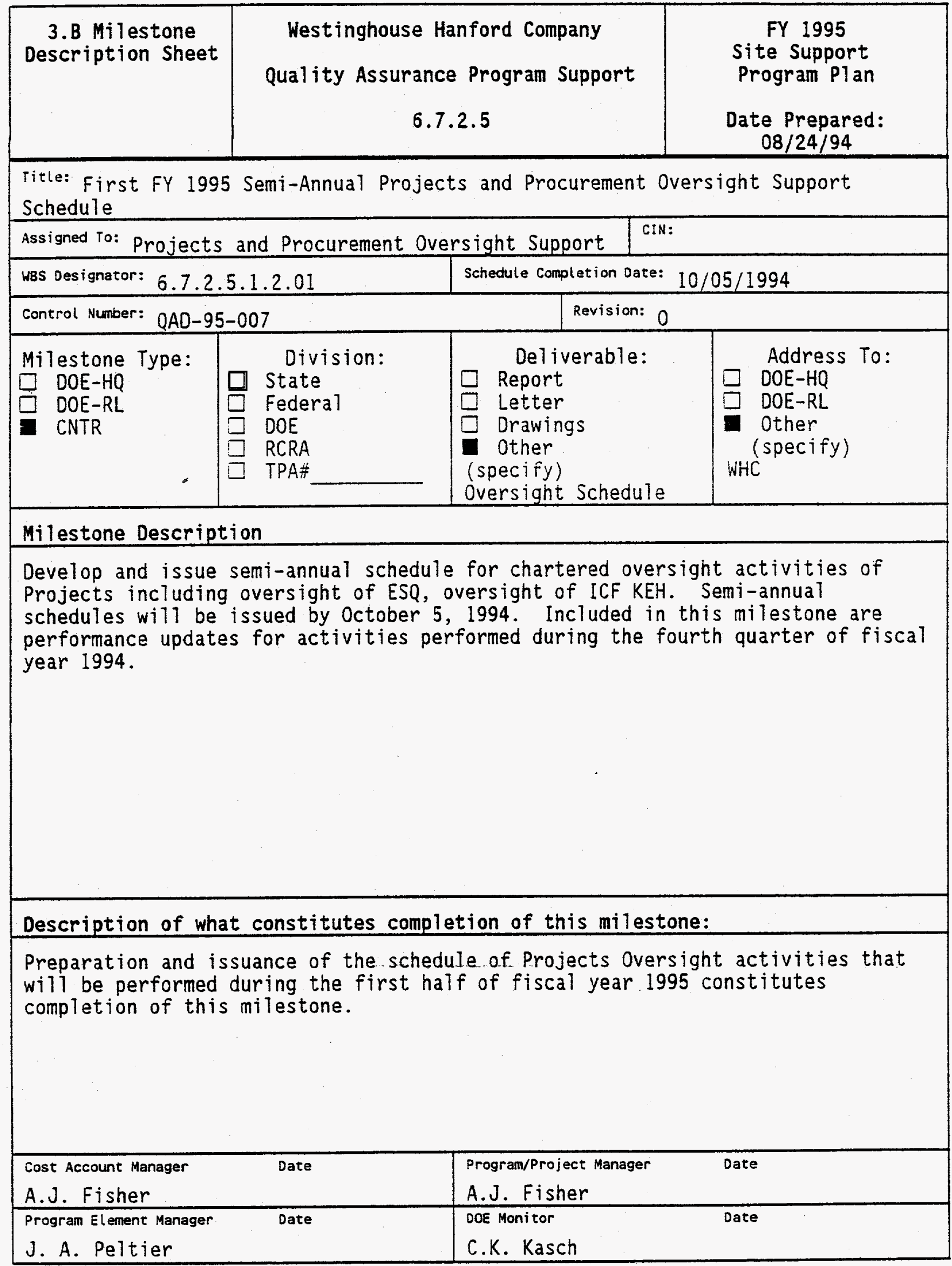




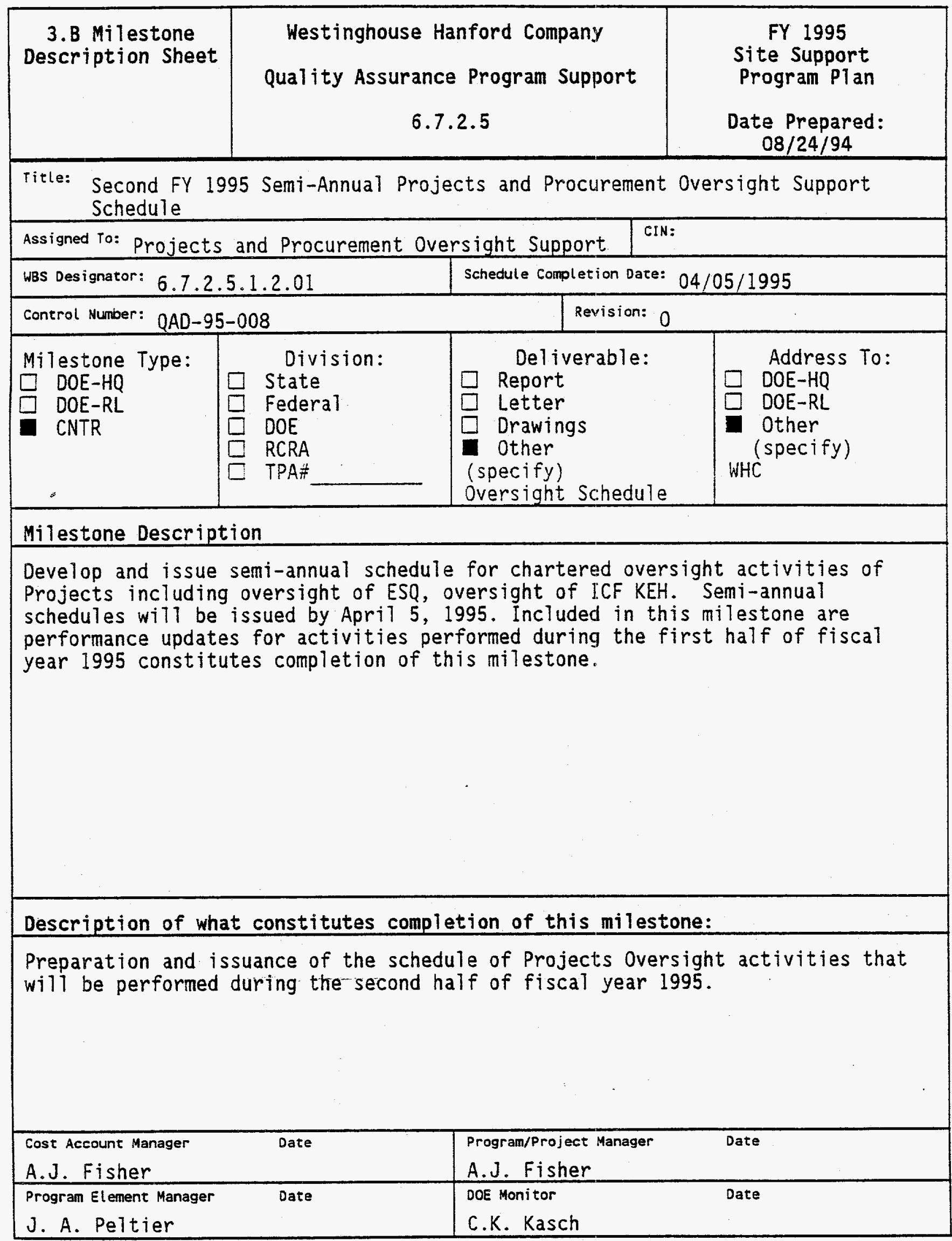




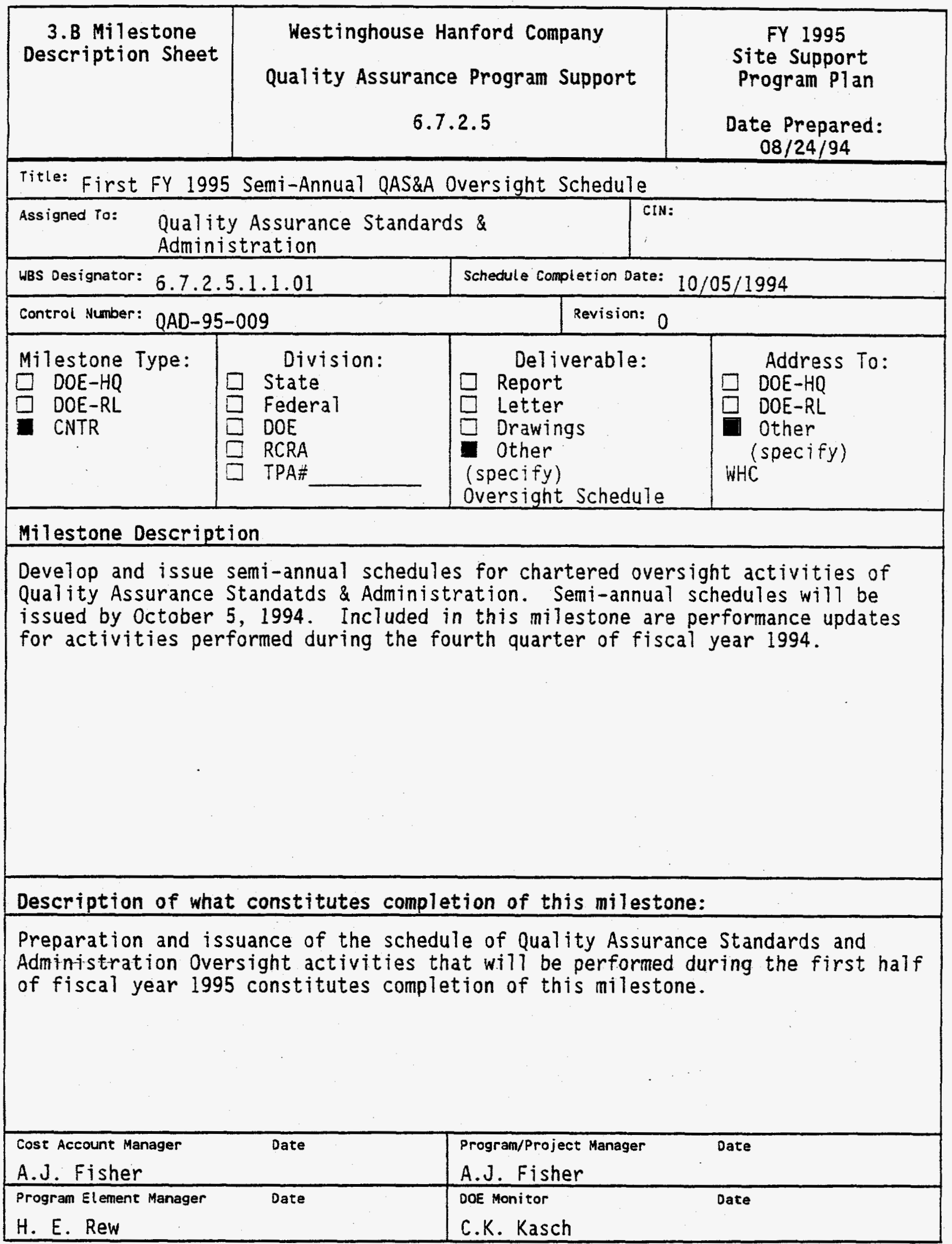




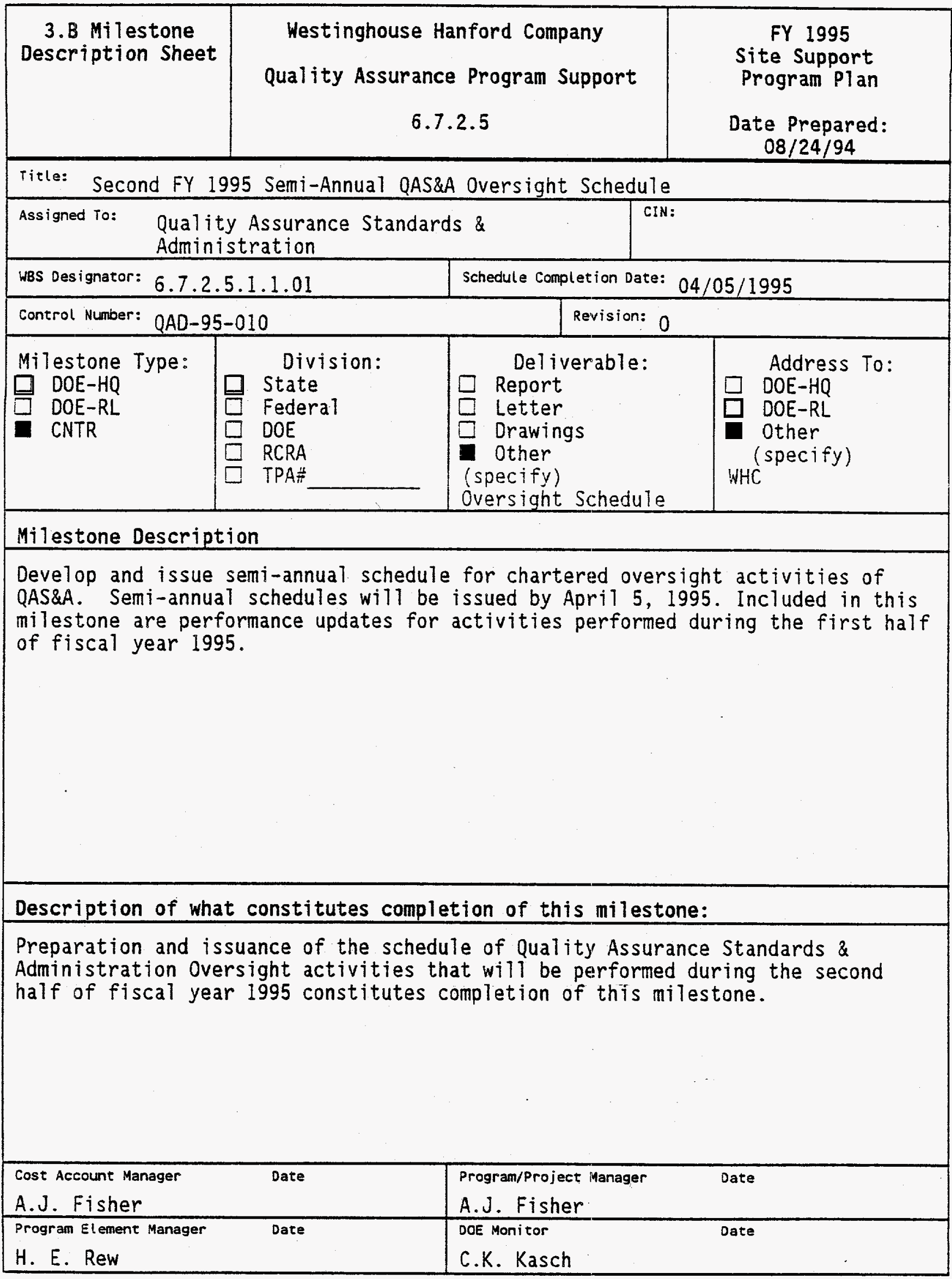




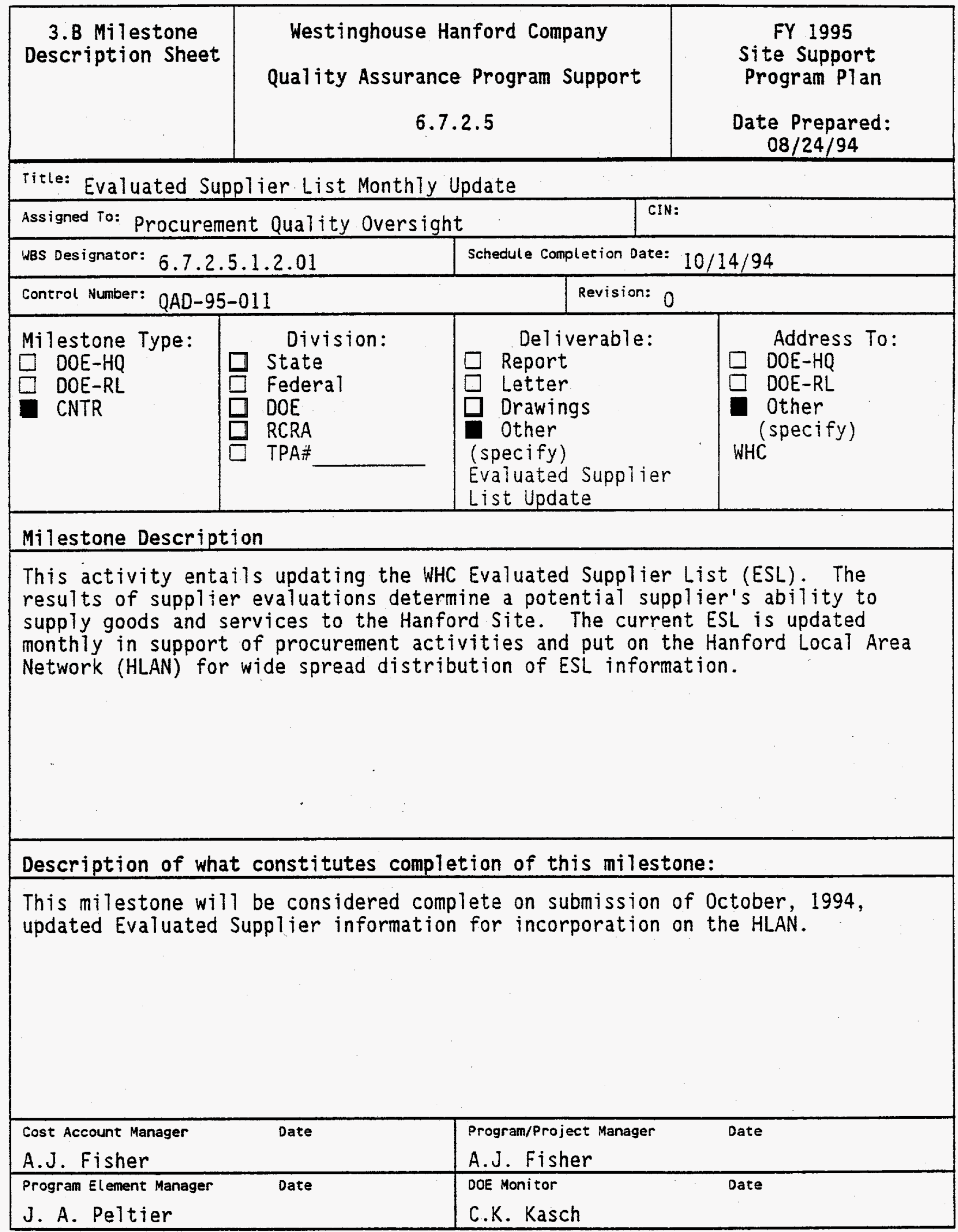




\begin{tabular}{|c|c|c|c|}
\hline $\begin{array}{l}\text { 3.B Milestone } \\
\text { Description Sheet }\end{array}$ & \multicolumn{2}{|c|}{$\begin{array}{l}\text { Westinghouse Hanford Company } \\
\text { Quality Assurance Program Support } \\
\qquad 6.7 .2 .5\end{array}$} & $\begin{array}{l}\text { Fy } 1995 \\
\text { Site Support } \\
\text { Program Plan } \\
\text { Date Prepared: } \\
\quad 08 / 24 / 94\end{array}$ \\
\hline \multicolumn{4}{|c|}{ Title: Evaluated Supplier List Monthly Update } \\
\hline \multicolumn{3}{|c|}{ Assigned To: Procurement Quality Oversight } & \\
\hline \multicolumn{2}{|c|}{ WBS Designator: 6.7 .2 .5 .1 .2 .01} & \multicolumn{2}{|c|}{ Schedule Completion Date: $11 / 15 / 94$} \\
\hline \multicolumn{2}{|c|}{ Control Number: $Q A D-95-012$} & Revision: & \\
\hline $\begin{array}{l}\text { Milestone Type: } \\
\square \text { DOE-HQ } \\
\square \text { DOE-RL } \\
\square \text { CNTR }\end{array}$ & \begin{tabular}{ll} 
& \multicolumn{1}{c}{ Division: } \\
$\square$ & State \\
$\square$ & Federal \\
$\square$ & DOE \\
$\square$ & RCRA \\
$\square$ & TPA\# \\
\end{tabular} & $\begin{array}{l}\text { Deliverable: } \\
\square \text { Report } \\
\square \text { Letter } \\
\square \text { Orawings } \\
\text { Other } \\
\text { (specify) } \\
\text { Evaluated Supplier } \\
\text { List Update }\end{array}$ & $\begin{array}{l}\text { Address To: } \\
\square \text { DOE-HQ } \\
\square \text { DOE-RL } \\
\text { Other } \\
\text { (specify) } \\
\text { WHC }\end{array}$ \\
\hline \multicolumn{4}{|c|}{ Milestone Description } \\
\hline \multicolumn{4}{|c|}{$\begin{array}{l}\text { This activity entails updating the WHC Evaluated Supplier List (ESL). The } \\
\text { results of supplier evaluations determine a potential supplier's ability to } \\
\text { supply goods and services to the Hanford Site. The current ESL is updated } \\
\text { monthly in support of procurement activities and put on the Hanford Local Area } \\
\text { Network (HLAN) for wide spread distribution of ESL information. }\end{array}$} \\
\hline \multicolumn{4}{|c|}{ Description of what constitutes completion of this milestone: } \\
\hline \multicolumn{4}{|c|}{$\begin{array}{l}\text { This milestone will be considered complete on submission of November, } 1994 \text {, } \\
\text { updated Evaluated Supplier information for incorporation on the HLAN. }\end{array}$} \\
\hline \multicolumn{2}{|c|}{$\begin{array}{l}\text { Cost Account Manager Date } \\
\text { A.J. Fisher }\end{array}$} & $\begin{array}{l}\text { Program/Project Manager } \\
\text { A.J. Fisher }\end{array}$ & Date \\
\hline \multicolumn{2}{|c|}{$\begin{array}{l}\text { Program Element Manager Date } \\
\text { J. A. Peltier }\end{array}$} & $\begin{array}{l}\text { DOE Monitor } \\
\text { C.K. Kasch }\end{array}$ & Date \\
\hline
\end{tabular}




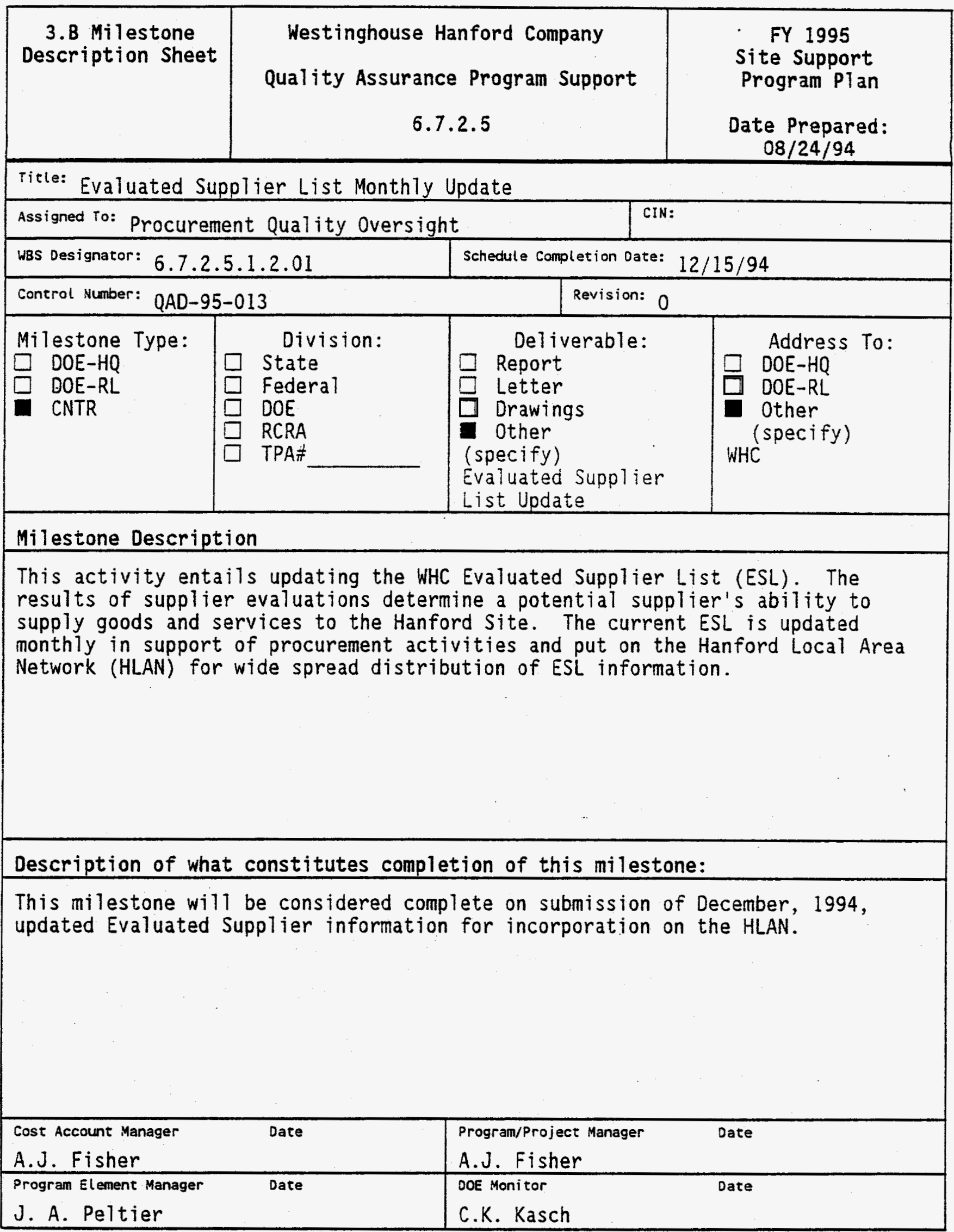




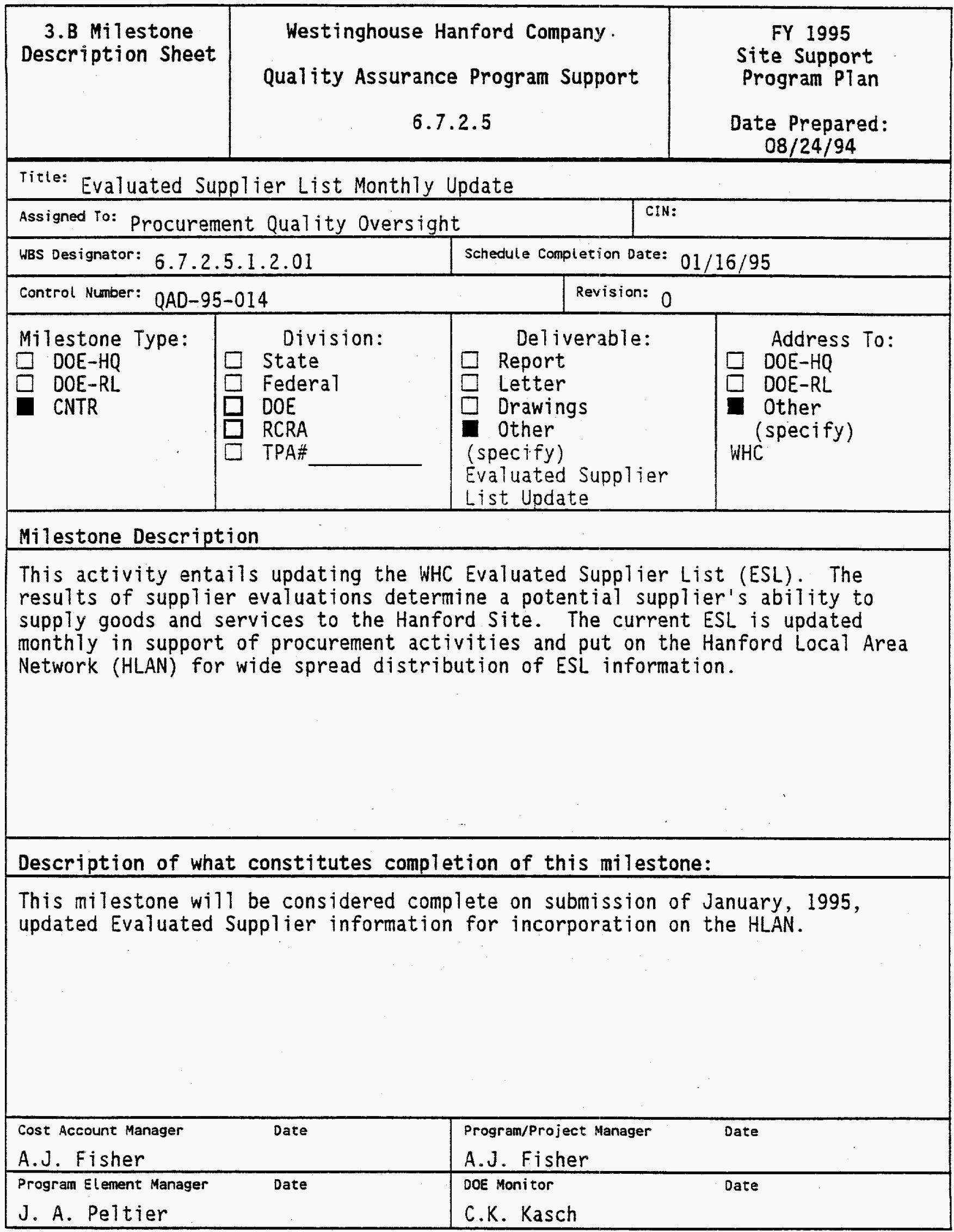




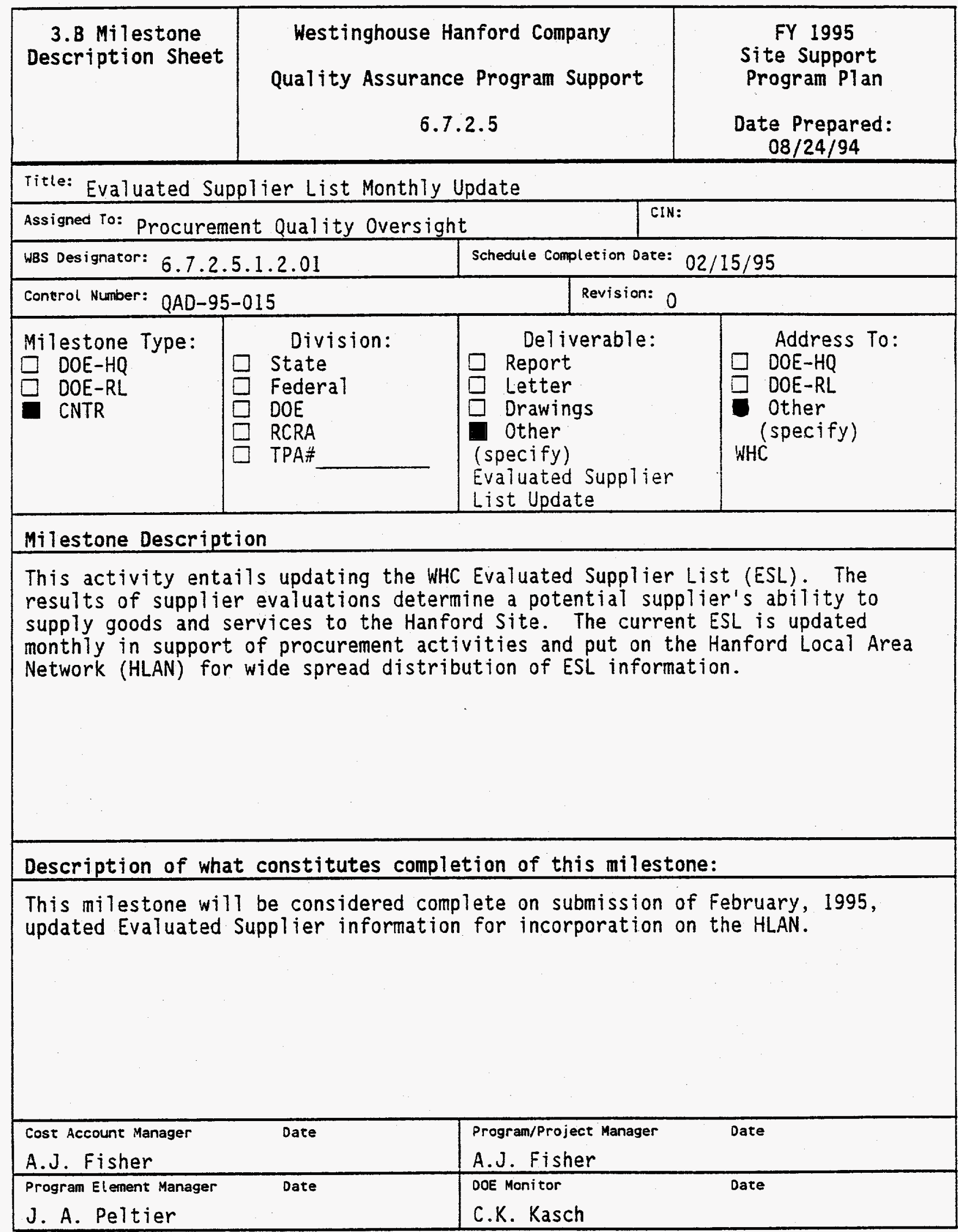




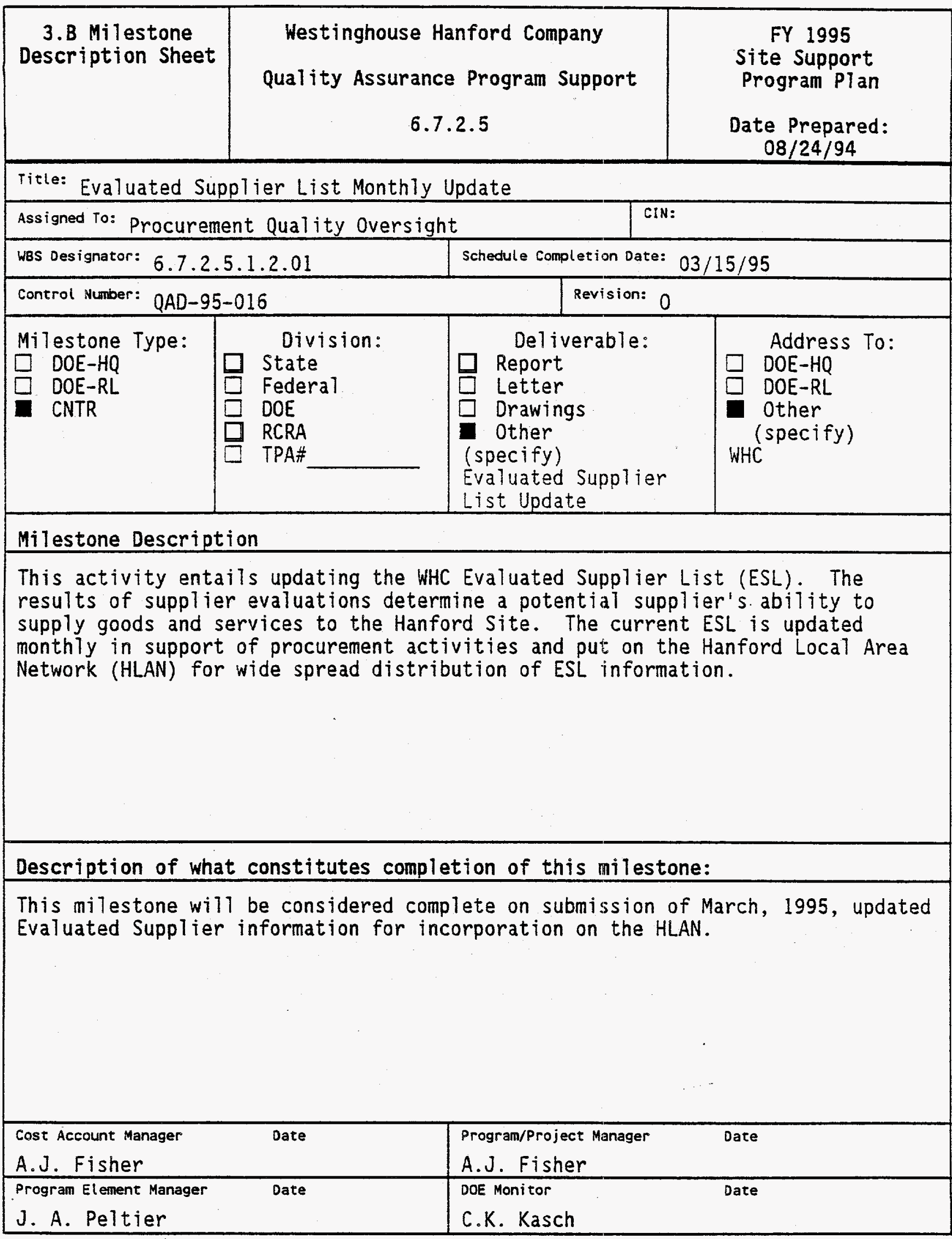




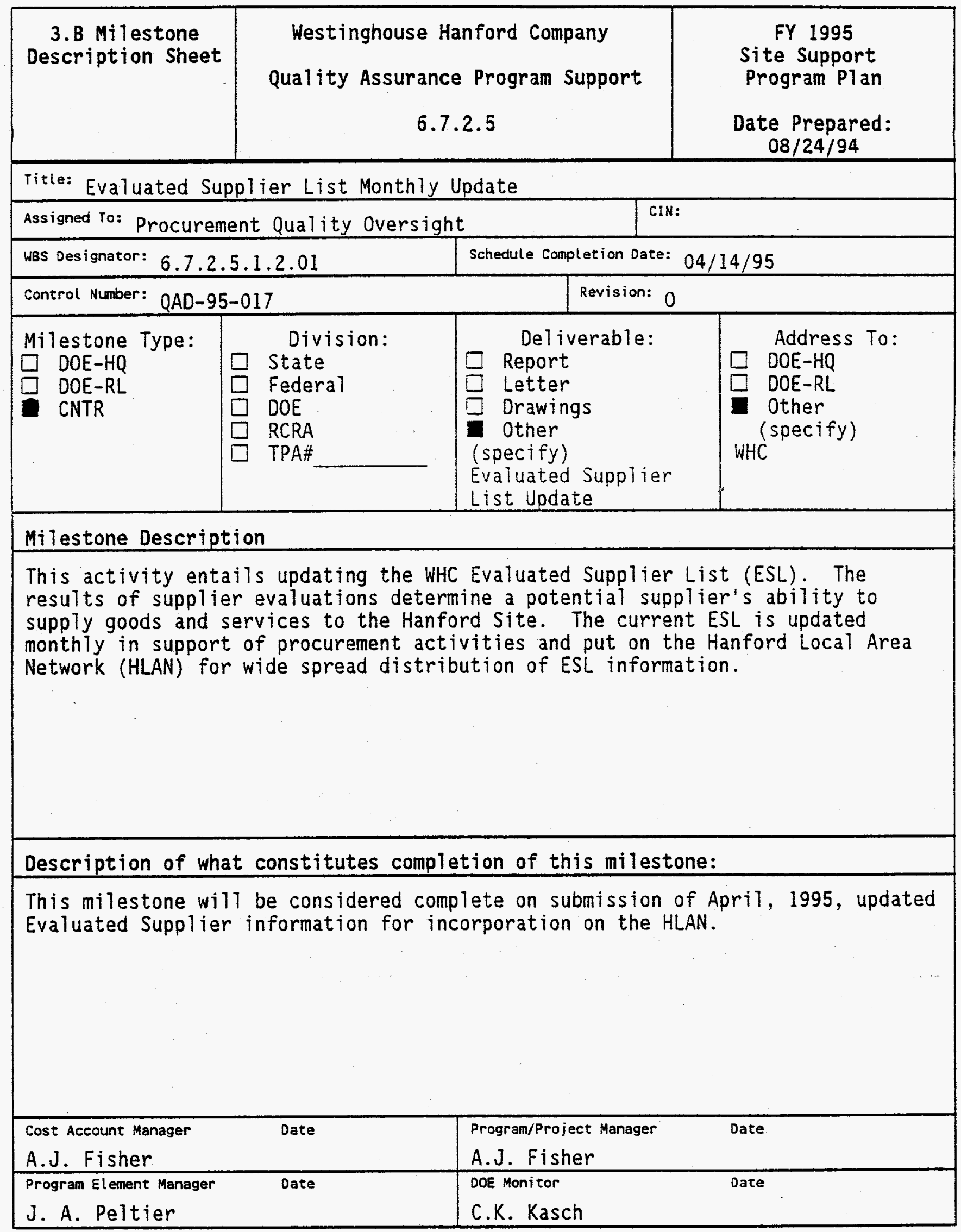




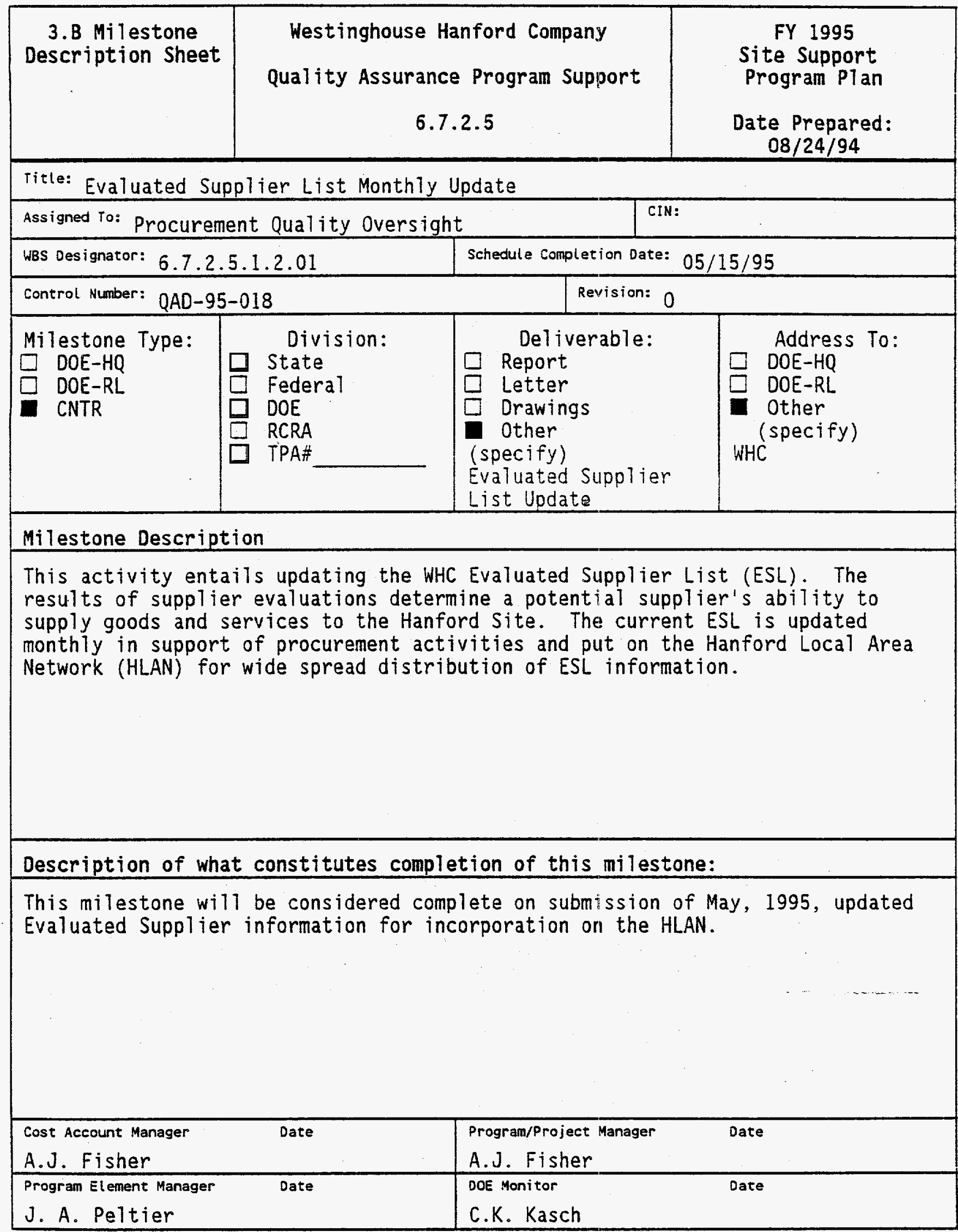




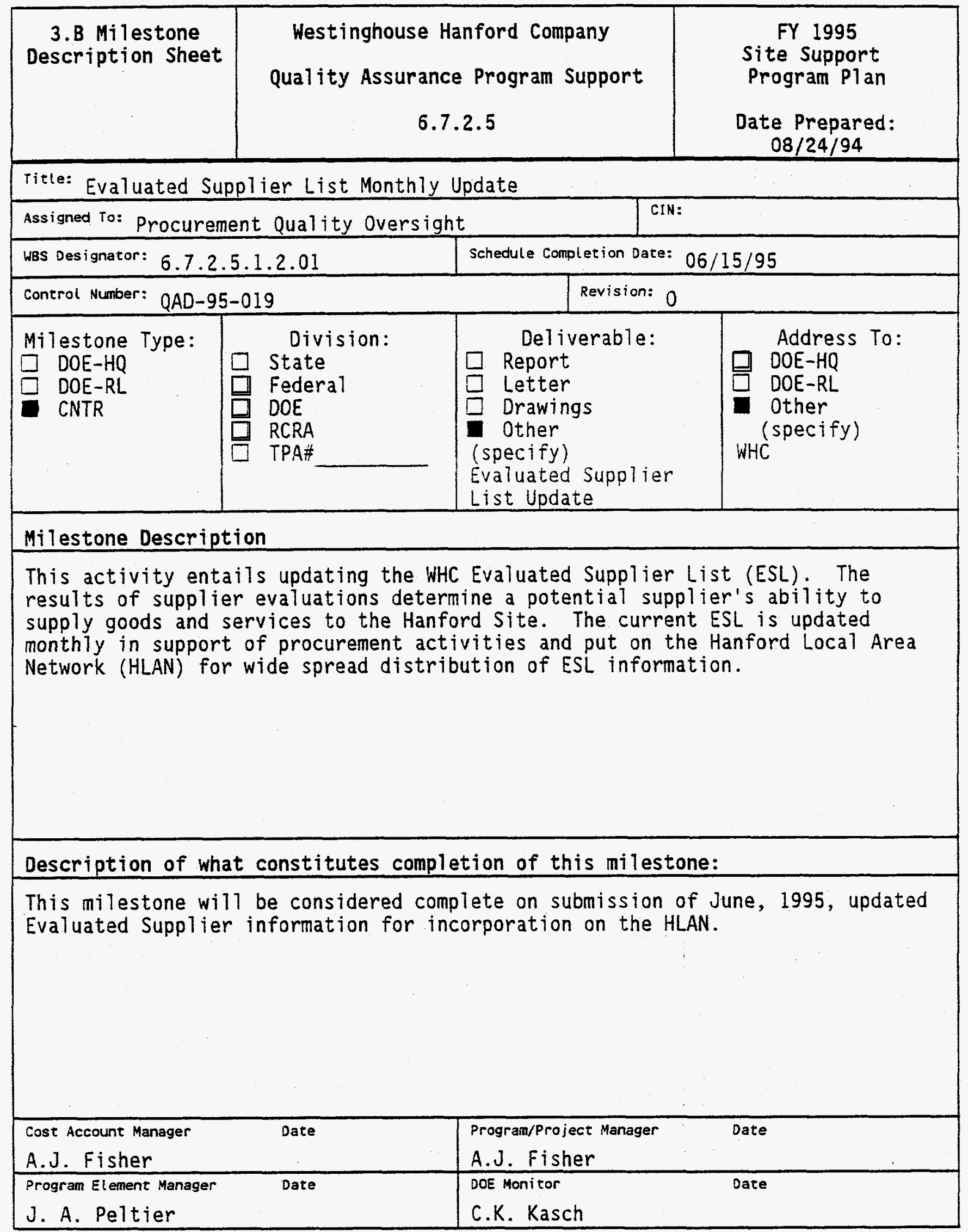




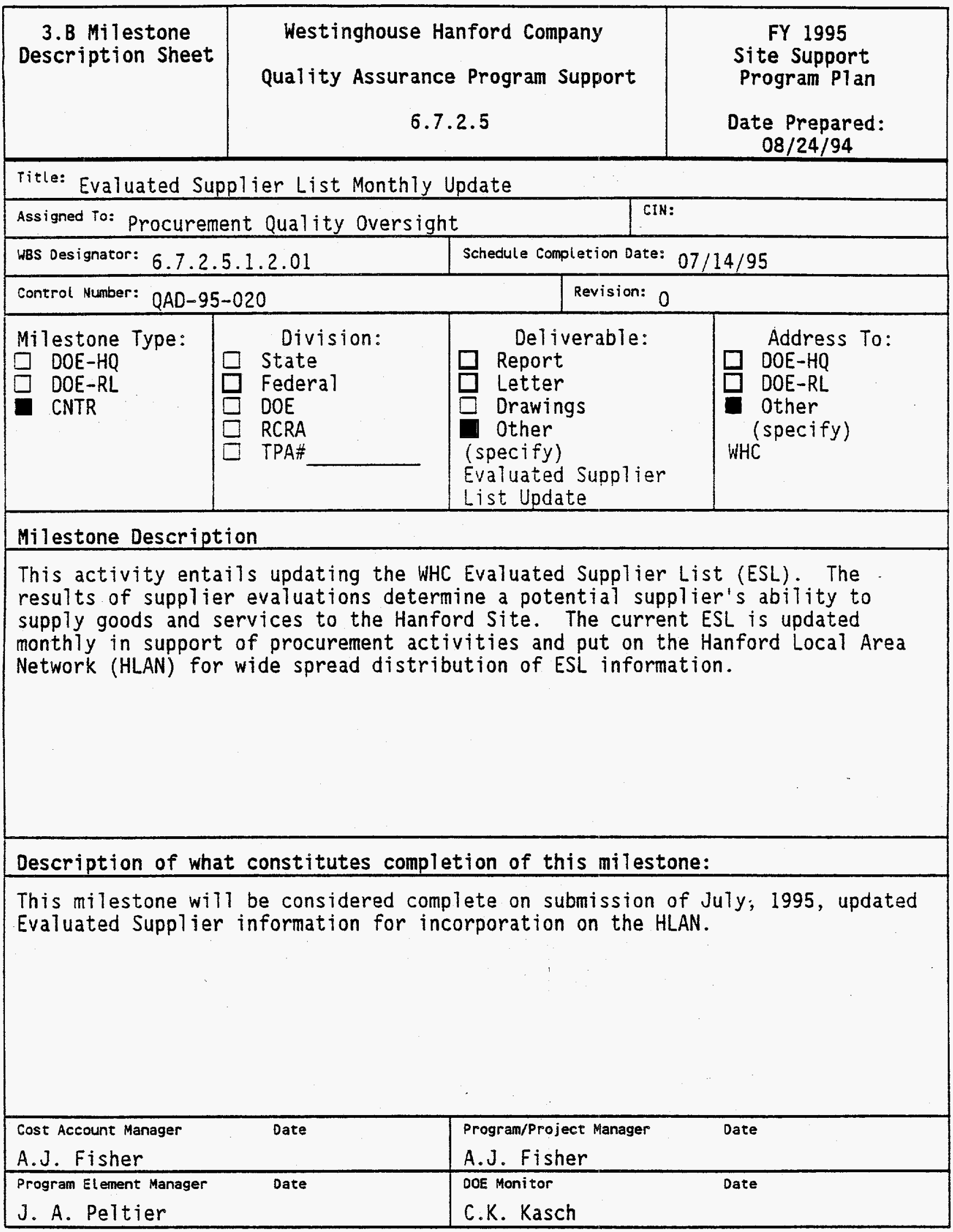




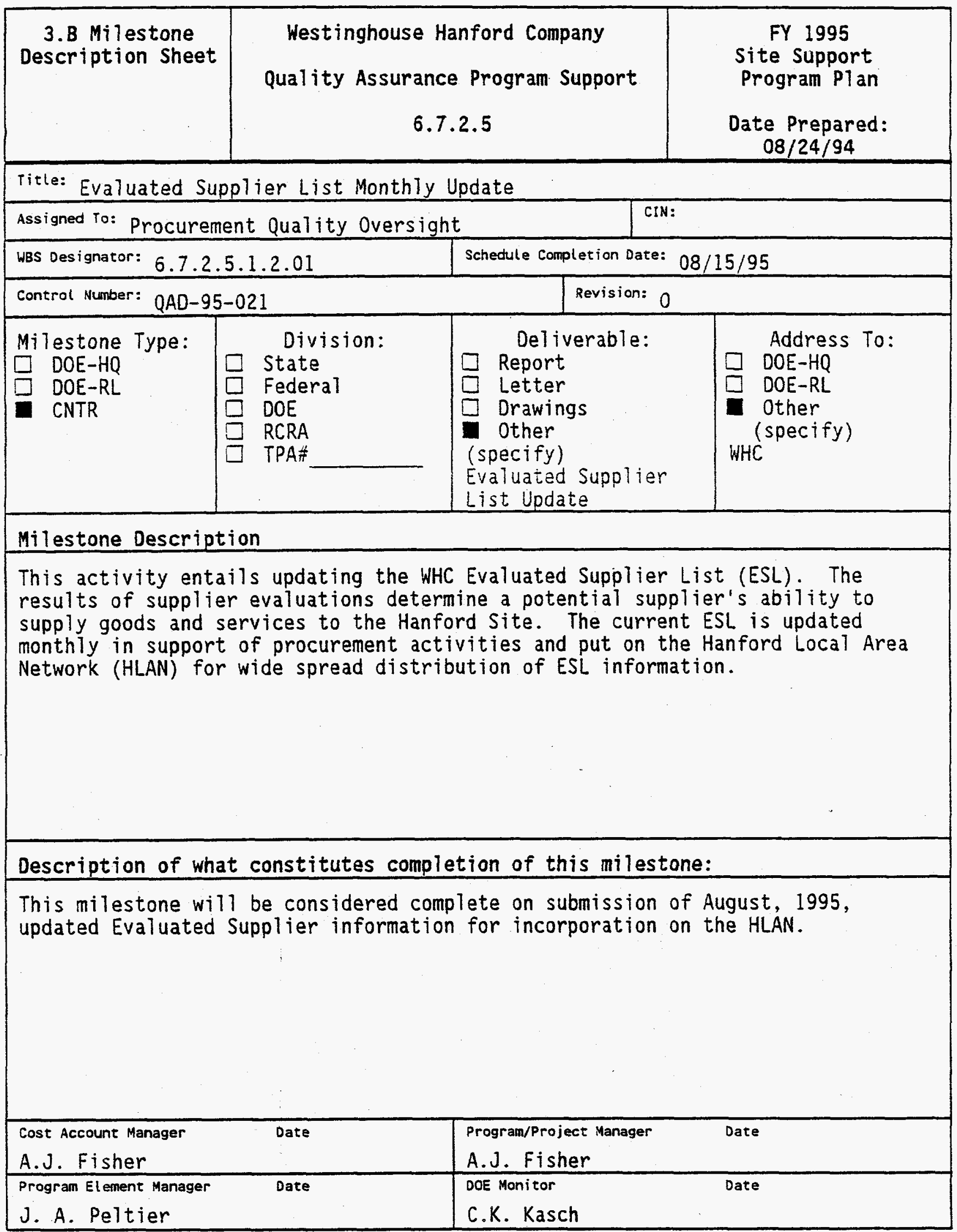




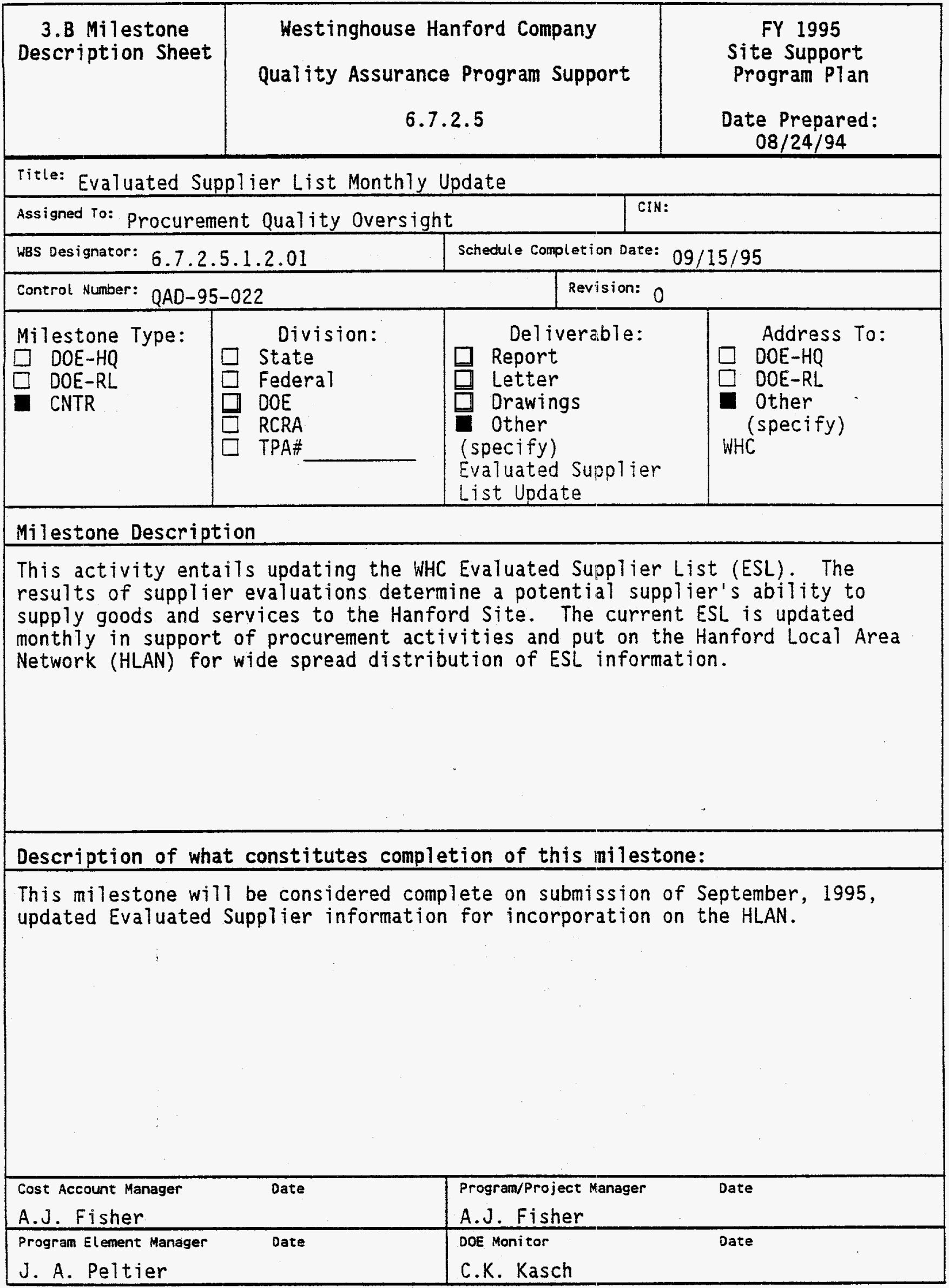




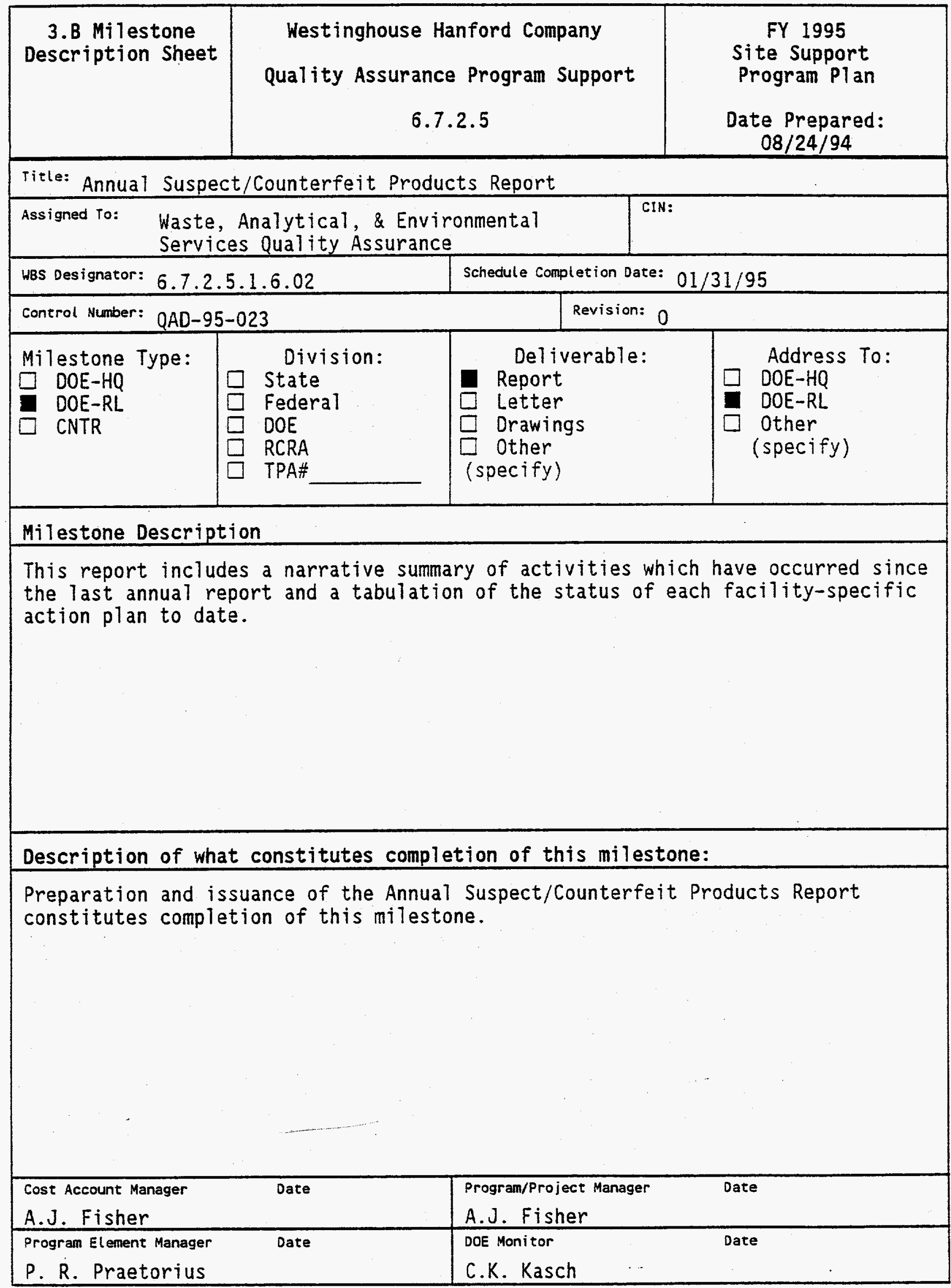

